\title{
Insights into the Enhanced in vivo Fitness of Neisseria gonorrhoeae Driven by a Fluoroquinolone Resistance-Conferring Mutant DNA Gyrase
}

\author{
by
}

Jonathan Andrew D’Ambrozio

Dissertation submitted to the Faculty of the Molecular and Cell Biology Graduate Program Uniformed Services University of the Health Sciences In partial fulfillment of the requirements for the degree of Doctor of Philosophy Molecular and Cell Biology 2015 
Name of Student: MAJ Jonathan D'Ambrozio

Date of Examination: November 12, 2014

Time: $1: 30 \mathrm{pm}$

Place: C2095

DECISION OF EXAMINATION COMMITTEE MEMBERS:
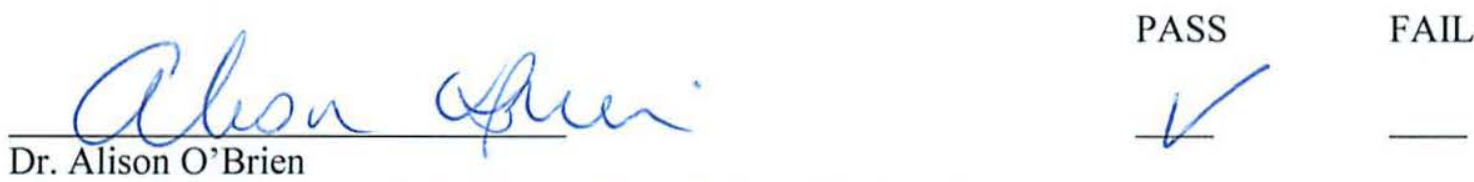

DEPARTMENT OF MICROBIOLOGY AND IMMUNOLOGY

Committee Chairperson

Dr. Ann Jerse

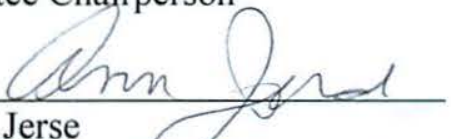

DEPARTMENT OF MICROBIOLOGY AND IMMUNOLOGY

Dissertation Advisor

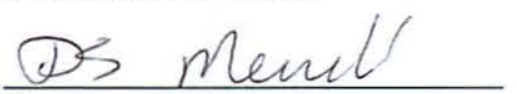

Dr. Douglas Merrell

DEPARTMENT OF MICROBIOLOGY AND IMMUNOLOGY

Committee Member

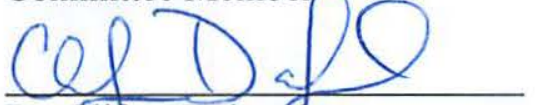

Dr. Clifton Dalgard

DEPARTMENT OF ANATOMY, PHYSIOLOGY, AND GENETICS

Committee Member

willen the

Dr. William Shafer

DEPARTMENT OF MICROBIOLOGY

EMORY UNIVERSITY

Committee Member
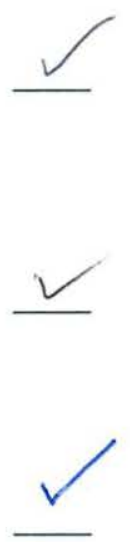

AIL 
APPROVAL OF THE DOCTORAL DISSERTATION IN THE MOLECULAR AND CELL BIOLOGY GRADUATE PROGRAM

Title of Dissertation: "Insights into the Enhanced in vivo Fitness of Neisseria gonorrhoeae Driven by a Fluoroquinolone Resistance-Conferring Mutant DNA Gyrase"

Name of Candidate: MAJ Jonathan D'Ambrozio

Doctor of Philosophy Degree

November 12, 2014

DISSERTATION AND ABSTRACT APPROVED:

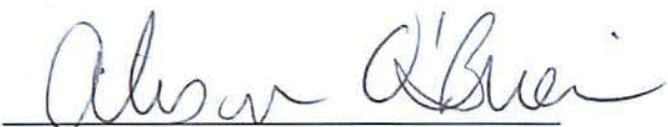

Dr. Alison O'Brien

DEPARTMENT OF MICROBIOLOGY AND IMMUNOLOGY

Committee Chairperson

Dr. Ann Jerse
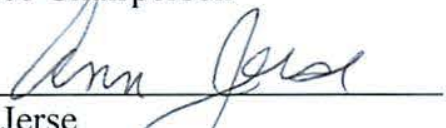

DEPARTMENT OF MICROBIOLOGY AND IMMUNOLOGY

Dissertation Advisor

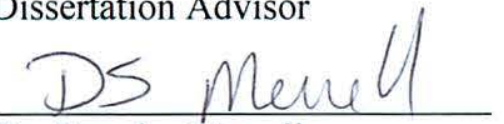

Dr. Douglas Merrell

DEPARTMENT OF MICROBIOLOGY AND IMMUNOLOGY

Committee Member
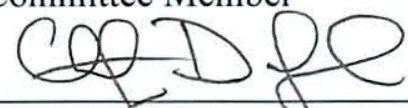

Dr. Cliftor Dalgard

DEPARTMENT OF ANATOMY, PHYSIOLOGY, AND GENETICS

Committee Member

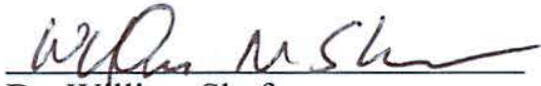

Dr. William Shafer

DEPARTMENT OF MICROBIOLOGY

EMORY UNIVERSITY

Committee Member
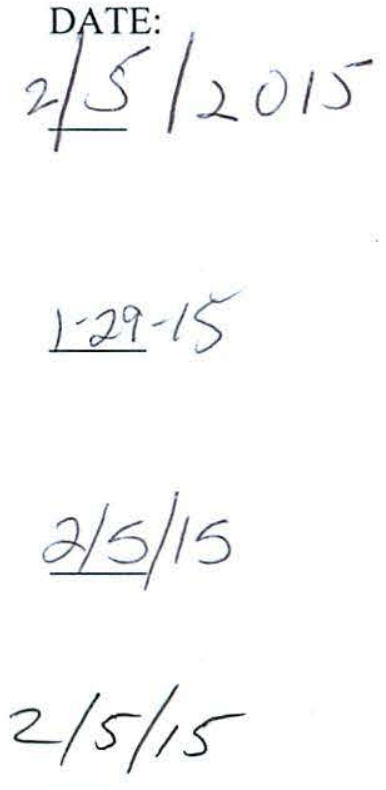

$11 / / 2 / 2014$ 


\section{ACKNOWLEDGMENTS}

I wish to acknowledge all of the individuals who facilitated the completion of this effort. I would like to thank Lee Metcalf, PhD, and Mary Lou Cutler, PhD, for welcoming me to the MCB program at USUHS. Ann Jerse, $\mathrm{PhD}$ welcomed me into her lab and graciously pulled me into a fascinating project that allowed me to immediately build upon my skill set; I have her to thank for her untiring mentorship, patience, and encouragement. All the members of the Jerse lab, past and present, provided invaluable suggestions and support throughout numerous lab meetings and experimental assistance;

these are excellent scientists and wonderful people. Dr. Clifton Dalgard kindly provided assistance with real-time PCR support and RNA sequencing data analysis. I would like to thank all of my committee members for their guidance and support. None of this would have been possible without the love and support of my family; I want to acknowledge my wife and children for their patience throughout this endeavor. Finally, I would like to acknowledge the United States Army Medical Service Corps and the NIH/NIAID grant RO1-AI42053 for funding this project. 


\section{DEDICATION}

This dissertation is dedicated to the memory of my grandmother. 
The author hereby certifies that the use of any copyrighted material in the thesis manuscript entitled:

"Insights into the Enhanced in vivo Fitness of Neisseria gonorrhoeae Driven by a Fluoroquinolone Resistance-Conferring Mutant DNA Gyrase"

is appropriately acknowledged and, beyond brief excerpts, is with the permission of the copyright owner.

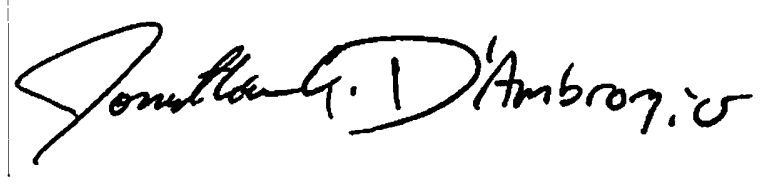

Jonathan A. D'Ambrozio

Molecular and Cell Biology Ph.D. Program

Uniformed Services University

02/09/2015 


\begin{abstract}
Title of Dissertation:

"Insights into the Enhanced in vivo Fitness of Neisseria gonorrhoeae Driven by a Fluoroquinolone Resistance-Conferring Mutant DNA Gyrase”
\end{abstract}

Jonathan Andrew D’Ambrozio, Doctor of Philosophy, 2015

Thesis directed by:

Ann E. Jerse, Ph.D.

Professor, Department of Microbiology and Immunology

With no vaccine available, control of gonorrhea is seriously threatened by the evolution of heightened antibiotic resistance. The fluoroquinolone class of antibiotics served as a first-line treatment option for gonorrhea for only a limited period of time due to the proliferation of resistance mutations. Resistance to fluoroquinolones in Neisseria gonorrhoeae is achieved in a two-step process in which mutations in the quinolone resistance-determining regions of the genes encoding GyrA and ParC confer intermediate and full resistance, respectively. A clinically relevant $g_{y} \mathrm{~A}_{91 / 95}$ mutation was previously demonstrated by our laboratory to confer increased in vivo fitness to $N$. gonorrhoeae strain FA19, leading us to hypothesize that the resistance-conferring $\operatorname{gyr}_{91 / 95}$ mutations are sufficient to alter the in vivo fitness of other $N$. gonorrhoeae strains. We found that the presence of this allele in two additional strains of $N$. gonorrhoeae (FA1090 and 
MS11) not only enhanced in vivo fitness, but did so at a level that was 10-fold higher than that reported in strain FA19. We further speculated that the enhanced in vivo fitness phenotype arose through the altered enzymatic behavior of mutant DNA gyrase, leading to a transcriptional profile better suited for bacterial survival or growth within the host. To test this hypothesis, transcriptome signatures were captured and compared between mutant and parent bacteria using total RNA sequencing as a means of identifying potential mechanisms of gyrA $_{91 / 95}$-driven enhanced in vivo fitness. In vitro assays were also conducted to compare wild-type and gyrA mutant bacterial phenotypes that might explain the enhanced fitness phenotype. We showed that genome-wide expression patterns differed across three sets of mutant and parent strains. Additionally, the mutant that showed the most robust enhancement in fitness relative to its parent strain was also more resistant to human and bacterial antimicrobial peptides, including the cathelicidins and $\mathrm{H}_{2} \mathrm{O}_{2}$ in vitro, and the fitness advantage of this mutant was not observed in cathelicidin-deficient mice. Collectively, these results suggest that a fluoroquinolone resistance-conferring mutations in GyrA condition the N. gonorrhoeae genome for increased survival or growth in vivo, and in the case of one strain background, these mutations increase resistance to host assault, allowing enhanced survivability over susceptible wild type bacteria. 


\section{TABLE OF CONTENTS}

Insights into the Enhanced in vivo Fitness of Neisseria gonorrhoeae Driven by a

Fluoroquinolone Resistance-Conferring Mutant DNA Gyrase ...................................... i

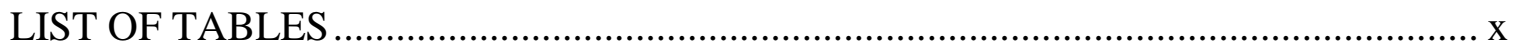

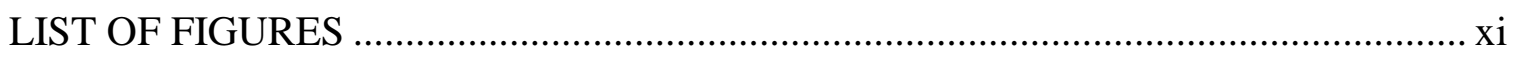

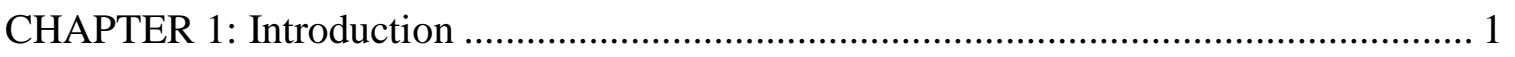

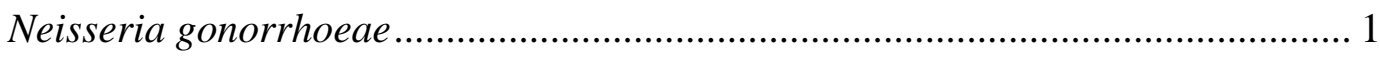

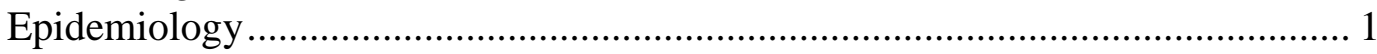

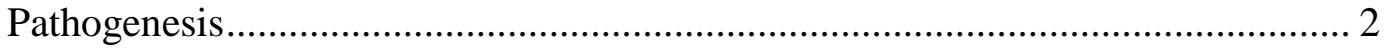

Virulence factors and evasion of host defenses ............................................... 3

Experimental models of infection ............................................................. 7

Diagnosis and Treatment ........................................................................ 9

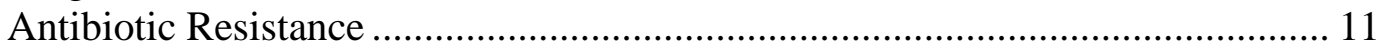

Type II Topoisomerase and the Quinolone Resistance-Determining Region ...... 14

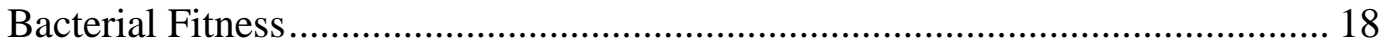

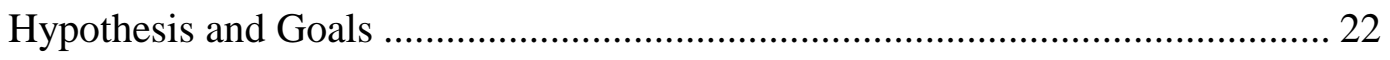

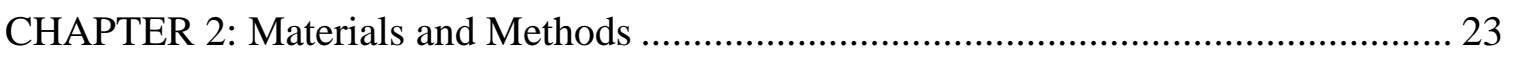

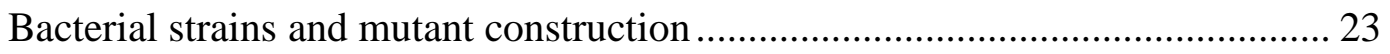

In vitro growth measurements ............................................................ 30

Minimum Inhibitory Concentration (MIC) measurements................................ 31

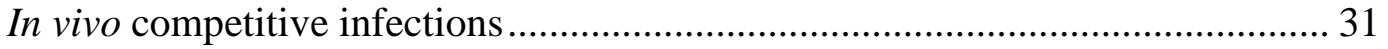

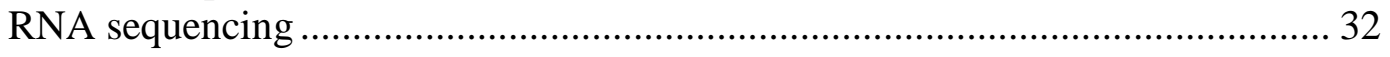

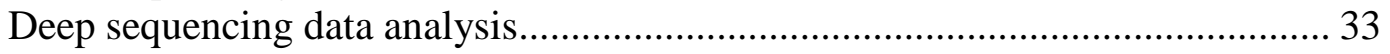

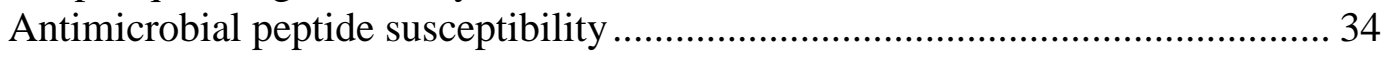

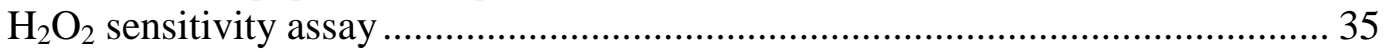

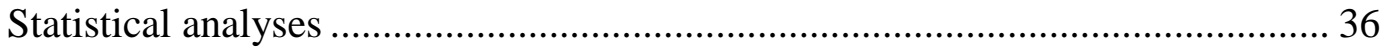

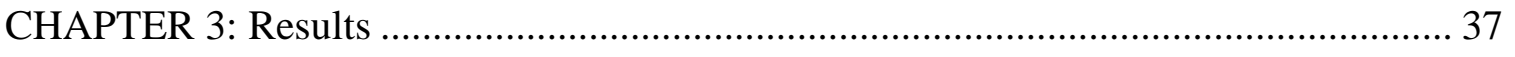

A gyrA allele containing Ser91Phe and Asp95Asn substitutions confers intermediate level ciprofloxacin resitance to two other gonococcal strains ......... 37

Growth measurements and in vivo competitive infections ............................... 39

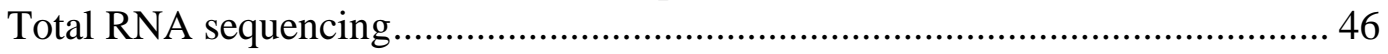

In vitro growth in the absence of co-carboxylase .......................................... 56

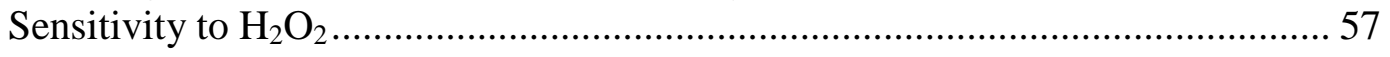

Sensitivity to cationic antimicrobial peptides .............................................. 57

In vivo competitive infection in wild-type and $\mathrm{CRAMP}^{-/} \mathrm{BALB} / \mathrm{c}$ mice............. 58

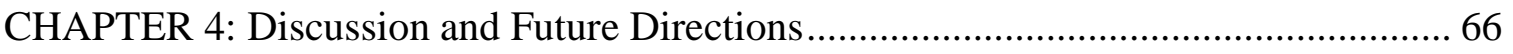


Appendix: The Impact of Fluoroquinolone Resistance-conferring gyrA and parC Mutations on Fitness and the Role of Compensatory Mutations ....................................... 75

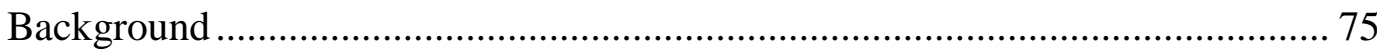

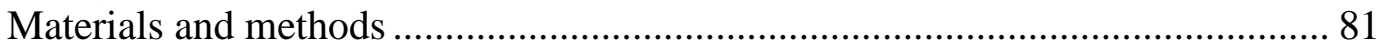

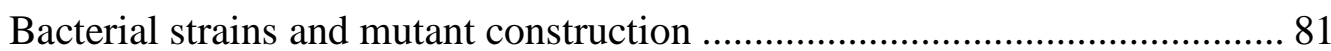

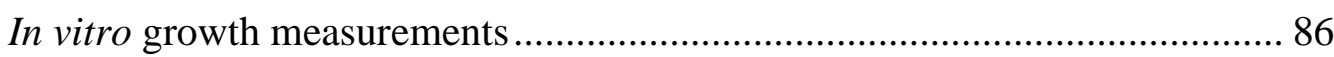

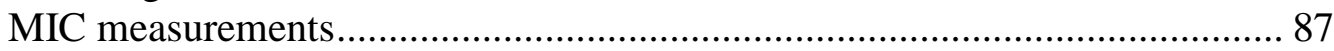

In vivo competitive infections .................................................................. 88

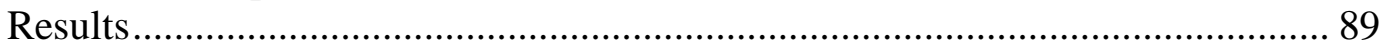

Discussion and Future Directions ..................................................................... 95

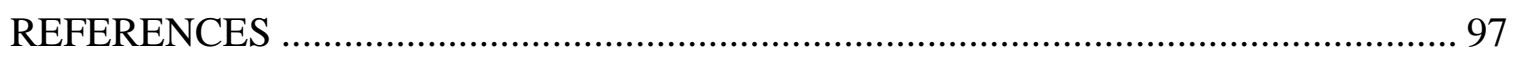




\section{LIST OF TABLES}

Table 1. List of parent strains and mutants used throughout these studies.

Table 2. Oligonucleotide primers used for strain construction and sequencing (5' to 3')

Table 3. Characteristics of the RNA sequencing analysis for each strain...................... 38

Table 4. Up-regulated genes associated with the biosynthesis of thiamine. .................. 49

Table 5. Up-regulated putative genes involved in gene regulation............................. 50

Table 6. Up-regulated genes associated with general metabolism and DNA damage and

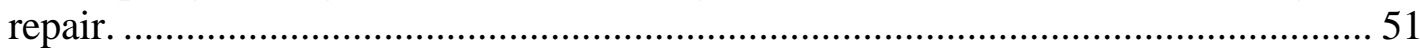

Table 7. Up-regulated genes associated with iron utilization or transport..................... 52

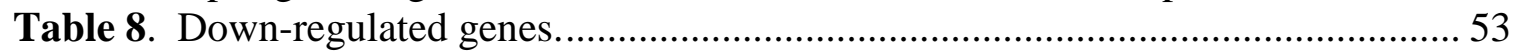




\section{LIST OF FIGURES}

Figure 1. Acquisition of intermediate level resistance ( $\left.\mathrm{Cip}^{\mathrm{I}}\right)$ and high level resistance $\left(\mathrm{Cip}^{\mathrm{R}}\right)$ to fluoroquinolones in $N$. gonorrhoeae occurs in a step-wise manner.......... 16

Figure 2. Fitness studies with $\operatorname{Cip}^{\mathrm{R}} N$. gonorrhoeae using the mouse model of experimental infection. Results showed that the $\mathrm{Cip}^{\mathrm{R}}$ mutant carrying an additional antibiotic resistance mutation in the mtr locus exhibited a fitness disadvantage relative to $\mathrm{Cip}^{\mathrm{I}}$ or $\mathrm{Cip}^{\mathrm{S}}$ bacteria. Restored or enhanced fitness can be achieved

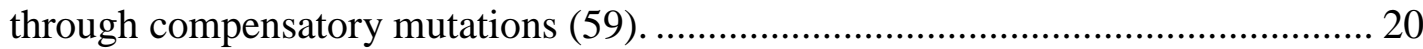

Figure 3. gyrA nucleotide sequence from mutant JD1............................................ 26

Figure 4. gyrA nucleotide sequence from mutant JD4............................................. 28

Figure 5. Growth kinetics of each gyr $A_{91 / 95}$ mutant versus its isogenic parent strain when

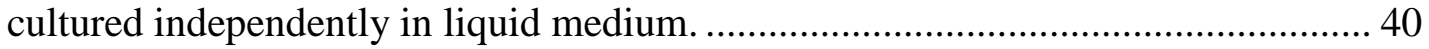

Figure 6. In vitro co-cultures of gyr $A_{91 / 95}$ mutant and wild-type parent strains............. 42

Figure 7. Three different gyr $A_{91 / 95} \operatorname{Cip}^{\mathrm{I}}$ mutants out-compete their $\mathrm{Cip}^{\mathrm{S}}$ wild-type parents in an in vivo infection model. ............................................................... 44

Figure 8. Heat signatures for comparative transcriptome expression profiles................ 54

Figure 9 The gyr $A_{91 / 95}$ mutant in the MS11 background displayed decreased sensitivity to hydrogen peroxide.

Figure 10. The gyrA $A_{91 / 95}$ mutant in the MS11 background displayed decreased sensitivity to CRAMP.

Figure 11. Competitive infections in CRAMP-sufficient (wild-type) and CRAMPdeficient BALB/c mice.

Figure 12. Highlighted QRDR GyrA and ParC amino acid sequences of $\operatorname{Cip}^{\mathrm{S}}$ and $\mathrm{Cip}^{\mathrm{R}}$ Neisseria gonorrhoeae.

Figure 13. Open reading frame sequence of the $\operatorname{par} C$ sequence from JD1.2 ................ 83

Figure 14. Open reading frame sequence of the parC sequence from JD4.2 ................ 85

Figure 15. Growth curves for MS11, FA1090, and the two $\operatorname{Cip}^{\mathrm{R}}$ mutants .................... 91

Figure 16. In vivo assessment of fitness between parental bacteria and the $g y r A_{91 / 95}$ and $\operatorname{parC}_{86} \operatorname{Cip}^{\mathrm{R}}$ mutants 


\section{CHAPTER 1: Introduction}

\section{Neisseria gonorrhoeae}

The majority of the 19 species within the genus Neisseria are human commensal bacteria. Neisseria gonorrhoeae, along with Neisseria meningitidis are the two species capable of causing disease in humans. First characterized by Albert Neisser in 1879, $N$. gonorrhoeae is a Gram-negative, non-motile, non-encapsulated diplococcus that has been exclusively adapted to the human host over thousands of years (81). Although other sites of infection exist, $N$. gonorrhoeae infections most commonly occur in the urogenital tract. With no external environmental or animal reservoir, N. gonorrhoeae has adapted to hostile host responses in humans through the evolution of mechanisms that counter these defenses. Antigenic modulation of the bacterial cell surface is a hallmark feature of $N$. gonorrhoeae, and this is one mechanism that helps the pathogen evade host defense

mechanisms. Additional mechanisms include the capacity to evade innate effectors through surface structure modulation and the ability to utilize human iron-binding glycoproteins for efficient iron acquisition at iron-limited mucosal surfaces.

\section{Epidemiology}

$N$. gonorrhoeae infections are a global concern. The most recent data compilation from the World Health Organization on sexually transmitted infections reveals that over 106 million new cases of $N$. gonorrhoeae arose in 2008, which was estimated to be a 
$21 \%$ increase from three years prior (2). More recent estimates from the Center for Disease Control state that $N$. gonorrhoeae infections are the second most commonly reported infections in the United States. In 2012 alone, a total of 334,826 cases were reported in the U.S., representing a $4.1 \%$ increase from the year prior (1). The southern region of the United States carried the greatest burden of infection, followed by the Midwest, Northeast, and the Western parts of the country. Rates of infection are highest among adolescents and young adults, and tend to be slightly higher in women than men since 2001; however, the rate of increase in men was greater than 100 -fold compared to that of women from 2011 to 2012 (1).

\section{Pathogenesis}

The urogenital epithelium are the primary site of $N$. gonorrhoeae infection. The uterine cervix and the urethra are the sites of initial infection in women and men, respectively. However, other common sites of infection include the pharynx and rectal mucosa, and in newborns, the conjunctiva. It is estimated that up to 4,000 newborn babies become blind annually worldwide due to eye infections resulting from untreated maternal gonococcal and chlamydial infections (2).

Gonococcal urogenital tract infections manifest differently in men and women. In men, a minor percentage of individuals may develop asymptomatic disease progression, but acute urethritis is the most common outcome. Influx of polymorphonuclear neutrophils (PMNs) and urethral epithelial cell shedding contribute to the mucopurulent discharge that is characteristic of symptomatic gonococcal disease and is the defining 
feature of infection in men (26; 81). Symptomatic disease in men can lead to complications such as epididymitis, prostatitis, and infertility. In contrast, both symptomatic and asymptomatic cervical infections can lead to complications, and the overwhelming burden of asymptomatic infection is carried by women (26; 28; 32).

Gonococcal infection of women of child-bearing age most commonly presents as cervicitis. Unlike the singular hallmark feature gonococcal urethritis in men, there is significant variation in the clinical presentation of cervicitis (14; 26; 28). Features of infection may include vaginal bleeding, dysuria, vaginal discharge, erythema and edema, and menorrhagia. As high as $80 \%$ of women with cervical infections are asymptomatic (26). The common asymptomatic nature of gonococcal infection in women increases the likelihood of undiagnosed disease, which can facilitate progression to ascending infection in the upper genital tract. A major consequence of ascending gonococcal infection includes pelvic inflammatory disease (PID), which is often followed by long-term pelvic pain, ectopic pregnancy, and infertility. It is estimated that up to $45 \%$ of females with gonorrhea will develop PID. Furthermore, up to $90 \%$ of women with disseminated gonococcal infection present no indication of associated genital tract infection (26; 28; $32)$.

\section{Virulence factors and evasion of host defenses}

The genital mucosa contains a plethora of innate immune effectors that $N$. gonorrhoeae must successfully evade in order to establish infection in the human host. 
Antimicrobial peptides, reactive oxygen species, and complement present challenges for effective gonococcal colonization.

Several virulence factors have been identified and studied in $N$. gonorrhoeae as mechanisms by which the gonococcus evades immune defenses. Lipooligosaccharide (LOS), pilus, and opacity-associated (Opa) outer membrane proteins are key contributors to the pathogen's successful adaptation to the human host (26; 30). Host immune evasion is enhanced through alterations in the expression of these surface-associated virulence factors, and the alterations are achieved through phase or antigenic variation (22; 105).

The biosynthesis of the gonococcal LOS is achieved through the activity of multiple LOS glycosyl transferase (lgt) genes resulting in a branched oligosaccharide molecule, which is anchored to the membrane by lipid A (74; 105). Three carbohydrate chains $(\alpha, \beta$, and $\gamma)$ display considerable variation, even within the same strain of $N$. gonorrhoeae, due to the high frequency of lgt gene phase variation (22; 88). Gonococcal LOS is involved in a range of activities that contribute to disease, including bacterial attachment to epithelial cells, the induction of cytokines and chemokines leading to PMN recruitment, and human fallopian tube damage (26). Modifications to LOS structure also promote gonococcal evasion of host defenses. For example, sialylation of gonococcal LOS is achieved through addition of host-derived neuraminic acid to the terminal galactose of the lacto- $N$-tetraose moiety of the LOS $\alpha$-chain. Gonococcal LOS sialylation confers protection against LOS-specific antibodies and decreases susceptibility to human PMN killing (102).

Additionally, decoration of the 4' position of lipid A with phosphoethanolamine (PEA) confers increased resistance to complement and antimicrobial peptides. Like 
gonococcal LOS sialylation, PEA decoration of lipid A confers protection against a range of host defenses. PEA can also be added to the heptose sugar within the $\beta$-chain of the primary oligosaccharide, but this modification does not affect serum resistance or resistance to antimicrobial peptides (50; 102). In contrast, PEA-decorated lipid A provides another mechanism of resistance to antimicrobial peptide killing (50; 63).

The gonococcal type IV pilus mediates attachment to non-ciliated secretory epithelial cells, provides motility for early bacterial distribution throughout the surface of the epithelium, and facilitates bacterial aggregation. Several genes encode various subunits of the type IV pili, and the PilE protein (encoded by pilE) is the major pilus subunit. Antigenic variation of PilE occurs as a result of recombination events between pilE and one of several copies of the silent pilS loci (54).

Another N. gonorrhoeae virulence factor that is involved in facilitating the interaction between the pathogen and host is the Opa protein family. These gonococcal surface proteins aid infection by elevating resistance to complement (19). Unlike other outer membrane surface components, the Opa protein family is thought to be responsible for gonococcal association with a broad range of host cell types. Like the gonococcal LOS, expression of Opa proteins is subject to phase variation (54). Up to 12 opa genes may be present within the genome of a particular strain of $N$. gonorrhoeae and as many as 4-5 proteins may be expressed simultaneously $(38 ; 54)$. Sequence variability among Opa proteins is greatest within two central loops. These regions, referred to as hypervariable domain 1 (HV-1) and hypervariable domain 2 (HV-2), confer the tropism for certain cell types exhibited by different Opa proteins (38). 
Efflux pump systems are present in most cells, and provide bacteria with a means of toxic elimination. Expulsion of a range of hydrophobic compounds including synthetic antibiotics and host-derived antimicrobial peptides eliminates or limits the lethality of these agents. Efflux pump systems fall into several families: the resistancenodulation-cell division (RND) family, the ATP-binding cassette (ABC) family, the small multi-drug resistance family, the major facilitator (MF) family, and the multidrug and toxic compound extrusion (MATE) family (68). N. gonorrhoeae has four known efflux pumps including the FarA-FarB system (MF), NorM (MATE), the MacA/MacB system, and the multiple transferable resistance (MtrCDE) system, which is under the influence of an activator (MtrA) as well as a repressor (MtrR) (97). The FarA-FarB system recognizes and eliminates antimicrobial long-chain fatty acids. NorM provides the gonococcus with decreased susceptibility to several antibiotics that have quaternary ammonia structures (68). The MtrCDE system recognizes and exports certain antibiotics, hydrophobic agents, bile salts, steroidal hormones, and nonionic detergents (68). Susceptibility to host phagocytic or epithelial-induced cationic antimicrobial peptides/proteins (CAMP) is decreased in N. gonorrhoeae through the activity of the MtrCDE efflux pump.

The migration of PMNs to the site of infection, engulfment of bacteria, and elimination of microbes during an inflammatory response involves mechanisms that employ oxygen-dependent killing. Activation of PMNs leads to an oxidative burst catalyzed by the activity of NADPH oxidase within the phagosome membrane; however, N. gonorrhoeae is able to survive and replicate within host PMNs (82). 
N. gonorrhoeae has several antioxidant systems. For example, production of gonococcal catalase has been shown to be critical for $\mathrm{H}_{2} \mathrm{O}_{2}$ tolerance in vitro and the MntABC-type transporter uses the manganous ion $\left(\mathrm{Mn}^{2+}\right)$ as a means of quenching reactive oxygen species (ROS) $(71 ; 72)$. The gonococcal cytochrome c peroxidase and methionine sulfoxide reductase enzymes both provide protection against $\mathrm{H}_{2} \mathrm{O}_{2}$ killing (71; 72). However, while clearly important in the protection against oxidative stress in vitro, none of the mechanisms described above lead to evasion of PMN-produced ROS (103) (39; 79).

\section{Experimental models of infection}

Since natural infection with $N$. gonorrhoeae is exclusive to the human host, investigating the dynamics of pathogenesis from both the host and bacterial perspective presents challenges. Studies with male volunteers have provided a wealth of insight into the early stages of urethral infection. These studies have also facilitated the understanding of gonococcal virulence factors and the susceptibility to reinfection as well as provided information regarding vaccine candidates. However, these studies are limited to the urethral epithelium in males, and events that define the course of infection in females cannot be implied. Primary human cell cultures have been utilized to investigate gonococcal involvement in the upper and lower genital tracts, yet, these cell culture systems are restricted to cell type and fail to encapsulate the range of pathogenesis that occurs throughout the female genital tract. 
Early animal models of gonococcal infection included the extragenital inoculation of rabbits and mice in the 1930s and 1940s; however, the use of animals to study gonococcal disease ceased throughout the middle part of the $20^{\text {th }}$ century $(8 ; 9 ; 21)$. Investigations of gonococcal arthritis and disseminated infection were enhanced through the use of rabbits and small rodents throughout the 1970s and 1980s (11), and while these models provided valuable insight into the nature of extragenital gonococcal infections, there were no animal models of gonococcal genital tract infection until chimpanzees were utilized in pathogenesis and sexual transmission studies (10). Unfortunately, the use of non-human primates presents considerable fiscal constraints, which has led some investigators to pursue murine models of gonococcal infection.

Studies in the 1980s provided evidence that female mice could be infected with $N$. gonorrhoeae during proestrous, which is a $12-24$ hour period of increasing estrogen during the murine estrous cycle (41). This window of susceptibility was extended through the use of $17 \beta$-estradiol injections to establish prolonged gonococcal infection in gnotobiotic BALB/c female mice (86). In 1999, our laboratory confirmed the findings of this effort and refined the technique with the use of antibiotics to eliminate the inhibitory influence of vaginal flora. In our current protocol, which uses a series of three subcutaneous injections of water-soluble estradiol, colonization is maintained in female BALB/c mice for an average of 10 days (41). Following vaginal inoculation with approximately $10^{6}$ colony forming units (CFUs) of $N$. gonorrhoeae, an average of $10^{3}$ to $10^{5} \mathrm{CFUs}$ are recovered from a single vaginal swab. It is not known exactly why estradiol is required for long-term colonization in mice; however, suppression of PMN 
influx, alteration of immune receptors, and a diminished cytokine production may be responsible (41).

\section{Diagnosis and Treatment}

Nucleic Acid Amplification Tests (NAATs) are recommended for the detection of N. gonorrhoeae genital tract infections in men and women. Vaginal swabs from women and first catch urine from men are the specimens of choice for NAAT-based detection of N. gonorrhoeae (23). Although not cleared by the Food and Drug Administration for extragenital sites, NAAT detection is utilized in the clinical laboratory with established performance specifications under the Clinical Laboratory Improvement Amendments (CLIA) regulatory guidance. Traditional culture methods are employed when antimicrobial susceptibility testing is needed in the case of treatment failure. Culturebased detection is also needed in cases of possible sexual assault in children (23).

The management of gonorrhea infection rests exclusively on antibiotic treatment. Prior to the success of sulfa drug treatment for gonococcal infections in the late 1930's, urethritis in men and cervicitis in women were often treated with cumbersome injections of potassium permanganate and organic silver salt into the urethra and vagina (43). Antibiotic treatment first began with sulfonamide circa 1937, and this approach was followed by the penicillin era in the early 1940s, which led to a significant decline in gonococcal infections (43). However, in the forty years that have followed, $N$. gonorrhoeae has established itself as an organism with the capacity to develop resistance to all antibiotics that have thus far been recommended for treatment. Now, with 
resistance emerging to the third-generation cephalosporins, a dual regimen of injectable ceftriaxone in combination with one of two oral antibiotics, either azithromycin or doxycycline, as the lone treatment option for gonorrhea (1).

\section{Gonococcal Vaccine Development}

The global prevalence of gonococcal infections, continued proliferation of multiclass antibiotic resistance, and the devastating sequelae associated with asymptomatic infections in women demand a need for gonococcal vaccine development. However, several factors have contributed to a relatively conservative approach to $N$. gonorrhoeae vaccine research. While a significant health concern, gonorrhea may not be viewed by many as a threat of the level of other diseases. Gonococcal infections lack the high profile lethality of meningococcal disease, which may limit the wide-spread public backing needed to help elevate research toward a gonococcal vaccine. Additional factors that have limited research progression in this field include the highly variable nature of $N$. gonorrhoeae surface components as discussed earlier, a lack of evidence in support of naturally-acquired immunity, and undefined correlates of protection (18; 24; 40).

To date, the few gonococcal vaccines that have advanced to clinical trials have been unsuccessful. The utility of a killed whole cell vaccine was investigated on a population in Northern Canada (33; 34). However, no protection to subsequent challenge was observed, and further exploration of whole cell gonococcal vaccines ceased. As mentioned previously, the gonococcal type IV pili mediate attachment, afford motility, and mediate bacterial aggregation during infection. Considerable attempts aimed at 
developing a purified pilin vaccine showed early promise. Studies in human volunteers generated an immunogenic response, with blocking antibodies observed in both serum and genital secretions (53; 76; 89). Using a $N$. gonorrhoeae pilin vaccine, a large-scale field trial was carried out in the Republic of South Korea on 3123 male and 127 female

U.S. military active duty volunteers (20). The study led to no evidence of protection, and the failure of this single-antigen vaccine trial has been attributed to the highly variable nature of the gonococcal pilin. No additional vaccine candidates have advanced to clinical trials since the failures of the whole cell killed and pilin vaccines.

The absence of any promising vaccine candidates currently in product pipeline and the accelerating risk of untreatable gonorrhea through the spread of ceftriaxone resistance provides a formidable challenge to public health.

\section{Antibiotic Resistance}

Just as the sulfonamides were fading as a successful treatment option, the discovery of penicillin and its effectiveness as a therapy for gonococcal infections ushered in a new treatment era (95). The accidental discovery of penicillin in the late 1920’s by Alexander Fleming was slow to catch on as a treatment for gonococcal infections, but by 1943 the use of penicillin was an efficient and effective means for managing gonorrhea infection. However, not long after surpassing the sulfonamides as the means of primary therapy for gonorrhea, penicillin resistant determinants started to emerge. The development of later classes of antibiotics, such as the macrolides, aminoglycosides, and the tetracyclines, provided a means of circumventing intolerance to 
penicillin in some infected individuals; however, by the time penicillin resistance became wide-spread and was no longer recommended for the treatment of gonorrhea, $N$. gonorrhoeae had also acquired either plasmid or chromosomally-mediated resistance mutations to several of these antibiotics, including streptomycin erythromycin, tetracycline, and spectinomycin (52; 78; 87; 96).

Penicillins disrupt the dynamic process of bacterial cell wall metabolism; however, $N$. gonorrhoeae has acquired a diverse set of chromosomally mediated and plasmid-encoded determinants that confer resistance to penicillins. Mutations in the penA and ponA genes, which encode the primary and secondary targets of penicillin, penicillin binding proteins 2 and 1 (PBP2 and PBP1) respectively, lead to decreased penicillin-directed acylation (95; 96). Mutations that lead to over-expression of the MtrCDE efflux pump lead to decreased drug sequestration. Reduction in penicillin influx is also associated with porB1b single nucleotide polymorphisms (SNPs) and pilQ mutations that disrupt the formation of the type IV pilus. The TEM-1 and TEM-135 penicillinase-encoding plasmids directly inactivate penicillin through an attack on the $\beta$ lactam ring. Finally, "Factor X” is an unknown resistant determinant associated with up to 6-fold increases in penicillin Minimum Inhibitory Concentrations (MICs) (61; 95; 96).

As mentioned, the tetracycline class of antibiotics provided an effective means of treating gonorrhea for individuals with penicillin sensitivities. Tetracycline targets the 30 S ribosomal subunit; however, chromosomal mutations in the rps $J$ gene of $N$. gonorrhoeae lead to diminished target affinity. Acquisition of tetM-encoding plasmids further increases MICs to tetracycline by producing an elongation factor-like protein that prevents drug association (95; 96). Like penicillin resistance, increased MtrCDE efflux 
pump expression and pilQ and penB resistance determinants that decrease drug influx also raise tetracycline MICs.

Like tetracycline, spectinomycin blocks bacterial protein translation through an inhibitory association with the 30 S ribosomal subunit. Specifically, inhibition of bacterial elongation factor $\mathrm{G}$ (EF-G) is achieved by direct association of spectinomycin with the 16S rRNA (94). Mutations in the gonococcal rpsE gene that alter the amino acid sequence of the S5 ribosomal protein decrease drug binding (52; 87; 96) .

Resistance to macrolides, which inhibit bacterial protein synthesis through $50 \mathrm{~S}$ subunit targeting, is achieved in the gonococcus through decreased drug association with the ribosomal complex. This decreased drug-target association occurs via mutations in the 23S rRNA that alter the amino acid composition, or through the acquisition of macrolide-lincosamide-streptogramin B resistance genes (erm), which encode rRNA methylases (57). The methylation of 23S rRNA masks the macrolide binding site. Mutations that lead to de-repression of the MtrCDE efflux pump also raise macrolide $\operatorname{MICs}(25 ; 96)$.

The development of the broad-spectrum fluoroquinolone class of antibiotics emerged as an attractive therapeutic alternative in the wake of resistant strains of bacteria in the early 1980s (27) (95). This bactericidal class of antibiotic targets Type II topoisomerases in the cell and inhibits the enzyme in a mechanism thought to involve a DNA-bound complex $(5 ; 64 ; 104)$. The prevailing model of inhibition involves binding of the fluoroquinolone molecules to the DNA enzyme complex and accumulation of a cleaved DNA-enzyme lethal intermediate (104). By the mid-1980s, ciprofloxacin became a popular antibiotic therapy for $N$. gonorrhoeae infections after the CDC reported 
rising numbers of plasmid-mediated resistance to tetracycline and penicillinase-producing gonococcal isolates (55). Unfortunately, although highly effective in the treatment of gonorrhea, increased MICs to ciprofloxacin were being reported in Southeast Asia as early as 1988. The first clinically resistant isolates of $N$. gonorrhoeae were reported in Australia, and by the late 1990s, the percentage of clinically resistant isolates in Hawaii rose to $9.5 \%$ (22 of 231) $(91 ; 95)$. By 2000 , over $80 \%$ of the gonococcal isolates from Western Pacific Region were clinically resistant to ciprofloxacin. Clinically resistant isolates continued to move west, and by 2007, 14.5\% (891 of 6009) of gonococcal isolates in the United States were clinically resistant (56; 91), and in 2007, the CDC discontinued the recommendation of fluoroquinolones as a primary treatment option for gonorrhea. In summary, resistance to the fluoroquinolones occurred after a relatively short duration of recommended use, and the loss of fluoroquinolone use exemplifies the rapid emergence of antibiotic resistance in the gonococcus (45).

\section{Type II Topoisomerase and the Quinolone Resistance-Determining Region}

As mentioned above, the targets of fluoroquinolones are the heterotetramer Type II topoisomerase enzymes DNA gyrase and topoisomerase IV. The genes encoding the individual subunits of these heterotetramers are gyrA and $\operatorname{grB}$ (DNA gyrase) and parC and parE (topoisomerase IV) $(90 ; 101)$. The state of genomic topology plays an important role during the key molecular events of transcription and DNA replication, where supercoiling of the DNA is altered to accommodate these biological processes (77). The type II topoisomerases are responsible for managing the genomic topology in 
the bacterial cell, and this is achieved by an ATP-dependent, double-stranded cleavage of the DNA duplex. While both DNA gyrase and topoisomerase IV share the same mechanistic approach for influencing the degree of DNA supercoiling, DNA gyrase functions primarily to increase negative supercoiling, while topoisomerase IV is mainly involved in the separation of sister chromatids during DNA replication (15). As mentioned previously, it took less than 20 years before the fluoroquinolone class of antibiotics was no longer a first-line treatment option for $N$. gonorrhoeae. Emergence of fluoroquinolone resistance in $N$. gonorrhoeae was due to acquisition of chromosomal mutations in the quinolone resistance-determining regions (QRDRs) within the gyrA and parC genes (64; 73; 83; 85; 92; 93; 106). Acquisition of these mutations occurs in a stepwise manner (Fig. 1), with a double point mutation in gyrA conferring an intermediate degree of resistance to ciprofloxacin (Cip $\left.{ }^{\mathrm{I}}\right)(95)$. An additional single point mutation in parC leads to high-level resistance to ciprofloxacin $\left(\mathrm{Cip}^{\mathrm{R}}\right)$. 
Figure 1. Acquisition of intermediate level resistance (CipI) and high level resistance (CipR) to fluoroquinolones in N. gonorrhoeae occurs in a step-wise manner.

This illustration represents the steps leading to increased resistance to fluoroquinolones in N. gonorrhoeae with an emphasis on its target (DNA-bound topoisomerase). The susceptible (wild-type) state is represented at the bottom of the figure in which wild-type DNA gyrase is bound and inhibited by fluoroquinolones. A key feature highlighted at the bottom of the figure include the direct association of fluoroquinolone molecules $(\diamond)$ with the nonfunctional DNA gyrase and cleaved DNA duplex intermediate. The images representing DNA gyrase in $\operatorname{Cip}^{\mathrm{I}}$ and $\mathrm{Cip}^{\mathrm{R}}$ strains highlight the lack of association between antibiotic and enzyme. Intermediate-level resistance is most commonly achieved through the acquisition of a double point mutation in gyrA resulting in one of the more frequent amino acid substitutions highlighted. An additional single point mutation in parC establishes a high degree of resistance, and commonly encountered amino acid substitutions associated with this mutant are displayed (59). 


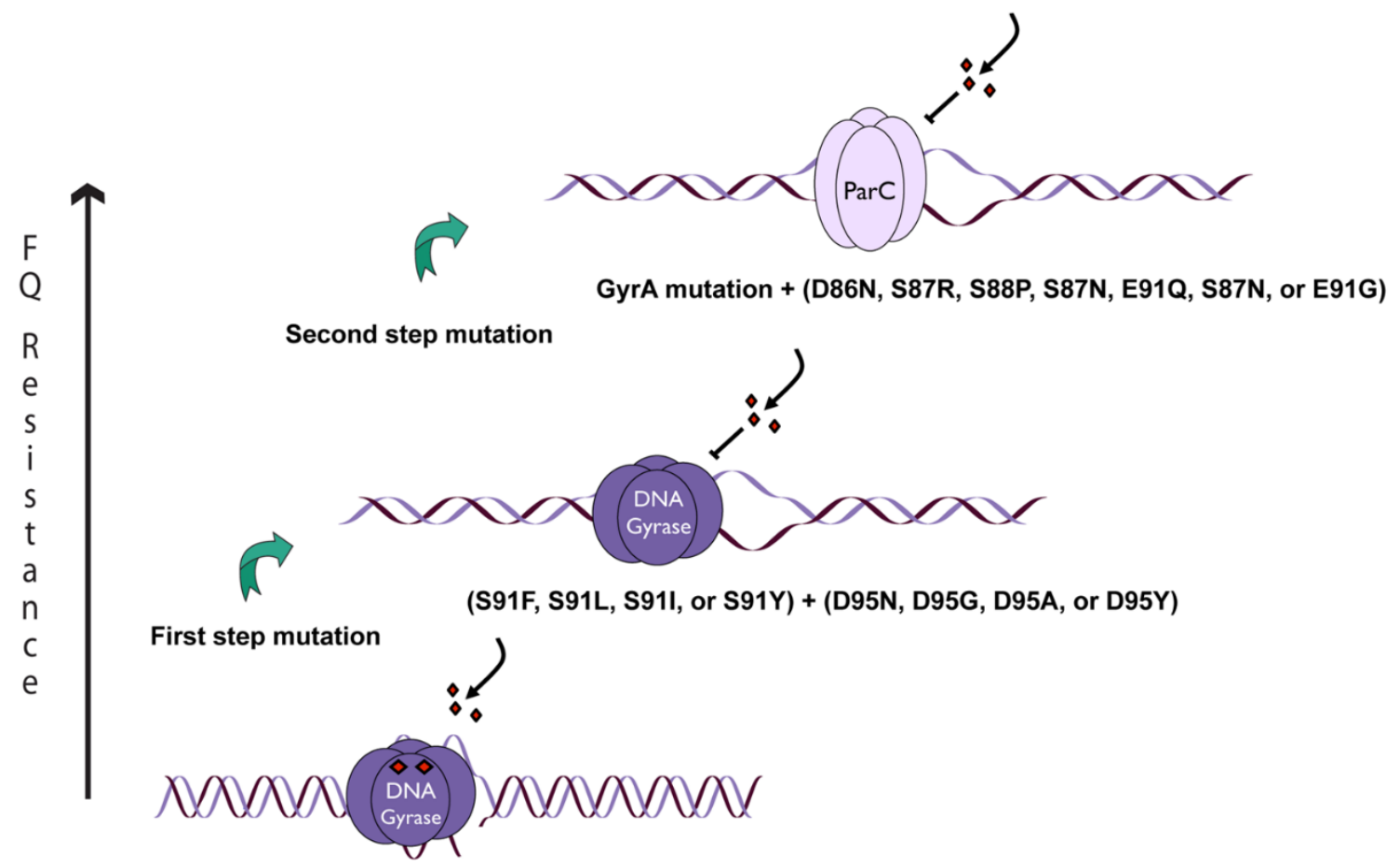




\section{Bacterial Fitness}

Bacterial mutations that decrease the cell's sensitivity to antibiotics have generally been shown to negatively impact bacterial fitness (3; 6; 7; 13). Fanning and colleagues characterized fitness costs in Salmonella enterica resulting from high-level resistance to ciprofloxacin and identified reduced growth rates, motility, and invasiveness as phenotypes in the resistant mutant that might provide a barrier to survival when the antibiotic selective pressure is removed (58). However, studies that link intermediate level fluoroquinolone resistance-conferring mutations with an in vivo fitness advantage have also been reported. In one study, fluoroquinolone-resistant Campylobacter jejuni mutants carrying a single point mutation in the gyrA allele outcompeted fluoroquinolonesensitive strains of the same genetic background in an in vivo chicken model (51). Further studies demonstrated that this point mutation also altered changes in supercoiling (51) (37), although how this observation may cause increased in vivo fitness has not been explained. Studies in E. coli demonstrated that high level fluoroquinolone resistanceconferring mutations in gyrA and parC produced alterations in both growth rate and global supercoiling (12). Furthermore, and similar to the $C$. jejuni study, ciprofloxacin resistance-conferring mutations in the $E$. coli gyrA gene were recently shown to alter the state of genomic supercoiling and the E. coli transcriptome, with several of these changes affecting the expression levels of genes involved in the stress response (100).

In N. gonorrhoeae, we reported that $\operatorname{Cip}^{\mathrm{I}}$ mutants display an enhanced in vivo phenotype in a female mouse model of experimental gonococcal genital tract infection. Using the common laboratory strain of $N$. gonorrhoeae, FA19, we showed that Cip ${ }^{\mathrm{I}}$ 
mutants carrying commonly isolated gyr $_{91 / 95}$ mutations have a fitness advantage when competed against wild type $\operatorname{Cip}^{\mathrm{S}}$ bacteria. However, this in vivo fitness advantage is lost with the acquisition of a common second-site mutation in the $\operatorname{par} C$ gene that is required for high-level resistance to ciprofloxacin (47). Our laboratory also demonstrated the selection of a compensatory mutation during an in vivo competitive infection between $\mathrm{Cip}^{\mathrm{I}}$ and $\mathrm{Cip}^{\mathrm{R}}$ strains that also carry an $m t r$ antibiotic resistance mutation. This result provides evidence that in-host microbial evolution can provide a mechanism for circumventing the fitness costs while maintaining high-level resistance to antibiotics in the absence of selective pressure. The in vivo-selected $\mathrm{Cip}^{\mathrm{R}}$ mutant with an enhanced growth rate relative to parent strain was sequenced and shown to have alterations in gyr $A$ (S91L) and a repaired promoter for a global regulator $(m t r R)$. Interestingly, the gyr $A$ (S91L) mutation has been reported in clinical isolates of $\operatorname{Cip}^{\mathrm{R}} N$. gonorrhoeae (47) (97; 98). A schematic illustrating the competitive infection procedure and the results described above is shown in Fig. 2.

As we continue to move toward an era of untreatable bacterial infections, efforts to establish a fitness value for different resistance-conferring mutations may help predict spread of certain types of resistances. Furthermore, continued characterization of the mechanisms that lead to either a fitness advantage or disadvantage over isogenic sensitive strains may help influence efforts in targeted drug discovery as well as further our understanding of gonococcal adaptation to the host. 
Figure 2. Fitness studies with $\operatorname{Cip}^{\mathrm{R}} N$. gonorrhoeae using the mouse model of experimental infection. Results showed that the CipR mutant carrying an additional antibiotic resistance mutation in the mtr locus exhibited a fitness disadvantage relative to CipI or CipS bacteria. Restored or enhanced fitness can be achieved through compensatory mutations (59). 


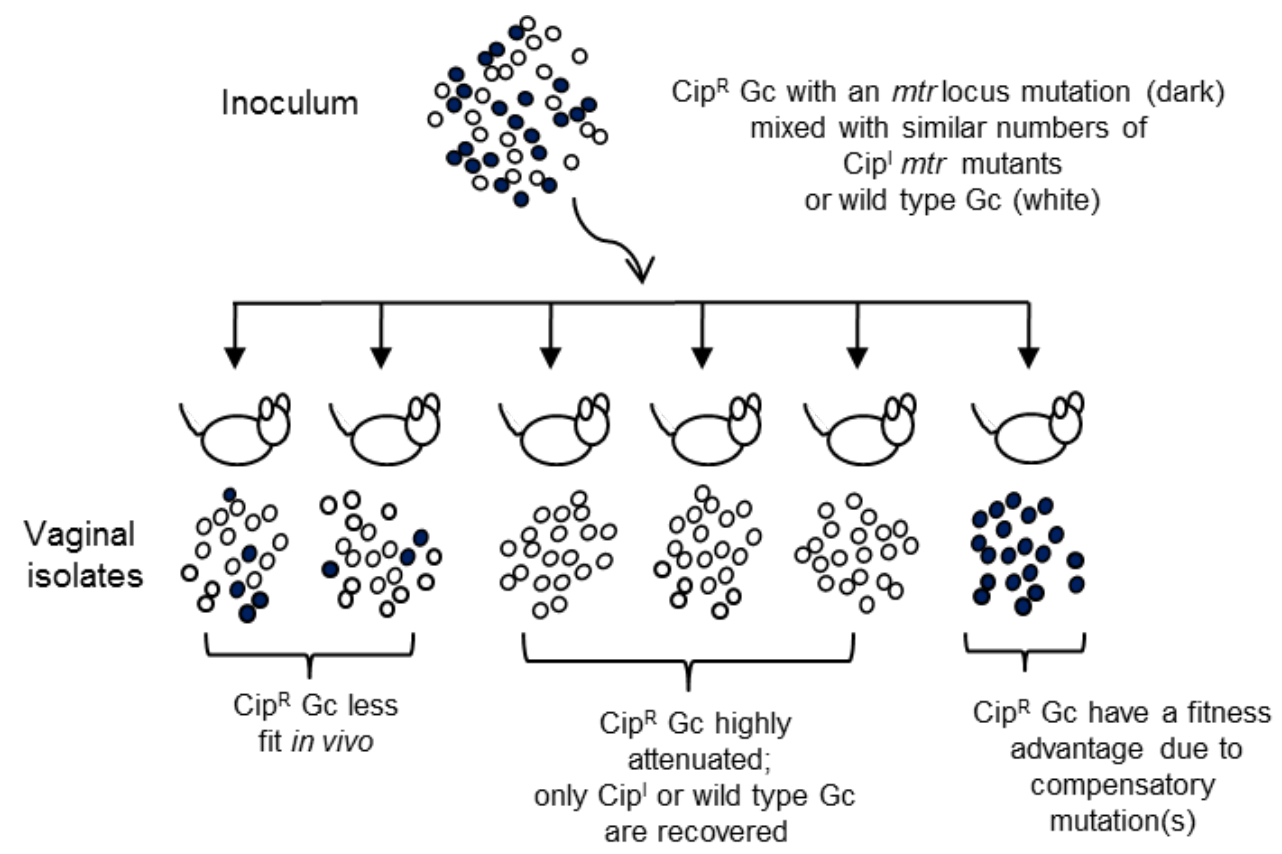




\section{Hypothesis and Goals}

Building on previously reported evidence from our laboratory that $N$.

gonorrhoeae carrying a mutant gyr $A_{91 / 95}$ allele possesses a fitness advantage over wild type sensitive bacteria, the goal of this work was to expand the investigation of the observed enhanced in vivo fitness across multiple strains and to identify the basis of this enhanced fitness by examining transcriptional profiles and conducting in vitro and in vivo assays to measure bacterial resistance to host defenses.

Due to the rapid emergence of fluoroquinolone reistance in $N$. gonorrhoeae, we hypothesized that fluoroquinolone resistance-conferring gyr $A_{91 / 95}$ allele of $N$. gonorrhoeae imparts a strain-independent in vivo fitness advantage. We also hypothesized that this mutation alters the global expression of bacterial genes that results in increased gonococcal survival in vivo. 


\section{CHAPTER 2: Materials and Methods}

\section{Bacterial strains and mutant construction}

Neisseria gonorrhoeae strain FA19Sm ${ }^{\mathrm{R}}$ and its gyrA $_{91 / 95}$ mutant AK1 were described previously (47). N. gonorrhoeae strains FA1090 and MS11 were used to create gyrA $_{91 / 95}$ mutants JD1and JD4, respectively. JD1 and JD4 gyrA91/95 mutants were constructed by allelic exchange with a donor PCR amplicon containing Ser91Phe (TCC to TTC) and Asp95Asn (GAC to AAC) mutations (Table 1). The method employed followed that previously reported for the construction of AK1 (35; 47). Briefly, a 1.3-kb region of gyrA was amplified from AK1 using gyrA1-for and gyrA1-rev primers (Table 2). The resultant amplicon was gel-extracted, and sequenced to confirm the mutated sequence. FA1090 and MS11 bacteria were suspended in PBS and adjusted to an absorbance of 0.05 at 600 nanometers $\left(\mathrm{A}_{600}\right)$. Ten microliters of the bacterial suspension were spotted onto solid non-selective gonococci (GC) agar base media followed by direct overlay of approximately $1 \mu \mathrm{g}$ of the donor DNA. Following incubation at $37^{\circ} \mathrm{C}$ for 24 hours, individual colonies were isolated and streaked onto solid agar containing 0.125 $\mu \mathrm{g} / \mathrm{ml}$ of ciprofloxacin to select for gyr $_{91 / 95}$ mutants. The presence of the desired mutation in colonies that grew on the ciprofloxacin plates was confirmed by sequence analysis (Figures 3 and 4). 
Table 1. List of parent strains and mutants used throughout these studies.

The relevant genotype, fluoroquinolone resistance phenotype, and minimum inhibitory concentrations (MICs) are shown for each parent and mutant strain.

\begin{tabular}{|c|c|c|c|}
\hline$\underline{\text { Strain }}$ & Genotype & Phenotype & MIC (Cip) $(\mu \mathrm{g} / \mathrm{ml})$ \\
\hline FA19Sm ${ }^{R}$ & Spontaneous $\mathrm{Sm}^{\mathrm{R}}$ mutant of wild-type FA19 & $\mathrm{Cip}^{\mathrm{s}}$ & $<0.06$ \\
\hline AK1 & gyrA $_{91 / 95}$ mutant of FA19Sm ${ }^{\mathrm{R}}$ & $\mathrm{Cip}^{\mathrm{I}}$ & 0.25 \\
\hline AK2 & $\operatorname{gyr}_{91 / 95} ;$ par $_{86}$ mutant of $\mathrm{FA} 19 \mathrm{Sm}^{\mathrm{R}}$ & $\mathrm{Cip}^{\mathrm{R}}$ & 6 \\
\hline FA1090 & $m t r A^{-}$ & $\mathrm{Cip}^{\mathrm{S}}$; decreased $m t r C-D-E$ activation & $<0.06$ \\
\hline JD1 & $\operatorname{gyr}_{91 / 95}$ mutant of FA1090 & Cip'; decreased $m t r C-D-E$ activation & 0.38 \\
\hline JD1.2 & gyr $_{91 / 95} ;$ par $\mathrm{C}_{86}$ mutant of FA1090 & $\mathrm{Cip}^{\mathrm{R}}$; decreased $m t r C-D-E$ activation & 6 \\
\hline MS11 & Wild-type strain, $m t r_{120}, m t r R_{\mathrm{A} 39 \mathrm{~T}}$ & $\mathrm{Cip}^{\mathrm{S}}$; naturally de-repressed $m \operatorname{tr} C-D-E$ & $<0.06$ \\
\hline JD4 & $\operatorname{gyr}_{91 / 95}$ mutant of MS11 & Cip; naturally de-repressed $m t r C-D-E$ & 1.5 \\
\hline JD4.2 & $\operatorname{gyr}_{91 / 95} ; \operatorname{parC}_{86}$ mutant of MS11 & $\mathrm{Cip}^{\mathrm{R}}$; naturally de-repressed $m \operatorname{tr} C-D-E$ & 8 \\
\hline
\end{tabular}


Table 2. Oligonucleotide primers used for strain construction and sequencing (5' to 3')

$\begin{array}{ll}\text { gyrA1-for } & \text { GACTTCCTCATGCAGCAAATG } \\ \text { gyrA1-rev } & \text { CAACCATATTGATGCCGAAACTG } \\ \text { gyrA2-for } & \text { ACGAAACATTGAAACCATGAC } \\ \text { parC1-for } & \text { CATAGCGACGGTCTTTGTGTG } \\ \text { parC1-rev } & \text { GTTGATGAAGGTATCGGTATCGATG } \\ \text { parC2-for } & \text { ACGCTTCCCATACCGATTC } \\ \mathbf{5} \text { 'mtrR } & \text { GGTTAATTAACGCCTTAGAAGCATAAAAAGC } \\ \mathbf{3} \text { 'mtrR } & \text { GGGTTTAAACTTATTTCCGGCGCAGGCAG }\end{array}$


Figure 3. gyrA nucleotide sequence from mutant JD1.

The highlighted codons show the TCC $\rightarrow$ TTC point mutation that produces the Ser $\rightarrow$ Phe amino acid transition at position $91(\mathrm{~S} 91 \mathrm{~F})$ and the $\mathrm{GAC} \rightarrow$ AAC point mutation that leads to the Asp $\rightarrow$ Asn amino acid change at position 95 (D95N). 
ATGACCGACGCAACCATCCGCCACGACCACAAATTCGCCCTCGAAACCCTGCCCGTCAGCCTTGAAGACG AAATGCGCAAAAGCTATCTCGACTACGCCATGAGCGTCATTGTCGGGCGCGCGCTGCCGGACGTTCGCGA CGGCCTAAAGCCGGTGCACCGGCGCGTACTGTACGCGATGCACGAGCTGAAAAATAACTGGAATGCCGCC TACAAAAAATCGGCGCGCATCGTCGGCGACGTCATCGGTAAATACCACCCCCACGGCGATTTCGCAGTTT ACAACACCATCGTCCGTATGGCGCAAAATTTCGCTATGCGTTATGTGCTGATAGACGGACAGGGCAACTT CGGATCGGTGGACGGGCTTGCCGCCGCAGCCATGCGCTATACCGAAATCCGCATGGCGAAAATCTCACAT GAAATGCTGGCAGACATTGAGGAAGAAACCGTTAATTTCGGCCCGAACTACGACGGTAGCGAACACGAGC CGCTTGTACTGCCGACCCGTTTCCCCACACTGCTCGTCAACGGCTCGTCCGGTATCGCCGTCGGTATGGC GACCAACATCCCGCCGCACAACCTCACCGACACCATCAACGCCTGTCTGCGTCTTTTGGACGAACCCAAA ACCGAAATCGACGAACTGATCGACATTATCCAAGCCCCCGACTTCCCGACCGGGGCAACCATCTACGGCT TGGGCGGCGTGCGCGAAGGCTATAAAACAGGCCGCGGCCGCGTCGTTATACGCGGTAAGACCCATATCGA ACCCATAGGCAAAAACGGCGAACGCGAAGCCATCGTTATCGACGAAATCCCCTATCAGGTCAACAAAGCC AAGTTGGTCGAGAAAATCGGCGATTTGGTTCGGGAAAAAACGCTGGAAGGCATTTCCGAGCTCCGCGACG AATCCGACAAATCCGGGATGCGCGTCGTTATCGAGCTGAAACGCAACGAAAATGCCGAAGTCGTCTTAAA CCAACTCTACAAACTGACTCCGCTGCAAGACAGTTTCGGCATCAATATGGTTGTTTTGGTCGACGGACAA CCGCGCCTGTTAAACCTGAAACAGATTCTCTCCGAATTCCTGCGCCACCGCCGCGAAGTCGTTACCCGAC GTACGCTTTTCCGGCTGAAGAAGGCACGCCATGAAGGGCATATCGCCGAAGGCAAAGCCGTCGCACTGTC CAATATCGATGAAATCATCAAGCTCATCAAAGAATCGCCCAACGCGGCCGAGGCCAAAGAAAAACTGCTT GCGCGCCCTTGGCGCAGCAGCCTCGTTGAAGAAATGCTGACGCGTTCCGGTCTGGATTTGGAAATGATGC GTCCGGAAGGATTGGCTGCAAACATTGGTCTGAAAAAACAAGGTTATTACCTGAGCGAGATTCAGGCAGA TGCTATTTTACGCATGAGCCTGCGAAACCTGACCGGCCTCGATCAGAAAGAAATTATCGAAAGCTACAAA AACCTGATGGGTAAAATCATCGACTTTGTGGATATCCTCTCCAAACCCGAACGCATTACCCAAATCATCC GTGACGAACTGGAAGAAATCAAAACCAACTATGGCGACGAACGCCGCAGCGAAATCAACCCGTTCGGCGG CGACATTGCCGATGAAGACCTGATTCCGCAACGCGAAATGGTCGTGACCCTGACCCACGGCGGCTATATA AAAACCCAGCCGACCACCGACTATCAGGCTCAGCGTCGCGGCGGGCGCGGCAAACAGGCGGCTGCCACCA AAGACGAAGACTTTATCGAAACCCTGTTTGTTGCCAACACGCATGACTATTTGATGTGTTTTACCAACCT CGGCAAGTGCCACTGGATTAAGGTTTACAAACTGCCCGAAGGCGGACGCAACAGCCGCGGCCGTCCGATT AACAACGTCATCCAGCTGGAAGAAGGCGAAAAAGTCAGCGCGATTCTGGCAGTACGCGAGTTTCCCGAAG ACCAATACGTCTTCTTCGCCACCGCGCAGGGAATGGTGAAAAAAGTCCAACTTTCCGCCTTTAAAAACGT CCGCGCCCAAGGCATTAAAGCCATCGCACTCAAAGAAGGCGACTACCTCGTCGGCGCTGCGCAAACAGGC GGTGCGGACGACATTATGTTGTTCTCCAACTTGGGCAAAGCCATCCGCTTCAACGAATACTGGGAAAAAT CCGGCAACGACGAAGCGGAAGATGCCGACATCGAAACCGAGATTTCAGACGACCTCGAAGACGAAACCGC CGACAACGAAAACACCCTGCCAAGCGGCAAAAACGGCGTGCGTCCGTCCGGTCGCGGCAGCGGCGGTTTG CGCGGTATGCGCCTGCCTGCCGACGGCAAAATCGTCAGCCTGATTACCTTCGCCCCTGAAACCGAAGAAA GCGGTTTGCAAGTTTTAACCGCCACCGCCAACGGATACGGAAAACGCACCCCGATTGCCGATTACAGCCG CAAAAACAAAGGCGGGCAAGGCAGTATTGCCATTAACACCGGCGAGCGCAACGGCGATTTGGTCGCCGCA ACCTTGGTCGGCGAAACCGACGATTTGATGCTGATTACCAGCGGCGGCGTGCTTATCCGTACCAAAGTCG AACAAATCCGCGAAACCGGCCGCGCCGCAGCAGGCGTGAAACTGATTAACTTGGACGAAGGCGAAACCTT GGTATCGCTGGAACGTGTTGCCGAAGACGAATCCGAACTCTCCGGCGCTTCTGTAATTTCCAATGTAACC GAACCGGAAGCCGAGAACTGA 
Figure 4. gyrA nucleotide sequence from mutant JD4.

The highlighted codon shows the TCC $\rightarrow$ TTC point mutation that produces the Ser $\rightarrow$ Phe amino acid transition at position $91(\mathrm{~S} 91 \mathrm{~F})$ and the $\mathrm{GAC} \rightarrow$ AAC point mutation that leads to the Asp $\rightarrow$ Asn amino acid change at position 95 (D95N). 
ATGACCGACGCAACCATCCGCCACGACCACAAATTCGCCCTCGAAACCCTGCCCGTCAGCCTTGAAGACG AAATGCGCAAAAGCTATCTCGACTACGCCATGAGCGTCATTGTCGGGCGCGCGCTGCCGGACGTTCGCGA CGGCCTAAAGCCGGTGCACCGGCGCGTACTGTACGCGATGCACGAGCTGAAAAATAACTGGAATGCCGCC TACAAAAAATCGGCGCGCATCGTCGGCGACGTCATCGGTAAATACCACCCCCACGGCGATTTCGCAGTTT ACAACACCATCGTCCGTATGGCGCAAAATTTCGCTATGCGTTATGTGCTGATAGACGGACAGGGCAACTT CGGATCGGTGGACGGGCTTGCCGCCGCAGCCATGCGCTATACCGAAATCCGCATGGCGAAAATCTCACAT GAAATGCTGGCAGACATTGAGGAAGAAACCGTTAATTTCGGCCCGAACTACGACGGTAGCGAACACGAGC CGCTTGTACTGCCGACCCGTTTCCCCACACTGCTCGTCAACGGCTCGTCCGGTATCGCCGTCGGTATGGC GACCAACATCCCGCCGCACAACCTCACCGACACCATCAACGCCTGTCTGCGTCTTTTGGACGAACCCAAA ACCGAAATCGACGAACTGATCGACATTATCCAAGCCCCCGACTTCCCGACCGGGGCAACCATCTACGGCT TGGGCGGCGTGCGCGAAGGCTATAAAACAGGCCGCGGCCGCGTTGTTATGCGCGGTAAGACCCATATCGA ACCCATAGGCAAAAACGGCGAACGCGAAGCCATCGTTATCGACGAAATCCCCTATCAGGTCAACAAAGCC AAGTTGGTCGAGAAAATCGGCGATTTGGTTCGGGAAAAAACACTGGAAGGCATTTCCGAGCTCCGCGACG AATCCGACAAATCCGGTATGCGCGTCGTTATCGAGCTGAAACGCAACGAAAATGCCGAAGTCGTCTTAAA CCAACTCTACAAACTGACTCCGCTGCAAGACAGTTTCGGCATCAATATGGTGGTTTTGGTCGACGGACAA CCGCGCCTGTTAAACCTGAAACAGATTCTCTCCGAATTCCTGCGCCACCGCCGCGAAGTCGTTACCCGAC GTACGCTTTTCCGGCTGAAGAAGGCACGCCATGAAGGGCATATCGCCGAAGGCAAAGCCGTCGCACTGTC CAATATCGATGAAATCATCAAGCTCATCAAAGAATCGCCCAACGCGGCCGAGGCCAAAGAAAAACTGCTT GCGCGCCCTTGGCGCAGCAGCCTCGTTGAAGAAATGCTGACGCGTTCCGGTCTGGATTTGGAAATGATGC GTCCGGAAGGATTGGCTGCAAACATTGGTCTGAAAAAACAAGGTTATTACCTGAGCGAGATTCAGGCAGA TGCTATTTTACGCATGAGCCTGCGAAACCTGACCGGCCTCGATCAGAAAGAAATTATCGAAAGCTACAAA AACCTGATGGGTAAAATCATCGACTTTGTGGATATCCTCTCCAAACCCGAACGCATTACCCAAATCATCC GTGACGAACTGGAAGAAATCAAAACCAACTATGGCGACGAACGCCGCAGCGAAATCAACCCGTTCGGCGG CGACATTGCCGATGAAGACCTGATTCCGCAACGCGAAATGGTCGTGACCCTGACCCACGGCGGCTATATA AAAACCCAGCCGACCACCGACTATCAGGCTCAGCGTCGCGGCGGGCGCGGCAAACAGGCGGCTGCCACCA AAGACGAAGACTTTATCGAAACCCTGTTTGTTGCCAACACGCATGACTATTTGATGTGTTTTACCAACCT CGGCAAGTGCCACTGGATTAAGGTTTACAAACTGCCCGAAGGCGGACGCAACAGCCGCGGCCGTCCGATT AACAACGTCATCCAGCTGGAAGAAGGCGAAAAAGTCAGCGCGATTCTGGCAGTACGCGAGTTTCCCGAAG ACCAATACGTCTTCTTCGCCACCGCGCAGGGAATGGTGAAAAAAGTCCAACTTTCCGCCTTTAAAAACGT CCGCGCCCAAGGCATTAAAGCCATCGCACTCAAAGAAGGCGACTACCTCGTCGGCGCTGCGCAAACAGGC GGTGCGGACGACATTATGTTGTTCTCCAACTTGGGCAAAGCCATCCGCTTCAACGAATACTGGGAAAAAT CCGGCAACGACGAAGCGGAAGATGCCGACATCGAAACCGAGATTTCAGACGACCTCGAAGACGAAACCGC CGACAACGAAAACACCCTGCCAAGCGGCAAAAACGGCGTGCGTCCGTCCGGTCGCGGCAGCGGCGGTTTG CGCGGTATGCGCCTGCCTGCCGACGGCAAAATCGTCAGCCTGATTACCTTCGCCCCTGAAACCGAAGAAA GCGGTTTGCAAGTTTTAACCGCCACCGCCAACGGATACGGAAAACGCACCCCGATTGCCGATTACAGCCG CAAAAACAAAGGCGGGCAAGGCAGTATTGCCATTAACACCGGCGAGCGCAACGGCGATTTGGTCGCCGCA ACCTTGGTCGGCGAAACCGACGATTTGATGCTGATTACCAGCGGCGGCGTGCTTATCCGTACCAAAGTCG AACAAATCCGCGAAACCGGCCGCGCCGCAGCAGGCGTGAAACTGATTAACTTGGACGAAGGCGAAACCTT GGTATCGCTGGAACGTGTTGCCGAAGACGAATCCGAACTCTCCGGCGCTTCTGTAATTTCCAATGTAACC GAACCGGAAGCCGAGAACTGA 


\section{In vitro growth measurements}

Mutant and parent bacteria were grown on solid media for 18 hours, and suspensions were made in gonococci broth media (GCB). Each bacterial suspension was passed through a 1.2 micron filter to remove aggregates, and the filtered suspensions were inoculated into $30 \mathrm{ml}$ of fresh GCB containing $100 \mu \mathrm{g} / \mathrm{ml}$ of streptomycin and supplemented with co-carboxylase, ferric nitrate, and $\mathrm{mM} \mathrm{NaH}_{2} \mathrm{CO}_{3}$ at the concentrations described (39). Cultures were adjusted to an initial optical density $\left(\mathrm{A}_{600}=0.075\right)$ and incubated under agitation at $37^{\circ} \mathrm{C}$. Absorbance recordings were taken each hour, and the average time needed to reach an $\mathrm{A}_{600}$ of 1.0 was established. All growth curves were conducted in triplicate.

For assessing relative in vitro fitness, competition co-cultures were performed with each mutant and corresponding parent strain. Similar numbers of mutant and parent bacteria were inoculated into supplemented GCB, starting with an $\mathrm{A}_{600}$ of approximately

0.08. Cultures were incubated under agitation at $37^{\circ} \mathrm{C}$ and $100 \mu \mathrm{l}$ aliquots were quantitatively cultured onto selective $(0.125 \mu \mathrm{g} / \mathrm{ml}$ ciprofloxacin $)$ and non-selective solid media at time zero to determine the input ratio. One hundred microliter aliquots were also collected and cultured in the same manner every 2 hours until an $A_{600}$ of 1.0 was achieved (approximately 8 hours) to determine the output ratios. A competitive index (CI) was established by dividing the ratio of mutant to parent CFU at each time point by the input ratio of mutant to parent CFU. CI values greater than 1 were interpreted as enhanced fitness of the mutant relative to parent; whereas, CI values less than 1 indicated reduced mutant fitness relative to parent. 


\section{Minimum Inhibitory Concentration (MIC) measurements}

The MICs of ciprofloxacin (Cip) and erythromycin (Em) were established for mutant and parent strain bacteria using a standard agar dilution assay (23). Reported ranges of intermediate level resistance and high level resistance to ciprofloxacin were used in accordance with CDC guidelines (23). The MIC of polymyxin B (PxB) was also determined using a standard agar dilution assay. PxB concentrations ranging from 200 to $900 \mu \mathrm{g} / \mathrm{ml}$ were used to establish MICs for strains JD4 and MS11.

\section{In vivo competitive infections}

Diestrus-stage female BALB/c mice (approximately 8 weeks old, National Cancer Institute, $\mathrm{NIH}$ ) and $c n l p^{-/-}$mice in the BALB/c background of a similar age (USUHS breeding colony) were administered water-soluble $17-\beta$-estradiol subcutaneously as described (80) to promote susceptibility to N. gonorrhoeae infection. Antibiotics (2.4 mg streptomycin and $0.4 \mathrm{mg}$ of vancomycin) were injected intraperitoneally twice each day and $0.04 \mathrm{~g}$ of trimethoprim sulfate per $100 \mathrm{ml}$ of drinking water was provided throughout the course of the experiment to minimize commensal flora. Mutant and parent $N$. gonorrhoeae strains were grown on solid media for 18 hours, and suspensions were passed through a 1.2 micron filter to minimize aggregates. Bacterial suspensions were adjusted to the same $A_{600}$ value and mixed in a 1 to 1 ratio. Twenty microliters of the mixed suspensions were inoculated intravaginally into mice and the inoculum was also quantitatively cultured on non-selective (total CFU) and selective $(0.125 \mu \mathrm{g} / \mathrm{ml}$ 
ciprofloxacin) (mutant CFU) solid media to establish input bacteria ratios. Vaginal swabs were quantitatively cultured on days $1,3,5$, and 7 post-inoculation similarly to determine the number of parent and mutant CFU recovered over time (output ratio). The competitive index was calculated for each mouse at each time point by dividing the number of mutant to parent CFU from each culture day (output ratio) by the number of input mutant to parent CFU (input ratio). CI values greater than 1 indicated enhanced fitness of the mutant relative to parent; whereas, CI values less than 1 indicated reduced mutant fitness relative to parent.

\section{RNA sequencing}

RNA sequencing was performed on N. gonorrhoeae strains FA19Sm ${ }^{\mathrm{R}}$, FA1090, MS11, AK1, JD1, and JD4. Total RNA was isolated using TRIzol (Invitrogen) from cells grown on solid media after 18 hour incubation at $37^{\circ} \mathrm{C}$. Ribosomal RNA (both $23 \mathrm{~S}$ and 16S) was depleted using a MICROBExpress ${ }^{\mathrm{TM}}$ Bacterial mRNA Enrichment Kit (Ambion) according to the manufacturer's instructions. Since approximately $80 \%$ of the total pool of RNA consists of rRNA and tRNA, this depletion step is necessary for enrichment of less abundant RNA species within the pool. Following RNA enrichment, first and second strand cDNA library generation was performed (Invitrogen) and all samples were qualified for purity $(260 / 280>1.7)$ and quantity (at least $10 \mu \mathrm{g}$ of total cDNA). All cDNA samples were sent to the Emory University Genomics Center for paired-end 100 base read deep sequencing using the Illumina platform. Raw data files 
were received from the Emory University Genomics Center, and data analysis was carried out using the CLC Bio Genomics Workbench suite.

\section{Deep sequencing data analysis}

The CLC Bio Genomics Workbench software suite was used for analysis of RNA sequencing data. Data from the imported files were normalized, and all sequence reads were mapped to the FA1090 published genome (the only annotated genome at the time). The length, count, and percent GC content for each read was captured, and coverage statistics were used to assess the quality of the coverage to reference genome. RNA sequencing analysis was selected for mapping to the FA1090 reference sequence with annotations. The Default software mapping settings were used for analysis. These mapping settings included a minimum length fraction of 0.9 , a minimum similarity fraction of 0.8 , and a maximum of 10 hits. The prokaryote setting was selected, and the

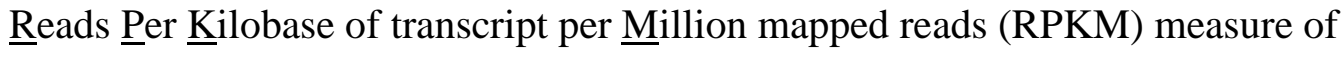
expression was used to address fold change differences in transcript profiles between mutant and parent strain. Scrutiny of global alterations in the transcriptome profile of mutant vs. parent gonococci included heat mapping and cluster analysis applications.

The Broad Institute’s GenePattern 2.0 software program was used for cluster analysis of RNA sequencing data. RPKM data was imported into GenePattern. Software filters were balanced for both upregulated and down-regulated genes. These filters included a 2.0 fold change cutoff and a p value $\leq 0.05$ (67). 


\section{Antimicrobial peptide susceptibility}

Assessment of bacterial susceptibility to cathelicidin-related antimicrobial peptide (CRAMP-38) was carried out in a microtiter plate assay. CRAMP-38 was synthesized by the Microchemical Facility of Emory University (75). Lyophilized CRAMP-38 was reconstituted to a concentration of $5 \mathrm{mg} / \mathrm{ml}$, and working stocks of 4, 8, 16, 32, and 64 $\mu \mathrm{g} / \mathrm{ml}$ were generated in a 1:2 dilution of GCB and $\mathrm{ddH}_{2} \mathrm{O}$ (dGCB). Bacterial strains to be tested were cultured on solid growth media. After 18 hrs incubation following an initial passage, bacterial suspensions were passed through a 1.2 micron filter to remove bacterial aggregates and diluted 1:500 in dGCB to an $\mathrm{A}_{600}$ of 0.075 . Approximately $10^{6}$ CFUs of each bacterial suspension was incubated with the appropriate concentration of CRAMP for a total volume of $100 \mu \mathrm{l}$ per well. Polypropylene microtiter plates were incubated for 55 minutes at $37^{\circ} \mathrm{C}$, and two 10 -fold serial dilutions were made in GCB containing $0.05 \%$ saponin. One hundred microliters from each well were cultured onto non-selective solid media and allowed to incubate for 24 hours. CFU were enumerated and results from each parent/mutant set and were compared. The number of CFU recovered from untreated controls was used to define 100\% recovery; the number of bacteria recovered from wells with CRAMP was expressed as a percentage of total recovery. 


\section{$\mathrm{H}_{2} \mathrm{O}_{2}$ sensitivity assay}

Sensitivity to hydrogen peroxide was conducted using an $\mathrm{H}_{2} \mathrm{O}_{2}$ saturated disk diffusion assay previously described (79). Briefly, $100 \mu$ l aliquots of bacterial suspensions of the mutant and parent strains were adjusted to an $\mathrm{A}_{600}$ of 0.1 and cultured on solid media in triplicate. Sterile paper disks saturated in one of three serially-diluted concentrations of $\mathrm{H}_{2} \mathrm{O}_{2}(1 \mu \mathrm{M}, 10 \mu \mathrm{M}$, and $10 \mathrm{mM})$ were placed onto solid GC plates. The diameter of the zone of growth inhibition around each disk was measured and recorded in millimeters following 24 hour incubation at $37^{\circ} \mathrm{C}$.

Sensitivity to $\mathrm{H}_{2} \mathrm{O}_{2}$ was also assessed using a modified version of a previously described liquid culture method (82). This method was chosen as a more quantitative measure of results obtained with the disk diffusion method. Briefly, mutant and parent gonococci were cultured to mid-log phase in liquid broth, diluted 1:10 in GCB, and subsequently split into separate cultures; one flask of GCB was cultured in the presence of $5 \mathrm{mM} \mathrm{H} \mathrm{H}_{2}$, while the other flask served as a negative control. Following a 15 minute shaking incubation at $37^{\circ} \mathrm{C}$, catalase was added to each $\mathrm{H}_{2} \mathrm{O}_{2}$-containing flask to a final concentration of $10 \mu \mathrm{g} / \mathrm{ml}$ followed by a 30 minute incubation to allow degradation of existing $\mathrm{H}_{2} \mathrm{O}_{2}$. Following the 30 minute incubation, $100 \mu$ l of each culture were quantitatively cultured onto solid GC media and allowed to incubate at $37^{\circ} \mathrm{C}$ for 24 hours. CFUs from each plate were enumerated, and the numbers of CFUs recovered for each mutant/parent set were compared. 


\section{Statistical analyses}

$\mathrm{H}_{2} \mathrm{O}_{2}$ disk diffusion measurements were recorded in triplicate and statistical significance was assessed for differences between the inhibition zone diameters around MS11 and JD4 using an unpaired $t$ test. To assess the statistical significance for any differences in the competitive index obtained in CRAMP knock-out mice relative to wild-type BALB/c mice, a one-sample $t$ test was performed on log-transformed data. The log transformation of the data was carried out to ensure the $p$ values obtained from the $t$ test were consistent with the geometric mean and confidence intervals.

\section{Animal Use Assurances}

Animal experiments were conducted in the laboratory animal facility at Uniformed Services University, which is fully accredited by the Association for the Assessment and Accreditation of Laboratory Animal Care, under a protocol approved by the University's Institutional Animal Care and Use Committee 


\section{CHAPTER 3: Results}

\section{A gyrA allele containing Ser91Phe and Asp95Asn substitutions confers intermediate level ciprofloxacin resistance to two other gonococcal strains}

We previously demonstrated that the $\operatorname{Cip}^{\mathrm{I}}$ gyr $_{91 / 95}$ resistant determinant in $N$.

gonorrhoeae (AK1) provides an in vivo fitness advantage when competed with the Cip ${ }^{\mathrm{S}}$ parent strain FA19 in the female mouse model of gonococcal genital tract infection. We moved this mutant allele into two other strains, FA1090 and MS11, to investigate the hypotheses that the elevated in vivo fitness associated with AK1 is not dependent on additional determinants and that mutant GyrA is sufficient to drive the observed fitness phenotype regardless of the strain background. The resultant mutants, JD1 and JD4, respectively, showed increased resistance to ciprofloxacin that fell within the Cip ${ }^{\mathrm{I}}$ range (Table 1). 
Table 3. Characteristics of the RNA sequencing analysis for each strain.

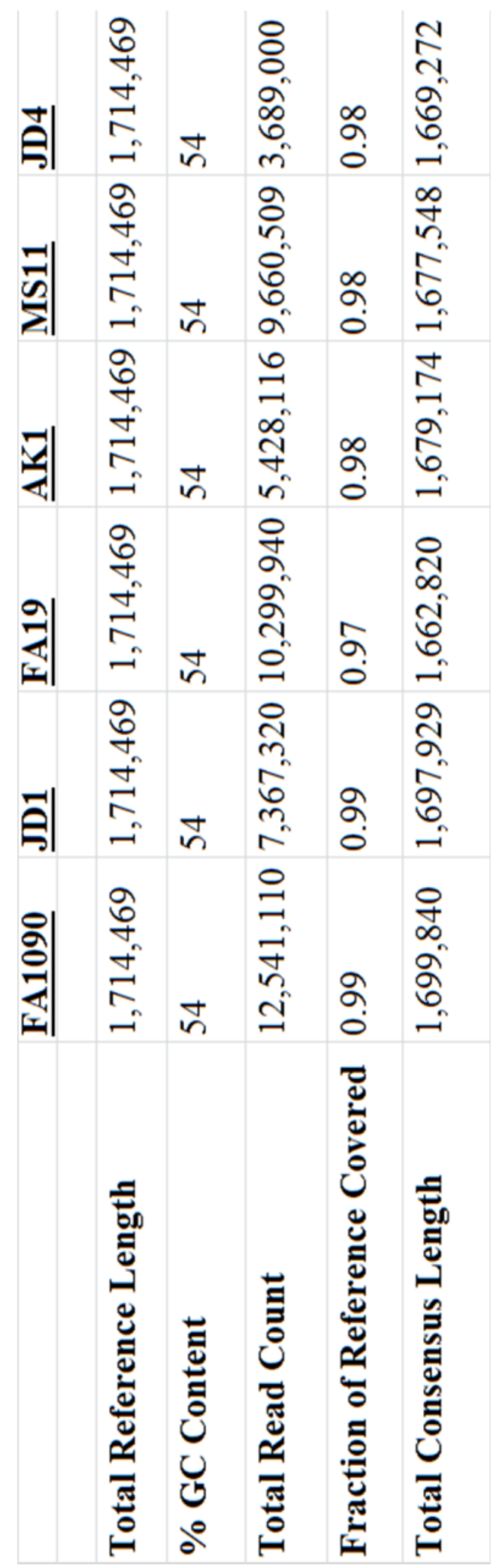




\section{Growth measurements and in vivo competitive infections}

The gyrA $_{91 / 95}$ mutants AK1, JD1, and JD4 displayed no significant difference in growth kinetics when cultured in liquid media compared to their isogenic parent strains (Fig 5). We also observed no difference in growth between JD1 and JD4 relative to that of their respective parent strains when co-cultured with their parent strains (Fig. 6).

Following the observation that gyr $A_{91 / 95}$ mutant AK1 exhibits an in vivo fitness advantage during experimental murine infection, we hypothesized that the resistance-conferring mutations in gyr $_{91 / 95}$ alone are sufficient to drive enhanced in vivo fitness of other gonococcal strains. Alternatively, the effect of the $g_{y} A_{91 / 95}$ mutations may be strainspecific as was reported for gyrA mutations in C. jejuni (51). Competitive murine infections were performed with each wild-type strain versus its respective $g_{y} A_{91 / 95}$ mutant. The results supported the hypothesis that the gyr $A_{91 / 95}$ allele is sufficient to drive an in vivo fitness advantage in different strain backgrounds. Interestingly, a difference in the degree of fitness advantage was observed with mutant JD4 displaying the most robust in vivo fitness advantage relative to parent strain MS11, followed by JD1 vs. FA1090 and AK1 vs. FA19 (Fig. 7) 
Figure 5. Growth kinetics of each gyrA $_{91 / 95}$ mutant versus its isogenic parent strain when cultured independently in liquid medium.

Growth was measured as change in absorbance at $600 \mathrm{~nm}$ over time. (A) FA19 versus AK1; (B) FA1090 versus JD1; (C) MS11 versus JD4. 
A

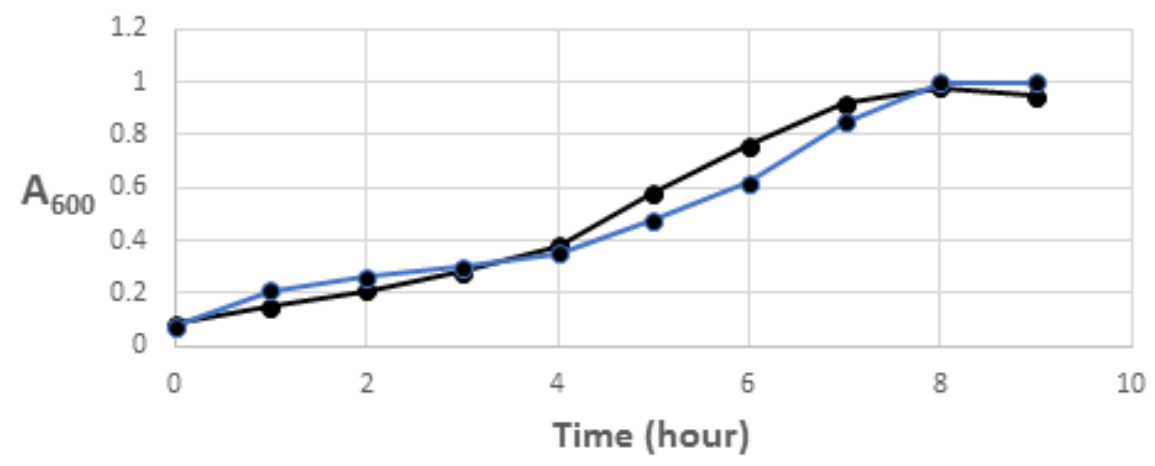

$\multimap$ FA19 $\longrightarrow$ AK1

B

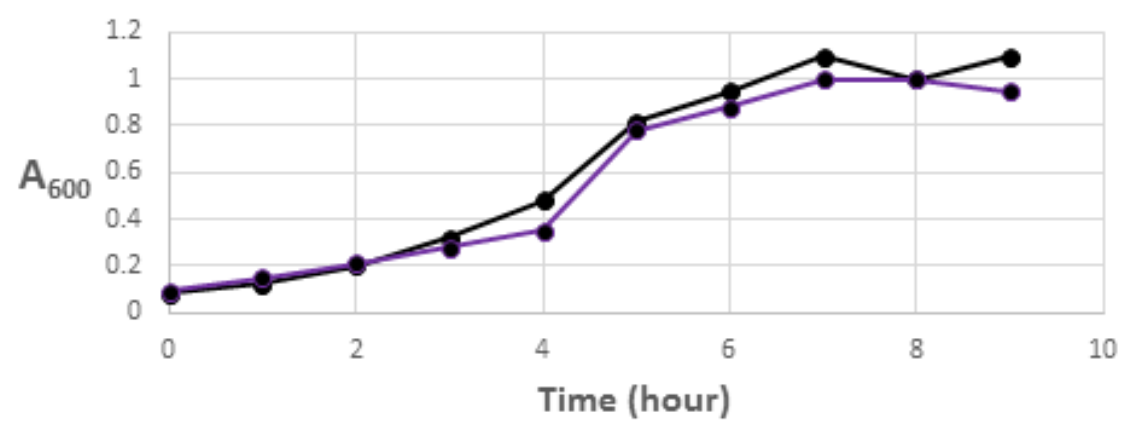

$\longrightarrow$ FA1090 ๑-JD1

C

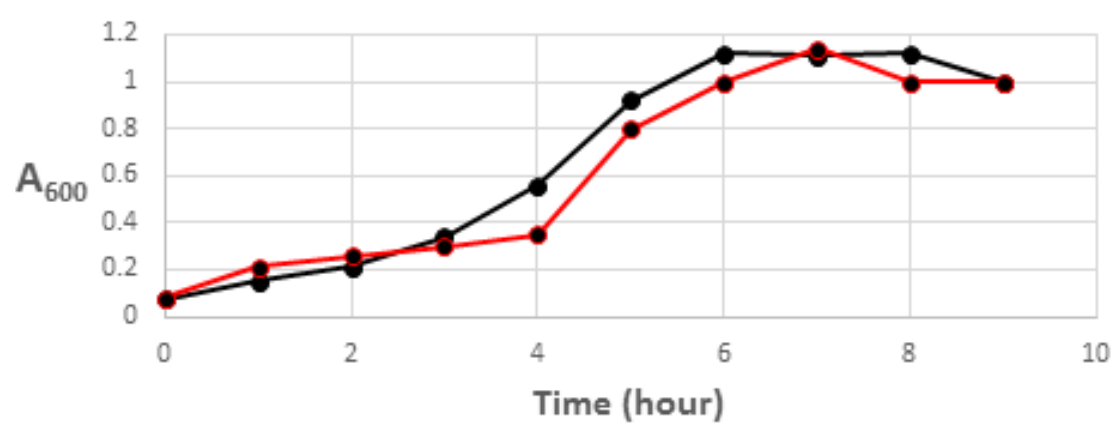

$\longrightarrow$ MS11 —JD4 
Figure 6. In vitro co-cultures of gyrA $A_{91 / 95}$ mutant and wild-type parent strains.

Data are shown as competitive indices (CI) measured at time 0 and early, midlogarithmic, late logarithmic and stationary phases. (A) FA1090 versus JD1; (B) MS11

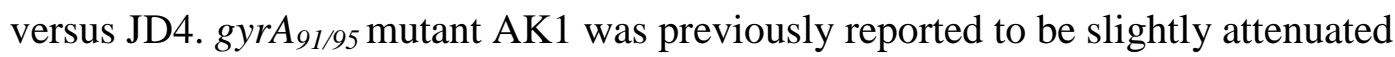
relative to FA19 during co-culture (47) and FA19 and AK1 were not re-tested here. 


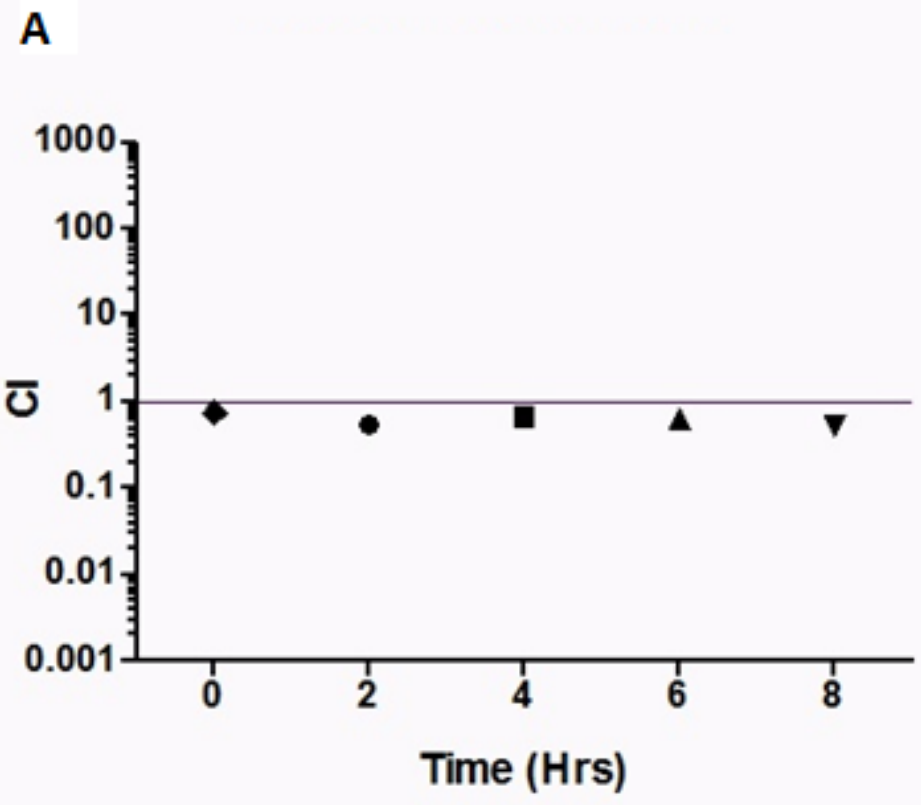

B

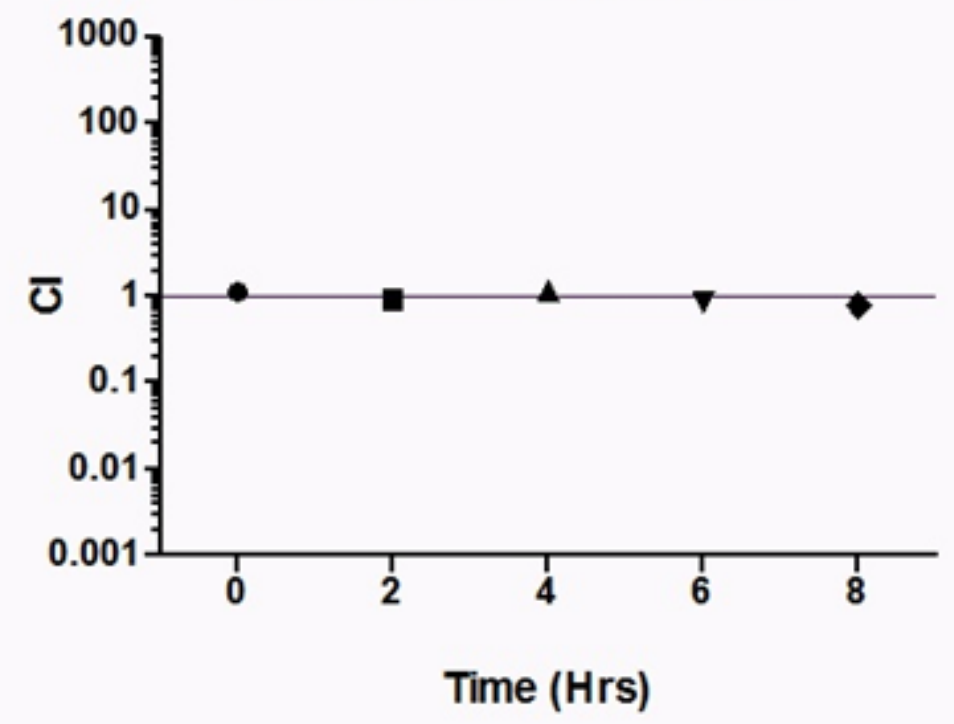


Figure 7. Three different gyr $_{91 / 95} \mathrm{Cip}^{\mathrm{I}}$ mutants out-compete their $\mathrm{Cip}^{\mathrm{S}}$ wild-type parents in an in vivo infection model.

The geometric means of the CIs (GM CI) of the combined data are indicated by the horizontal bar and stated below each graph. Open symbols represent vaginal cultures that yielded only mutant bacteria and the limit of detection (10 CFU) was used as the number of wild-type bacteria recovered for these data points. The experiment was performed twice with black and blue data points corresponding to the first and second experiment, respectively. 

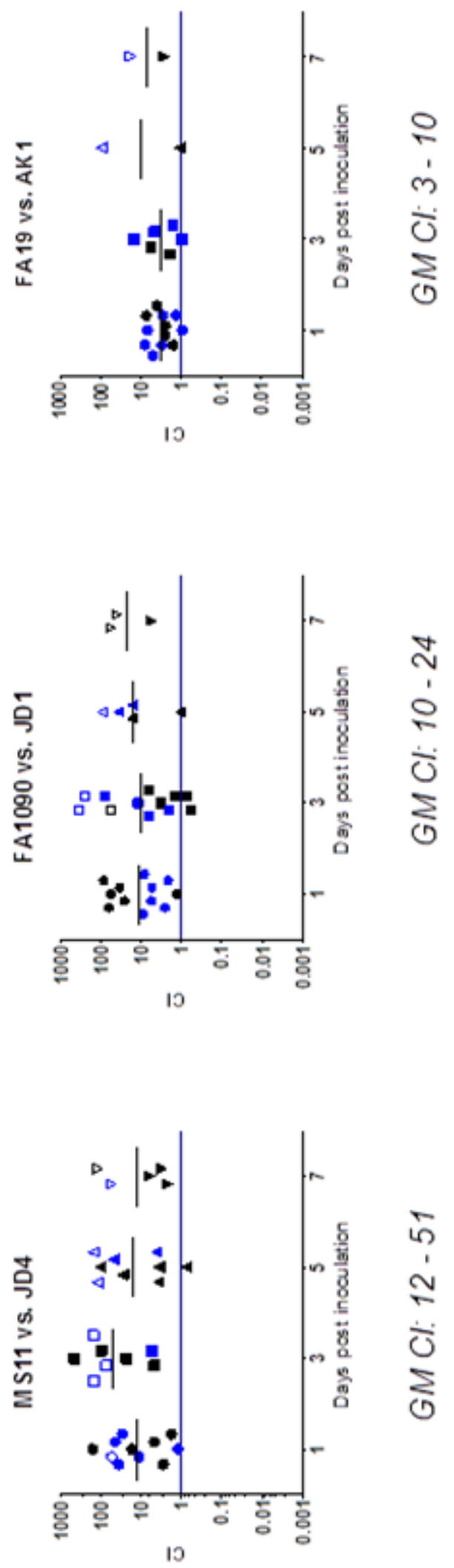


\section{Total RNA sequencing}

As mentioned previously, DNA gyrase helps manage genomic topology during DNA replication, transcription and translation through the introduction of negative supercoiling. DNA gyrase facilitates negative superhelicity, in part, to help relax the stress introduced through local unwinding of the duplex at the sites of transcription (90). Alteration of genomic supercoiling has been suggested to influence the expression of some genes to such an extent that regional supercoiling is considered a mechanism of gene regulation (62; 69). Considering this activity, we hypothesized that the double point mutations in gyrA that are responsible for conferring an intermediate-level resistance to ciprofloxacin recondition the genome of $N$. gonorrhoeae to cause changes in the transcriptome that promote survival in the context of the host environment.

As mentioned previously, FA1090 was used as a reference for all strains and mutants used in this experiment, and CLC Bio was used to generate total read counts and differential expression values for each pair. A summary of each profile is listed in Table 3. A heat map analysis of the data from the RNA sequencing analysis clearly showed conserved changes in gene expression patterns in bacteria carrying the mutated $g_{y} \mathrm{~A}_{91 / 95}$ allele relative to parent strains (Fig. 8), an observation which is consistent with the mutated GyrA molecule affecting gene transcription as hypothesized. However, this analysis was carried out using balanced filters for positive and negative fold changes. When the fold change cutoff is set at \pm 2 , our cluster analysis produces only positive fold change differences for mutants

A number of interesting gene candidates with differential expression profiles were tentatively identified in each parent and mutant set. Since the enhanced fitness phenotype 
was observed in vivo across three strains of $N$. gonorrhoeae, we chose to focus on transcript reads that were up-regulated ( $\geq 2$-fold above for at least two of the three mutant/wild-type pairs) or down-regulated ( $\geq 1$.5-fold below for at least two of the three mutant/wild type pairs) to identify genes that may be responsible for the observed in vivo phenotypes of the mutants. As shown in Fig. 5 a negative 2-fold change cutoff failed to produce down-regulated mutant genes; therefore, a decision was made to include a 1.5 fold decrease in mutant transcript reads to help enrich for relevant biological changes resulting from gene down-regulation. The total number of RNA sequence reads for parent strains was higher than that of mutant bacteria; because of the lower total mutant read count, we aimed for a negative fold change absolute value that was slightly lower (1.5) than the value set for the positive fold change cut-off (2). We acknowledge that many interesting genes may not have been captured as a result of these selection criteria; however, our goal was to identify phenotypically relevant transcriptional changes in an initial analysis with expanded data analysis planned for future studies.

These selection criteria produced a collection of 72 up-regulated genes in the mutant bacteria compared to their isogenic parent strains. In an effort to isolate a network or pathway of genes that might be responsible for enhanced in vivo fitness, annotated genes that were differentially altered in each set were grouped into five categories. These categories included genes with metabolic functions, genes encoding surface-expressed proteins, genes encoding transport-associated proteins, regulatory genes, and genes involved in iron uptake and utilization (Tables 4, 5, 6 and 7). A total of 26 genes were down-regulated; however, these reads were not assigned to a particular category (Table 8). 
Many of the mutant sequence reads displaying the largest differential relative to isogenic parent bacteria had minimal or no annotation in the National Center for Biotechnology Information database, and only a putative functional domain existed for these candidates at the time of analysis. Up-regulated genes were selected based on the rationale that increased production a specific gene product could be responsible for promoting fitness within resource-limited environment of the host. For instance, expression of NGO2093 (a putative ferric receptor) was elevated greater than 2.5 fold in both JD4 and JD1 and nearly 2 fold in AK1 (Table 7). While iron availability is plentiful under in vitro culture conditions, sources of adequate iron during infection require bacterial scavenging of host-derived iron. Increased expression of surface factors that facilitate acquisition of host iron may provide a competitive edge for mutant bacteria over parent strains. Increased expression of genes involved in DNA damage and repair (Table 6) may offer mutant bacteria a means of sustained survival during oxidative stress.

Differential expression of genes involved in the biosynthesis of thiamine emerged as particularly interesting candidates responsible for enhanced fitness because thiamine, an essential vitamin or growth cofactor, is not produced by the host and therefore, increased production of thiamine in the mutants might confer a growth advantage during infection (Table 4). With a focus on the role that elevated thiamine biosynthesis potentially may contribute to in vivo fitness, we explored the hypothesis that mutant bacteria would outcompete parent strains in vitro in the absence of media supplementation with saturating thiamine. 
Table 4. Up-regulated genes associated with the biosynthesis of thiamine.

\begin{tabular}{|c|c|c|c|c|}
\hline Gene & Protein name or predicted function & JD4/M S11 & JD1/FA1090 & AK1/FA19 \\
\hline NG02041 & ThiC & 6.67 & 2.99 & 1 \\
\hline NGO2003 & Thiazole biosynthesis & 2.62 & 2.23 & 1.45 \\
\hline NGO2006 & This & 5.67 & 3.13 & 0.81 \\
\hline NGO2007 & Thiamine-phosphate phosphorylase (ThiE) & 2.89 & 4.26 & 1.58 \\
\hline NGO2005 & ThiG & 5.06 & 2.38 & 2.03 \\
\hline NGO2008 & Oxidoreductase & 6.02 & 3.15 & 1.76 \\
\hline NGO2009 & Permease (sodium:solute symporter family) & 2.34 & 2.38 & 1.06 \\
\hline
\end{tabular}


Table 5. Up-regulated putative genes involved in gene regulation

\begin{tabular}{|c|c|c|c|c|}
\hline Gene & Protein name or predicted function & $\underline{\text { JD4/MS11 }}$ & $\underline{\text { JD1/FA1090 }}$ & $\underline{\text { AK1/FA19 }}$ \\
\hline NGO0025 & AraC family transcriptional regulator & 6.16 & 2.65 & 1.89 \\
\hline nrdR & Transcriptional regulator & 3.71 & 2.39 & 1.27 \\
\hline NGO0727 & putative regulatory protein, gntR family & 9.6 & 2.03 & 1.84 \\
\hline
\end{tabular}


Table 6. Up-regulated genes associated with general metabolism and DNA damage and repair.

\begin{tabular}{cl} 
Gene & \multicolumn{1}{c}{ Protein name or predicted function } \\
NG01521 & Predicted acetate kinase \\
NG00806 & TCysN_NoDQ_II: \\
NG00087 & glycosyltransferase \\
NG00031 & Acetyltransferase; NAT (N-Acyltransferase) \\
NG02008 & Oxidoreductase \\
NG00886 & acyl-CoA dehydrogenase: \\
NG00886 & Acyl-CoA dehydrogenase \\
NG01089 & Possible arginine decarboxylase \\
NG01091 & Predicted binding partner of NGO1089 \\
NG00499 & Putative ubiquinol-cytochrome C reductase \\
NG00501 & Predicted association with NGO0499 \\
NG00502 & Predicted association with NGO499 \\
NG00555 & Putative Zn-dependent protease \\
NG01094 & Putative Zn metallopeptidases \\
NG01093 & Putative PE family \\
NG00518 & NIpC/P60 superfamily \\
NG01733 & YjeQ \\
NG00083 & UDP-Glcnac inverting 4,6-dehydratase \\
NG00026 & Prolyl endopeptidase \\
NG00076 & Esterases \\
NG00234 & Coproporphyrinogen III oxidase \\
NG00084 & PglC \\
NG01020 & Putative integrase \\
NG01136 & Glycosyltransferase \\
NG01739 & Putative type II restriction endonuclease \\
NG02047 & FAD binding domain \\
NG02068 & Zinc metal lopeptidase \\
NG02050 & Imelysin peptidase \\
\hline NG & \\
NG
\end{tabular}

$\underline{\text { JD4/MS11 JD1/FA1090 AK1/FA19 }}$

\begin{tabular}{ccc}
8.73 & 3.21 & 2.71 \\
5.63 & 3.68 & 3.29 \\
2.62 & 2.05 & 1.78 \\
2.17 & 2.02 & 1.96 \\
6.02 & 3.15 & 1.76 \\
2.31 & 2.73 & 1.53 \\
2.31 & 2.73 & 1.53 \\
2.16 & 2.58 & 4.95 \\
2.01 & 4.63 & 3.16 \\
5 & 4.6 & 6.19 \\
4.36 & 2.87 & 9.22 \\
20.43 & 2.67 & 5.67 \\
1.5 & 4.38 & 2.41 \\
4.25 & 3.66 & 2.34 \\
5.47 & 4.33 & 2.69 \\
1.71 & 3.78 & 2.69 \\
2.43 & 3.39 & 1.73 \\
3.05 & 3.29 & 1.78 \\
1.41 & 3.13 & 2.01 \\
5.99 & 1.92 & 3.48 \\
2.11 & 2.06 & 1.57 \\
2.95 & 2.26 & 1.69 \\
2.39 & 1.62 & 2.27 \\
2.62 & 5.11 & 1 \\
8.73 & 2.83 & 1.08 \\
2.27 & 2.27 & 1.04 \\
3.09 & 2.09 & 1.23 \\
2.53 & 2.81 & 1.08 \\
& & \\
\hline
\end{tabular}


Table 7. Up-regulated genes associated with iron utilization or transport.

Gene

NGO0023

NGO2093

NGO2092

NGO2011

NGO0500

NGO2091

NGO2009

NGO2090

NGO0498

NGO1097

NGO2097

NGO2092

NGO0023

NGO1497

\section{Protein name or predicted function}

$\mathrm{ABC}$ transporter periplasmic binding protein

Fet A (ferric receptor)

Ferric periplasmic binding protein

Amino acid ABC transporter permease

Homology to Protein gp6; possible adhesion domain

ABC transporter; Transmembrane subunit

Permease (sodium:solute symporter family)

$\mathrm{ABC}$ transcporter permease

Pertactin-like passenger domains

Homology to FimV, Tfp pilus assembly

Prokaryotic membrane lipoprotein lipid attachment

Ferric enterobactin periplasmic binding protein

ABC transporter periplasmic binding protein

Transferrin receptor for transferrin utilization

\section{$\underline{\text { JD4/MS11 }} \underline{\text { JD1/FA1090 }}$ AK1/FA19}

3.51

2.63

2.7

2.92

1.66

3.08

3.83

3.4

3.33

2.38

2.67

2.97

2.02

2.27

3.08

2.7

6.81

1.78

1.99

2

1.39

4.29

1.49

1.06

2.06

4.31

2.58

1.34

2

3.51

5.2
1.78

No change 
Table 8. Down-regulated genes.

Gene

Predicted Function

NGO0589 Uracil-xanthine permease (ncs2)

NGO0201 IS1016 transposase

NGO0847 Predicted metal-binding protein related SecA

NG01435 CadB

NGO0631 Predicted functional association with GyrA

NG00629 GyrA

NG01671 Dephospho-coenzyme A kinase (DPCK)

NGO1054 Predicted functional association with RecX

\section{$\underline{\text { JD4/MS11 }} \underline{\text { JD1/FA1090 }}$ AK1/FA19}

$-2.13$

$-2.13$

$-1.39$

$-2.03$

$-1.59$

$-1.07$

$-2.01$

$-1.56$

1.05

$-2.01$

$-1.24$

$-1.59$

$-1.62$

$-3.05$

$-1.34$

$-1.54$

$-2.19$

$-1.11$

$-1.51$

$-2.77$

$-1.54$

$-1.24$

$-2.55$

$-1.63$ 
Figure 8. Heat signatures for comparative transcriptome expression profiles.

Parent strain vs. mutant heat maps. The color scale differentiates lower levels of normalized transcript read counts (blue) from higher levels (red). The cluster shown reflects commonly up-regulated mutant genes relative to all three parent strains. 


\section{Description}

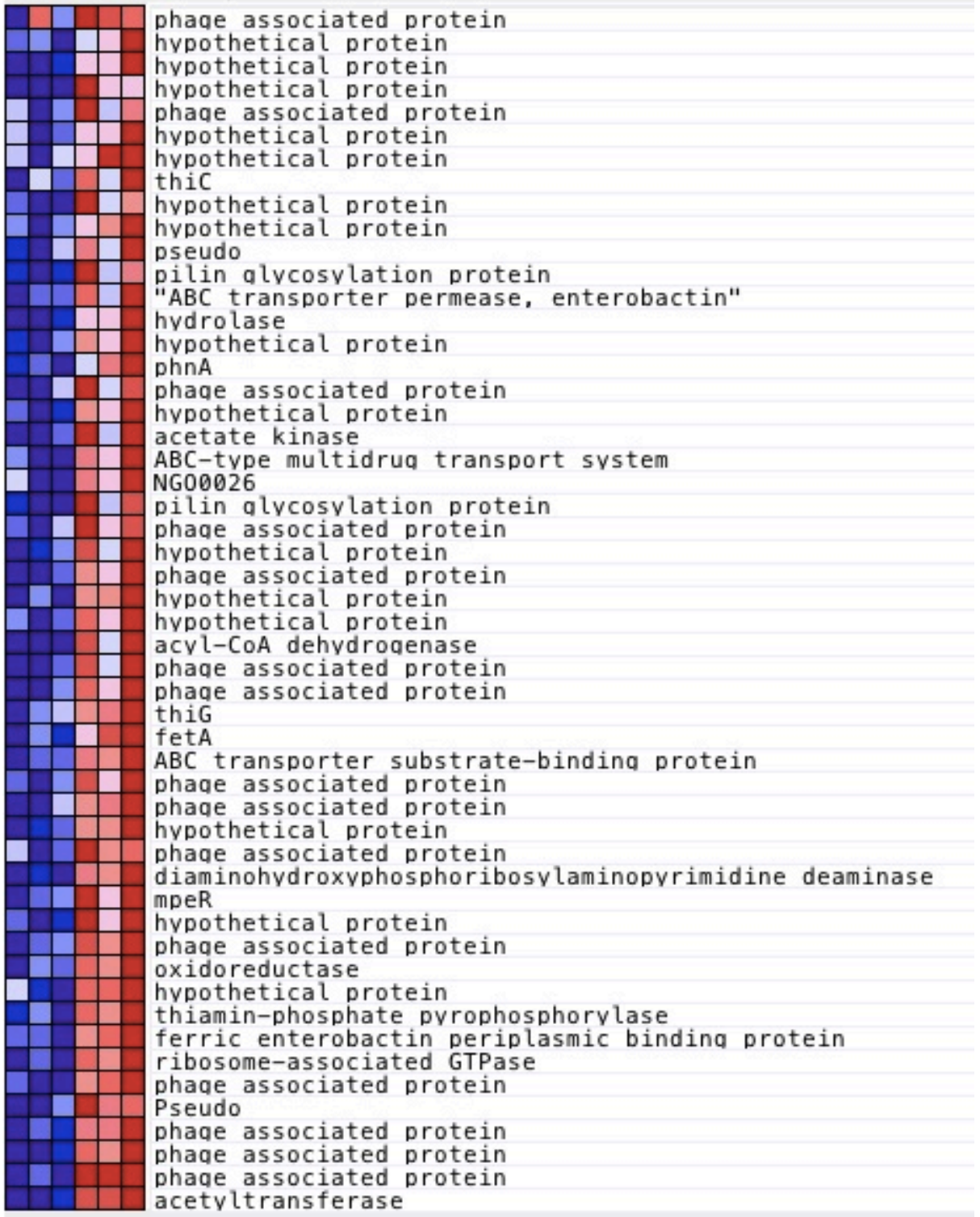




\section{In vitro growth in the absence of co-carboxylase}

In the early 1940's, studies exploring the ideal in vitro growth conditions for $N$. gonorrhoeae identified a need to supplement culture media with co-carboxylase to be able to isolate occasional strains that were shown to be thiamine auxotrophs (48). The terminal product in the thiamine biosynthesis pathway is thiamine pyrophosphate; cocarboxylase is the common name. We hypothesized that the possible increased bioproduction of thiamine in mutant bacteria, as suggested by the RNA sequencing data, would provide an in vitro growth advantage relative to parent strains when cultured in media without supplemented thiamine. However, mutant and parent $N$. gonorrhoeae grown independently or as co-cultures in liquid media not containing co-carboxylase supplementation displayed no difference in growth characteristics (data not shown). We therefore did not pursue this hypothesis further. We also considered the possibility that one of the genes shown to be up-regulated in our dataset (NGO2008) is an oxidoreductase that may provide decreased susceptibility to antimicrobial peptides (65).

Attempts to validate the elevated expression of selected transcripts that appeared to be differentially regulated (i.e. NGO 2008, thiC, and thiG) using quantitative real-time PCR were unsuccessful. It is possible that the low copy number of these transcripts limited the utility of quantitative PCR as a tool for validation of these candidates. Therefore, we directed our efforts towards in vitro assays designed to measure gonococcal resistance to host innate defenses. 


\section{Sensitivity to $\mathrm{H}_{2} \mathrm{O}_{2}$}

Hydrogen peroxide disk diffusion assays were used as a surrogate assessment of

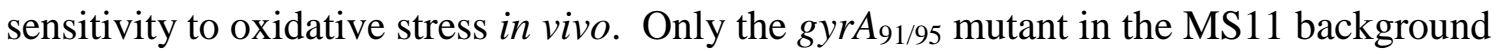
(mutant JD4) displayed a significantly smaller zone of inhibition around $\mathrm{H}_{2} \mathrm{O}_{2}$-saturated filter disks compared to the parent strain (Fig. 9). In an attempt to better quantify the sensitivity of the strains to $\mathrm{H}_{2} \mathrm{O}_{2}$, a broth culture method was also used. Bacteria were exposed to $\mathrm{H}_{2} \mathrm{O}_{2}$ in liquid culture under agitation in duplicate experiments, but none of the three mutants showed any difference in susceptibility to $\mathrm{H}_{2} \mathrm{O}_{2}$ relative to the respective parent bacteria.

\section{Sensitivity to cationic antimicrobial peptides}

Several cationic antimicrobial peptides are inducibly expressed by epithelial cells in the female genital tract, including the cathelicidins. These innate effectors are also carried within phagocytic granules. The gyrA $_{91 / 95}$ mutant JD4 was also more resistant to cathelicidin-related antimicrobial peptide (CRAMP), the murine homolog of the human cathelicidin LL-37, compared to parent strain MS11 as evidenced by a significantly higher effective concentration required for killing $50 \%$ of the bacterial population $\left(\mathrm{EC}_{50}\right)$

value (Fig. 10). This observed increase in CRAMP tolerance was supported with duplicate measurements of the minimum inhibitory concentration (MIC) of polymyxin B $(\mathrm{PxB})$, which is a bacterial cationic antimicrobial peptides that is often used in in vitro studies. Agar dilution assays yielded a PxB MIC of $850 \mu \mathrm{g} / \mathrm{ml}$ against JD4, while the 
PxB MIC for MS11 was $500 \mu \mathrm{g} / \mathrm{ml}$. In contrast, and similar to results from $\mathrm{H}_{2} \mathrm{O}_{2}$ assays, the presence of the gyrA $_{91 / 95}$ mutations in the FA19 or FA1090 background did not confer statistically significant differences in susceptibility to CRAMP and PxB MIC were similar for mutant and parent strains.

\section{In vivo competitive infection in wild-type and $C R A M P^{-/-} B A L B / c$ mice}

For an in vivo assessment of the susceptibility of MS11 and mutant JD4 to host antimicrobial peptides, we carried out parallel in vivo competition assays in wild-type BALB/c mice and BALB/c mice that are genetically deficient in the production of CRAMP. We predicted an ablation of the in vivo fitness advantage shown by JD4 when competed against MS11 in CRAMP-deficient BALB/c mice. Groups of wild-type and CRAMP-deficient mice were inoculated with mixed suspensions containing similar numbers of MS11 and JD4 bacteria, and the relative ratio of mutant to wild-type bacteria recovered from mice relative to that of the inoculum was determined as described in the Materials and Methods. In both sets of mice, mutant JD4 displayed a fitness advantage over MS11within one day of infection, with pure cultures of gyrA $_{91 / 95}$ mutant bacteria isolated from two mice at this time point (open circles) (Figure 10). On days 3 and 5, a 6 and 18 fold increase in fitness was observed for the mutant in normal BALB/c mice. In contrast, no fitness advantage was observed for JD4 in the CRAMP-deficient BALB/C mice. Comparison of the CIs obtained from wild-type and CRAMP-deficient mice on days 3 and 5 showed a significant difference at the level of $p=0.02$ and $p=0.013$ respectively. We conclude that the ciprofloxacin resistance-conferring gyr $_{91 / 95}$ mutant of 
strain MS11 demonstrates enhanced fitness in a surrogate model of female genital tract infection due to reduced susceptibility to CRAMP. 
Figure 9 The gyrA $_{91 / 95}$ mutant in the MS11 background displayed decreased sensitivity to hydrogen peroxide.

Statistical significance was measured using an unpaired $t$ test on mutant and parent inhibition zone diameters. 


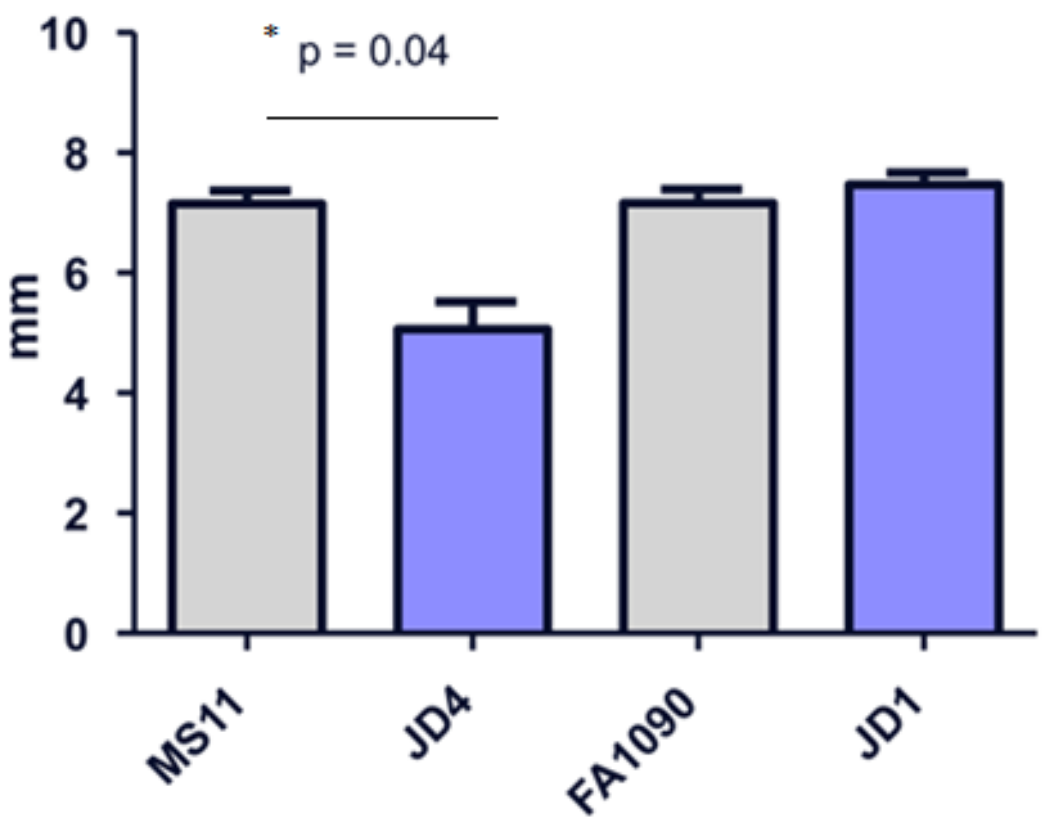


Figure 10. The gyrA $_{91 / 95}$ mutant in the MS11 background displayed decreased sensitivity to CRAMP.

The effective concentration of peptide required to kill 50\% of the bacterial population $\left(\mathrm{EC}_{50}\right)$ for mutant and parent bacteria was determined in duplicate. CFUs were enumerated from the $10^{-5}$ dilution series, and each parent/mutant set was compared. The total CFU count from each untreated control was used to determine $100 \%$ recovery, and the CFUs recovered for each strain at each concentration of CRAMP tested was reflected as a percentage of total recovery. An $\mathrm{EC}_{50}$ value was extrapolated from the $\mathrm{EC}_{50}$ shift curve, and statistical significance was determined using an unpaired $t$-test. Only JD4 displayed a statistically significant increase in the CRAMP concentration required for an $\mathrm{EC}_{50}$. 


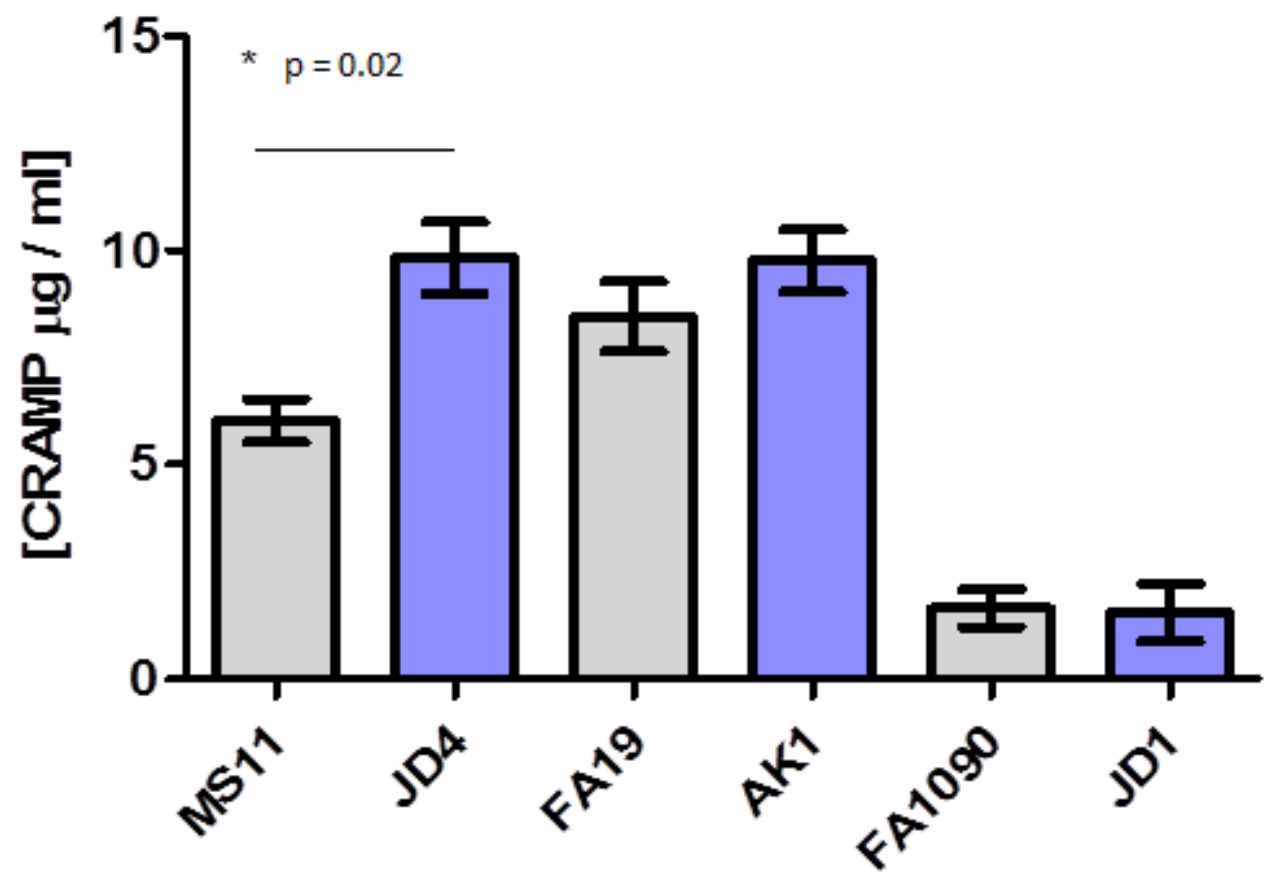


Figure 11. Competitive infections in CRAMP-sufficient (wild-type) and CRAMPdeficient BALB/c mice.

In vivo competitive infections competing gyrA91/95 mutant JD4 against MS11 were conducted in parallel. The geometric mean CIs are shown as horizontal bars. Black symbols represent bacteria cultured from wild-type BALB/c mice, and red symbols represent bacteria cultured from CRAMP deficient mice. Open symbols represent vaginal cultures that yielded only mutant bacteria and the limit of detection (10 CFU) was used as the number of wild-type bacteria recovered for these data points. The experiment was performed twice and the combined data are shown. Comparison of the log-transformed data from days 3 and 5 showed a significant difference as indicated (unpaired $t$ test). 
Day 1 Day 3 Day 5

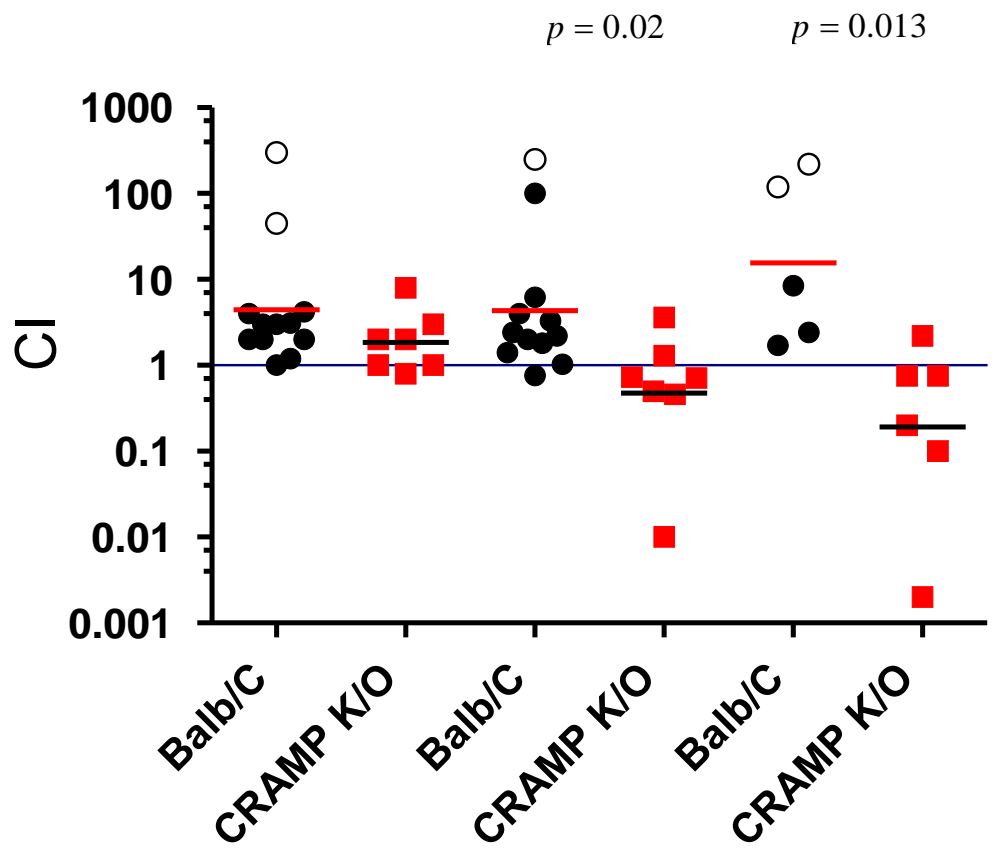




\section{CHAPTER 4: Discussion and Future Directions}

There has been a steady rise of antibiotic resistance in Neisseria gonorrhoeae against several classes of antibiotics beginning with the introduction of sulfonamide therapy in the early 1940’s. The loss of fluoroquinolone in 2007, and the more recent loss of the extended spectrum cephalosporins used as single therapies, has further complicated management of gonococcal infections. Current antibiotic treatment for gonorrhea is recommended to include a combined approach of ceftriaxone and azithromycin.

The era of fluoroquinolone treatment against gonorrhea exemplifies an accelerated evolutionary path to acquisition of resistance with less than 15-20 years between the introduction of fluoroquinolones against gonorrhea and the spread of an unacceptable percentage of resistant strains. The discovery that a commonly identified gyrA allele that confers intermediate level resistance to fluoroquinolones in $N$. gonorrhoeae also promotes in vivo fitness may help explain the short duration of the effectiveness of fluoroquinolone therapy. As previously mentioned, the acquisition of fluoroquinolone resistance in $N$. gonorrhoeae occurs through a step-wise process, where certain double point mutations in the gyrA gene produce two key amino acid changes at positions 91 and 95, which confers an intermediate level of resistance. An additional point mutation in the parC gene at position 86 confers high level resistance. While it appears that acquisition of both gyrA and parC mutations provides an unfavorable fitness cost in vivo, we hypothesize that the fitness advantage associated with $N$. gonorrhoeae harboring only the gyrA $_{91 / 95}$ resistance-conferring mutations facilitates the path to 
fluoroquinolone resistance. It is important to note that enhanced fitness was not observed with in vitro co-culture experiments.

As was recently reported for strain FA19, here we showed that gyrA91/95 mutations that confer intermediate-level FQ resistance result in enhanced in vivo fitness of $N$. gonorrhoeae in two other strain backgrounds, and the fitness advantage was greater in strains FA1090 and MS11 compared to strain FA19. This finding is different than that reported for gyrA mutants in C. jejuni, which only increased in vivo fitness in some Campylobacter strains (19). Since the introduction of gyrA $\mathrm{A}_{91 / 95}$ alone into three unrelated strains of N. gonorrhoeae is sufficient to promote fitness, we conclude that the influence of mutant gyrA alleles on the propagation and maintenance of antibiotic resistant $N$. gonorrhoeae may be stronger than that observed in C. jejuni.

Considering the ability of type II topoisomerases to provide broad regulation of the bacterial genome, we hypothesized that the fitness advantage associated with gyr $_{91 / 95}$ mutations may be a consequence of an altered transcriptional profile, which could result in bacteria that are better suited for survival during genital tract infection. Differences were observed between three sets of mutant and parent transcriptomes, which supports our hypothesis and claims that resistance-conferring mutations in GyrA affect global levels of transcription in other Gram-negative bacteria (21). Although we categorized up-regulated and down-regulated genes with respect to phenotypes that may alter gonococcal fitness in an attempt to capture a molecular mechanism linked to our in vivo observations, we do not yet know which, if any of these differences are responsible for the observed gyrA $_{91 / 95}$-driven fitness advantage. In the context of infection, the resource-limited environment of the host pressures the ability of bacteria to synthesize or 
scavenge essential nutrients or metabolites; additionally, increased expression of surface factors involved in evasion of innate defenses or adherence to host cells may be selected in vivo. Accordingly, genes we identified fall into those categories that may provide mutant bacteria with an advantage over parent, such as metabolism or iron uptake.

The use of in vitro grown rather than in vivo grown bacteria as the source of RNA for deep sequencing and the fact that the assay was done only once are two limitations to the RNA sequencing approach we utilized. While we see global changes in the transcriptome of the gyrA mutants relative to parent strains, capturing biologically relevant transcriptional changes that confer enhanced in vivo fitness may require bacterial growth within the host. Rather than sequencing RNA pools from bacteria grown in vitro, one could inoculate BALB/c mice with pure mutant or parent bacteria in triplicate, and on the day we typically see the largest competitive index (for example, day 3 from Fig. 2), we could inoculate a plate with vaginal material for culture and also transfer the vaginal swab directly into TRIzol@ for immediate RNA extraction. Parallel sequencing of RNA from both the cultured $N$. gonorrhoeae as well as directly from the swab material, followed by subtraction of reads that do not map to the reference or cultured sequence should provide host-directed transcriptional profiles. There was not wide acceptance of this technique for defining the transcriptome of bacteria sampled directly from an infected host when we started this work; since the completion of this study in 2012, several methods have been employed to investigate host-directed RNA levels in bacteria $(16 ; 29 ; 31 ; 42 ; 84 ; 99)$

Considering the role that unidentified non-coding small RNAs may play in the regulation of key genes, a third limitation of the RNA seq analysis we performed is that 
transcriptional differences in small RNAs might not have been detected. Key alterations may be limited by the degree of small non-coding RNA copy numbers, and potential quantitative differences in these regulatory RNAs may result in divergent protein expression between JD4 and MS11 for key genes that display relatively equal rates of transcription. Therefore, expanding subsequent rounds of deep sequencing to include an analysis of differences in small RNA levels would help broaden the spectrum of genes influenced by mutant GyrA.

Symptomatic gonorrhea infections present with a characteristic PMN influx at the site of infection. PMNs kill microbes through the production of reactive oxygen species and the release of granular contents, which include cationic antimicrobial peptides. Hostderived cationic antimicrobial peptides are also produced by epithelial cells and thus provide another barrier to bacterial colonization. We utilized in vitro assays to assess these challenges in an attempt to explain the fitness advantage associated with GyrA mutant bacteria. We found that the gyrA $_{91 / 95}$ mutant of strain MS11 was less susceptible to oxidative stress and antimicrobial peptides than the parent strain; however, this difference was not observed in the gyrA $_{91 / 95}$ mutants of FA19 or FA1090, although the concentration of CRAMP used against FA1090 may have been too high to detect a difference. While we have shown in vitro data suggesting that JD4 is less susceptible to $\mathrm{H}_{2} \mathrm{O}_{2}$ than MS11, we currently cannot further support the hypothesis that decreased susceptibility to oxidative stress contributes to enhanced in vivo fitness. PMN killing assays might support an in vivo role for the observed decrease in $\mathrm{H}_{2} \mathrm{O}_{2}$ susceptibility by JD4, although thus far, no known anti-oxidant factors, including catalase and cytochrome 
C peroxidase produced by $N$. gonorrhoeae have been shown to protect the gonococcus from PMN killing in vitro or in NADPH-oxidase defective mice (39; 79; 103).

In contrast to our inability to comment on the relevance of $\mathrm{H}_{2} \mathrm{O}_{2}$ sensitivity in vivo, studies in CRAMP-deficient mice support gyr $_{91 / 95}$-driven changes in resistance to CRAMP as the reason for the in vivo fitness advantage of mutant JD4. The mechanism by which $g y r A_{91 / 95}$ mutations confer increased resistance to antimicrobial peptides in strain MS11 is not known. We were surprised by the data reflecting this difference between JD4 and MS11, since MS11 is already highly resistant to cationic antimicrobial peptides due to a mutation that causes de-repression of the $m \operatorname{tr} C D E$ active efflux pump and a second mutation that generates an MtrR independent promoter (60; 98). Overexpression of the mtrCDE operon is one mechanism by which gonococci protect against antimicrobial peptides, but transcription of $m \operatorname{tr} C$ or $m t r A$, the pump activator, was not increased in mutant JD4 relative to the wild-type parent strain in the RNA seq analysis. However, it is still possible that the decreased sensitivity to CRAMP and polymyxin B exhibited by mutant JD4 may be the result of accelerated efflux through the MtrCDE system. Although the RNA sequencing data did not suggest altered expression of these pumps in the mutant bacteria, future attempts to assess transcriptional differences in lptA or mtrCDE using RT-PCR may yield different results. Additionally, one might predict that JD4 would be more resistant to other substrates of these efflux pumps such as progesterone and bile salts if efflux was increased. These possibilities should be explored.

Other mechanisms by which gonococci evade antimicrobial peptides is through changes in surface structures such as decoration of lipid A with phosphoethanolamine 
(49) and sialylation of the N-neotetraose moiety of the LOS chain (Wu H and A E Jerse, in preparation). Both of these mechanisms are fully operable in MS11, and neither the phosphoethanolamine transferase (lptA) gene or sialyltranferase (lst) gene were upregulated in mutant JD4 compared to the wild type strain. It is possible that lptA or lst expression is increased in the gyrA mutant bacteria indirectly through the altered expression of a non-coding small RNA that has not yet been identified. Alternatively, it is possible that the stability of LptA is enhanced in the gyrA mutants due to an elevated expression of oxidoreductase, which introduces stabilizing disulfide bonds to periplasmic proteins. Neisseria spp. express three oxidoreductases, and inactivation of each one these has been shown to increase sensitivity to antimicrobial peptides in $N$. meningitidis (66). Several genes with domains predicted to function as an oxidoreductase were shown to be elevated in the RNA sequencing data set (Table 4 and 6). The oxidoreductase with the greatest fold change difference relative to parental strains (NGO2008) was investigated for a potential role in increased resistance to polymyxin b. However, disrupting the NGO2008 open reading frame with a chloramphenicol-resistance cassette in both JD4 and MS11 did not alter the MIC to polymyxin b relative to unaltered mutant and parent bacteria. Furthermore, initial attempts to validate the NGO2008 transcriptional differences observed between mutant and parent in the RNA sequencing data with quantitative real-time PCR failed to suggest a difference in these transcript levels, albeit the RNA sequencing needs to be repeated and analyzed with more scrutiny.

It is interesting that $g y r A_{91 / 95}$ promotes in vivo fitness in all three strains of $N$. gonorrhoeae tested, but the mechanism responsible for this phenotype appears to be strain specific. This finding suggests that transcriptional changes directed by mutant 
GyrA molecules can be tailored to the genetic background of each strain. This possibility adds to the value of repeating the transcriptional studies for mutant and parent bacteria. Alternatively, it is possible the mechanism responsible for enhanced fitness is the same in all three backgrounds examined. We observe a gradient in the promotion of in vivo fitness, with AK1 displaying the least advantage over parent followed by JD1 and JD4. This gradient is also reflected in many of the shared transcriptional changes captured in the RNA sequencing data. It is possible that decreases in sensitivity to antimicrobial peptides and oxidative stress in JD1 and AK1 are subtle enough to miss using in vitro measures of assessment, but substantial enough to provide a competitive edge over parent bacteria when the competition continues over days. Because of this possibility, conducting parallel competitive infections between JD1, AK1 and the respective parent strains in wild-type BALB/c and CRAMP deficient BALB/c mice may provide value.

Why a fluoroquinolone resistance-conferring gyrA mutant is able to enhance in vivo fitness across multiple strains of $N$. gonorrhoeae is an interesting question. Having evolved as a well-adapted human-specific pathogen, $N$. gonorrhoeae has acquired genomic determinants that are best suited for in vivo fitness. If the $g y r A_{91 / 95}$ allele provides the gonococcus with a survival advantage over the defined wild-type gyrA, nature should have selected the seemingly more fit gyrA91/95 during this organism's evolution. It is possible that the in vivo advantage we see in our experimental murine model of infection is limited to state of an established infection. The gyrA $A_{91 / 95}$ mutants may have a transmission disadvantage relative to the wild-type gyrA gonococcus, which would explain the apparent lack of selection for the gyrA $_{91 / 95}$ allele. 
Another possibility is that the gyrA allele that we call wild-type is actually a compensatory mutation selected for as a compromise at some point after the antibiotic era ensued. As mentioned previously, antibiotic therapy for gonococcal disease began with the administration of sulfa drugs in the 1930's. In the decades that followed, the gonococcus was challenged with several classes of antibiotics and multiple generations within some of these classes. It is possible $g_{y r} A_{91 / 95}$ is the true wild-type allele, and the gonococcus acquired the gyrA allele we currently refer to as "wild-type" at some point following decades of antibiotic exposure. Adaptation to treatment in the human host through the acquisition of antibiotic resistance determinants over time may have influenced the selection of a new gyrA allele best suited for evolving fitness requirements during the era of antibiotic pressure. With this hypothesis, we would expect $g_{y} A_{91 / 95}$ to be the dominant allele encountered in the gonococcus during the pre-antibiotic era, and remaining $g_{y r} A_{91 / 95}$ alleles in the post-antibiotic era would contain fewer resistance determinants to additional classes of antibiotics. One interesting study consistent with this idea involved a 1998 assessment of intercity gonoccocal isolates within the United States displaying decreased susceptibility to fluoroquinolones. With a total of 783 clinical isolates from gonorrhea patients in five cities, Kilmarx and colleagues found that all of the isolates displaying decreased susceptibility to ciprofloxacin were $\beta$-lactamase negative and susceptible to spectinomycin, ceftriaxone, and cefixime (44). It is possible that these isolates did not contain an evolved gyrA providing decreased susceptibility to fluoroquinolones, but rather an allele was not as evolved as other gonococcal isolates containing a greater number of resistance determinants. It would be interesting to investigate the possibility that gyrA $_{91 / 95}$ is less fit than what we currently refer to as "wild- 
type” gryA if these isolates contained some combination of other classes of resistance determinants.

In summary, these experiments suggest that in one strain of $N$. gonorrhoeae, an increased degree of tolerance to host defenses is driven by a resistance-conferring mutant GyrA allele, which may help explain the rapid proliferation of FQ-resistant $N$.

gonorrhoeae; Future efforts should help elucidate the mechanism by which mutant JD4 is more resistant to host-produced CAMPs, which may be novel, as well as identify the basis of gyrA $_{91 / 95}$-driven in other gonococcal strains. 


\section{Appendix: The Impact of Fluoroquinolone Resistance-conferring gyrA and parC Mutations on Fitness and the Role of Compensatory Mutations}

\section{Background}

As mentioned previously, antibiotic resistance provides a selective advantage for bacteria grown in the presence of antibiotic pressure, yet bacteria that contain antibiotic resistance mechanisms often carry the burden of reduced fitness relative to antibiotic susceptible parent strains $(3 ; 7 ; 17 ; 58)$. Many of the fitness costs associated with antibiotic resistance are accompanied by additional energy requirements needed for overexpression of genes involved in resistance, or the maintenance of extra-chromosomal elements responsible for conferring resistance. Compensatory mutations, either within the genes that confer resistance or in genes that are unrelated to resistance, can provide bacteria with adaptive means of overcoming fitness constraints associated with resistance to antibiotics $(4 ; 46 ; 47 ; 58 ; 70)$.

In this appendix, I describe the work I have done to investigate the occurrence of compensatory mutations that restore fitness to gonococci possessing multiple antibiotic resistance mutations, specifically gyr $A_{91 / 95}$ and $\operatorname{par}_{86}$ mutations that confer high level ciprofloxacin resistance and $m t r$ locus mutations that confer resistance to macrolide antibiotics and high level penicillin.

In our work with gyrA and parC mutations, we found that a strain with an $m t r R$ promoter mutation ( $\left.m t_{r} R_{-79}\right)$ and the first and second-step type 2 topoisomerase mutations in gyrA and parC exhibited the greatest fitness cost with respect to growth in vitro and 
recovery from experimentally infected mice. This mutant, AK12, was derived from mutant AK11 (gyrA $\left.{ }_{91 / 95}, m t r R_{-79}\right)$, and interestingly, a compensatory mutant of strain AK12 was selected during an in vivo competition experiment between mutants AK12 and AK11. This mutant, called AK13, was isolated on day 5 post-inoculation from a mouse that initially showed a significant drop in the total number of CFU recovered and in the recovery of AK12 relative to AK11, followed by a return of high numbers of gonococci, all of which were highly ciprofloxacin resistant. Mutant AK13 also showed a pronounced growth advantage compared to its parent AK12 and the wild type strain. Since MIC analysis of AK13 showed no difference in the resistance to ciprofloxacin compared to AK12, we hypothesized that the means of over-coming the fitness burden displayed by AK12 was not through repair of quinolone-conferring mutations, but rather through the acquisition of one or more compensatory mutations.

The parent of strains AK11, AK12, and AK13 is KH15, which is the parent strain FA19 containing an $m t r R_{-79}$ mutation. This mutation is a single base deletion in the inverted repeat sequence between the -10 and -35 regions of the $m t r R$ promoter (36). This single base deletion in KH15, AK11, and AK12 disrupts the optimal spacing between the -10 and -35 elements required for RNA polymerase association and subsequent transcription. Therefore, $m$ trR is not efficiently transcribed in KH15, AK11, and AK12 allowing for over-expression of the mtrCDE efflux pump. Since our laboratory previously defined a fitness gradient for $m t r C D E$ expression for different types of $m t r$ locus mutations $(97 ; 98)$, we hypothesized that the $m t r R$ promoter region or structural gene in AK13 may contain additional mutations that help restore fitness. We also speculated that changes may be present in the QRDR region of AK13. Therefore, 
we sequenced the intergenic region between mtrR and $m \operatorname{tr} C D E$ as well as the full-length parC and gyrA genes in strains FA19, AK12, and AK13 in an attempt to identify potential compensatory mutations that would help explain AK13's in vivo fitness enhancement. Sequence analysis of the parC gene showed no differences between mutants AK13 and AK12, and the presence of a D86N mutation compared to strain FA19 as expected. Similarly, the gyrA gene of AK12 contains a S91F and D95N amino acid changes relative to strain FA19 as expected. However, as shown in Fig. 12, AK13 carries a point mutation in the third nucleotide of codon 91 of the gyrA gene. Relative to parent AK12, this TTC $\rightarrow$ TTA mutation leads to a F91L substitution in the QRDR of gyrA. When we looked at the mtrR upstream sequence from AK13, we also saw an insertion mutation within the inverted repeat sequence of the promoter that restores the spacing of the -10 and -35 sequences needed for $m t r R$ expression and natural repression of the $m \operatorname{trCDE}$ operon. How the $m t r R$ promoter insertion mutation or the F91L mutation in gyrA may lead to elevated bacterial fitness is not known. It is very possible that additional fitness-enhancing mutations exist in AK13, and whole genome sequencing is needed to explore this possibility.

While the mechanism by which the fitness of $\mathrm{Cip}^{\mathrm{R}}$ mutant $\mathrm{AK} 13$ was restored is not yet known, we conclude that selection for compensatory mutations is a mechanism by which fitness can be restored in highly $\mathrm{Cip}^{\mathrm{R}}$ strains. These data were published in the manuscript entitled "Impact of fluoroquinolone resistance mutations on gonococcal fitness and in vivo selection for compensatory mutations” (47). To continue to investigate the hypothesis that compensatory mutations may allow high $\mathrm{Cip}^{\mathrm{R}}$ gonococci to be maintained in the absence of fluoroquinolone treatment, we introduced the second 
step parC $C_{86}$ mutation in gyrA $_{91 / 95}$ mutants of strains FA1090 and MS11 (mutants JD1 and JD4, respectively). We predicted that the resultant mutants, JD1.2 and JD4.2 would exhibit elevated ciprofloxacin resistance and an associated fitness cost. We also hypothesized that procurement of compensatory mutations in the fitness-burdened $\mathrm{Cip}^{\mathrm{R}}$ mutants of $N$. gonorrhoeae will elevate in vivo fitness without sacrificing the magnitude of antibiotic resistance 
Figure 12. Highlighted QRDR GyrA and parC amino acid sequences of $\operatorname{Cip}^{\mathrm{S}}$ and $\mathrm{Cip}^{\mathrm{R}}$ Neisseria gonorrhoeae

$\mathrm{Cip}^{\mathrm{S}}$ Neisseria gonorrhoeae (FA19), $\mathrm{Cip}^{\mathrm{R}}$ (AK12), and the $\mathrm{Cip}^{\mathrm{R}}$ compensatory mutant (AK13) are shown at the top of the figure. Shown at the bottom is the intergenic promoter region of the $m t r C D E$ efflux pump repressor ( $m t r \mathrm{R})$. 
gyrA (QRDR)

91

95

tac cac cce cac gge gat tce gca gtt tac gac acc atc gtc ctg atg gcg

$\begin{array}{lllllllllllllllllllllllll}\text { FA19 } & \text { Y } & \text { H } & \text { P } & \text { H } & \text { G } & \text { D } & \text { S } & \text { A } & \text { V } & \text { Y } & \text { D } & \text { T } & \text { I } & \text { V } & R & \text { M } & \text { A }\end{array}$

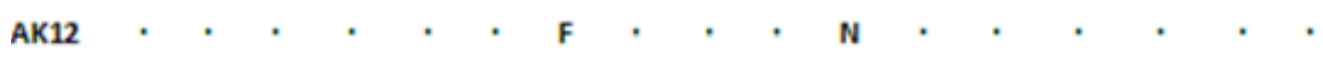

АK13

$\operatorname{parC}(\mathrm{QRDR})$

86

ggt aaa tac cat cog cac ggc gac agt tce gcc tat gag gcg atg gtg cgc

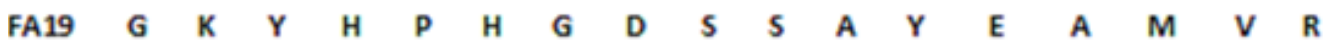

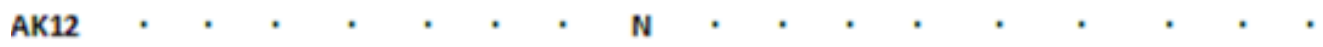

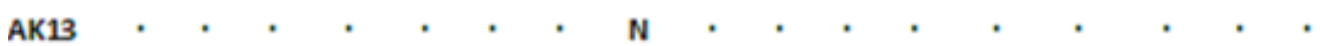

$\underline{m \text { trR-mtrCDE Intergenic Region }}$

FA19 g g g c g gatt a t a a a a a ga ctt t t t a t c c g t g c a a t c g t

AK12 . . . . . . . . . . . . . . . . . . . . . . . . . .

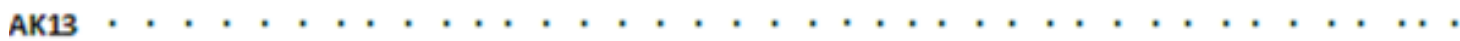




\section{Hypothesis 2:}

Procurement of compensatory mutations in the fitness-burdened $\mathrm{Cip}^{\mathrm{R}}$ mutants of $N$. gonorrhoeae will elevate in vivo fitness without sacrificing the magnitude of antibiotic resistance.

\section{Materials and methods}

Bacterial strains and mutant construction

Neisseria gonorrhoeae gyrA91/95 mutants JD1 and JD4 were used as parents for generation of $\mathrm{Cip}^{\mathrm{R}}$ bacteria containing the $\operatorname{par}_{86}$ allele that provides this elevated resistance. JD1.2 and JD4.2 gyr $A_{91 / 95}$ and $\operatorname{par}_{86}$ mutants were constructed by allelic exchange with donor PCR amplicon containing the Asp86Asn mutation. The method employed followed that previously reported for the construction of AK2 and AK12 (35; 47). Briefly, a 1.6-kb region of parC was amplified from AK2 using parC1-for and parC1-rev primers from Table 2. JD1 and JD4 were passed on non-selective solid media following an initial sub culture. Bacteria were suspended in PBS and adjusted to an $\mathrm{A}_{600}$ of 0.05. Ten microliters of the bacterial suspension were spotted onto solid non-selective GC media followed by direct overlay of approximately $1 \mu \mathrm{g}$ of template DNA used for targeted homologous recombination. Following incubation at $37^{\circ} \mathrm{C}$ for 24 hours, individual colonies were isolated and streaked onto solid growth media containing $2 \mu \mathrm{g} / \mathrm{ml}$ of ciprofloxacin for selection of gyrA $_{91 / 95} /$ parC $_{86}$ mutants. Freezer stocks were 
generated, and mutant bacteria were sequenced for positive identification of the altered nucleotide sequence (Figures 13 and 14). 
Figure 13. Open reading frame sequence of the parC sequence from JD1.2.

The highlighted codon shows the GAC $\rightarrow$ AAC point mutation that produces the Asp $\rightarrow$ Asn amino acid transition at position 86 (D86N). 
ATGAATACGCAACCGCACGCTTCCCATACCGATTCCAACACGCTGATGCTCGGCCGATACGCCGAACGCGC CTATCTCGAATACGCCATGAGCGTGGTCAAAGGCCGCGCGCTGCCTGAAGTTTCAGACGGCCAAAAGCCCG TGCAGCGGCGCATTTTGTTTGCCATGCGCGATATGGGTTTGACGGCGGGGGCGAAGCCGGTGAAATCGGCG CGCGTGGTCGGCGAGATTTTGGGTAAATACCATCCGCACGGCAACAGTTCCGCCTATGAGGCGATGGTGCG CATGGCTCAGGATTTTACCTTGCGCTATCCCTTAATCGACGGCATCGGCAACTTCGGTTCGCGCGACGGCG ACGGGGCGGCGGCGATGCGTTACACCGAAGCGCGGCTCACGCCGATTGCGGAATTGCTGTTGTCCGAAATC AATCAGGGGACGGTGGATTTTATGCCGAACTACGACGGCGCGTTTGACGAGCCGCTGCACCTTCCCGCCCG CTTGCCTATGGTGTTGCTCAACGGCGCGTCGGGCATCGCGGTGGGTATGGCGACCGAGATTCCGTCGCACA ATTTGAACGAAGTCACGCAGGCGGCGATTGCACTGTTGAAGAAACCGACGCTGGAAACCGCCGACCTGATG CAATATATTCCTGCTCCCGATTTTGCCGGCGGCGGTCAAATCATCACGCCGGCGGACGAATTGCGCCGTAT TTACGAAACCGGCAAGGGCAGCGTGCGCGTGCGTGCGCGTTATGAAATCGAGAAATTGGCGCGCGGACAGT GGCGCGTCATCGTAACCGAACTGCCGCCGAACGCCAATTCCGCCAAAATCCTTGCCGAAATCGAAGAGCAA ACCAACCCGAAACCGAAAGCGGGCAAGAAGCAGCTCAACCAAGACCGGCTTAATACCAAAAAGCTGATGCT GGATTTAATCGACCGCGTGCGCGACGAGTCCGACGGCGAACATCCCGTGCGCCTTGTATTTGAACCGAAAT CCAGCCGCATCGATACCGATACCTTCATCAACACGCTGATGGCGCAAACTTCGCTGGAAGGCAATGTGTCC ATGAACTTGGTGATGATGGGTTTGGACAACCGCCCCGCGCAGAAAAACCTGAAAACGATTTTTGCAGGAATG GCTGGATTTCCGCATCGTTACCGTAACACGCCGTCTGAAATTCCGTTTAAACCAAGTGGAAAAACGGCTGC ACATCCTCGAAGGCCGTCTGAAAGTCTTTCTGCACATCGACGAAGTGATTAAAGTCATCCGCGAATCGGAC GACCCGAAAGCCGATTTGATGGCGGTGTTCGGGCTGACCGAAATCCAGGCCGAAGACATTTTTGAAATCCG CCTGCGTCAGTTGGCGCGTTTGGAAGGTTTCAAACTCGAAAAAGAATTGAACGAATTGCGCGAAGAACAAG GCCGTCTGAATATCTTTTTGGGCGACGAAAACGAAAAACGCAAGCTGATTATCAAAGAGATGCAGGCGGAC ATGAAGCAGTTCGGCGACGCGCGCCGCACGCTGGTGGAAGAGGCCGGACGCGCCGTGCTGACACAAACCGC CGCCGACGAACCCATCACGCTGATTTTGTCGGAAAAAGGCTGGATACGCAGCCGTGCCGGACATAATCTCG ATTTGAGCCAAACCGCGTTCAAAGAAGGCGACCGCCTCAAACAAACCCTTGAAGGCCGCACTGTTTTACCC GTCGTCATCCTCGATTCATCGGGCAGAACCTACTCGATCGATGCCGCCGAAATCCCCGGCGGACGCGGCGA CGGCGTACCGGTTTCCTCCTTAATCGAGTTGCAAAACGGCGCGAAACCCGTCGCGATGTTGACAGGATTGC CGGAACAACATTATTTATTATCAAGCAGCGGCGGCTACGGCTTTATCGCCAAGCTGGGCGATATGGTCGGA CGCGTGAAAGCGGGCAAAGTGGTGATGACCGCAGACAGCGGCGAAACCGTCCTGCCGCCGGTTGCCGTCTA TGCCTCCTCGTTCATCAACCCCGACTGCAAAATCATTGCAGCCACCAGTCAAAACCGCGCCCTCGCCTTCC CCATCGGCGAATTGAAAATTATGGCGAAAGGCAAAGGACTGCAAATCATCGGATTAAACGCCGGCGAATCG ATGACGCATACCGCCGTTTCTTCCGAGCCGGAAATCCTGATTGAAAGCGAAGGCAGGCGCGGCGCGGCGCA CAAAGACCGCCTCCCCGTCGCCCTGATTGAGGCAAAACGCGGCAAAAAAGGCAGACTGTTGCCCATATCGG GCAGCCTGAAACAGCTTTCTTCCCCCAAATAA 
Figure 14. Open reading frame sequence of the parC sequence from JD4.2.

The highlighted codon shows the GAC $\rightarrow$ AAC point mutation that produces the Asp $\rightarrow$ Asn amino acid transition at position 86 (D86N). 
ATGAATACGCAACCGCACGCTTCCCATACCGATTCCAACACGCTGATGCTCGGCCGATACGCCGAACGCGC CTATCTCGAATACGCCATGAGCGTGGTCAAAGGCCGCGCGCTGCCTGAAGTTTCAGACGGCCAAAAGCCCG TGCAGCGGCGCATTTTGTTTGCCATGCGCGATATGGGTTTGACGGCGGGGGCGAAGCCGGTGAAATCGGCG CGCGTGGTCGGCGAGATTTTGGGTAAATACCATCCGCACGGCAACAGTTCCGCCTATGAGGCGATGGTGCG CATGGCTCAGGATTTTACCTTGCGCTATCCCTTAATCGACGGCATCGGCAACTTCGGTTCGCGCGACGGCG ACGGGGCGGCGGCGATGCGTTACACCGAAGCGCGGCTCACGCCGATTGCGGAATTGCTGTTGTCCGAAATC AATCAGGGGACGGTGGATTTTATGCCGAACTACGACGGCGCGTTTGACGAGCCGCTGCACCTTCCCGCCCG CTTGCCTATGGTGTTGCTCAACGGCGCGTCGGGCATCGCGGTGGGTATGGCGACCGAGATTCCGTCGCACA ATTTGAACGAAGTCACGCAGGCGGCGATTGCACTGTTGAAGAAACCGACGCTGGAAACCGCCGACCTGATG CAATATATTCCTGCTCCCGATTTTGCCGGCGGCGGTCAAATCATCACGCCGGCGGACGAATTGCGCCGTAT TTACGAAACCGGCAAGGGCAGCGTGCGCGTGCGTGCGCGTTATGAAATCGAGAAATTGGCGCGCGGACAGT GGCGCGTCATCGTAACCGAACTGCCGCCGAACGCCAATTCCGCCAAAATCCTTGCCGAAATCGAAGAGCAA ACCAACCCGAAACCGAAAGCGGGCAAGAAGCAGCTCAACCAAGACCGGCTTAATACCAAAAAGCTGATGCT GGATTTAATCGACCGCGTGCGCGACGAGTCCGACGGCGAACATCCCGTGCGCCTTGTATTTGAACCGAAAT CCAGCCGCATCGATACCGATACCTTCATCAACACGCTGATGGCGCAAACTTCGCTGGAAGGCAATGTGTCC ATGAACTTGGTGATGATGGGTTTGGACAACCGCCCCGCGCAGAAAAACCTGAAAACGATTTTTGCAGGAATG GCTGGATTTCCGCATCGTTACCGTAACACGCCGTCTGAAATTCCGTTTAAACCAAGTGGAAAAACGGCTGC ACATCCTCGAAGGCCGTCTGAAAGTCTTTCTGCACATCGACGAAGTGATTAAAGTCATCCGCGAATCGGAC GACCCGAAAGCCGATTTGATGGCGGTGTTCGGGCTGACCGAAATCCAGGCCGAAGACATTTTTGAAATCCG CCTGCGTCAGTTGGCGCGTTTGGAAGGTTTCAAACTCGAAAAAGAATTGAACGAATTGCGCGAAGAACAAG GCCGTCTGAATATCTTTTTGGGCGACGAAAACGAAAAACGCAAGCTGATTATCAAAGAGATGCAGGCGGAC ATGAAGCAGTTCGGCGACGCGCGCCGCACGCTGGTGGAAGAGGCCGGACGCGCCGTGCTGACACAAACCGC CGCCGACGAACCCATCACGCTGATTTTGTCGGAAAAAGGCTGGATACGCAGCCGTGCCGGACATAATCTCG ATTTGAGCCAAACCGCGTTCAAAGAAGGCGACCGCCTCAAACAAACCCTTGAAGGCCGCACTGTTTTACCC GTCGTCATCCTCGATTCATCGGGCAGAACCTACTCGATCGATGCCGCCGAAATCCCCGGCGGACGCGGCGA CGGCGTACCGGTTTCCTCCTTAATCGAGTTGCAAAACGGCGCGAAACCCGTCGCGATGTTGACAGGATTGC CGGAACAACATTATTTATTATCAAGCAGCGGCGGCTACGGCTTTATCGCCAAGCTGGGCGATATGGTCGGA CGCGTGAAAGCGGGCAAAGTGGTGATGACCGCAGACAGCGGCGAAACCGTCCTGCCGCCGGTTGCCGTCTA TGCCTCCTCGTTCATCAACCCCGACTGCAAAATCATTGCAGCCACCAGTCAAAACCGCGCCCTCGCCTTCC CCATCGGCGAATTGAAAATTATGGCGAAAGGCAAAGGACTGCAAATCATCGGATTAAACGCCGGCGAATCG ATGACGCATACCGCCGTTTCTTCCGAGCCGGAAATCCTGATTGAAAGCGAAGGCAGGCGCGGCGCGGCGCA CAAAGACCGCCTCCCCGTCGCCCTGATTGAGGCAAAACGCGGCAAAAAAGGCAGACTGTTGCCCATATCGG GCAGCCTGAAACAGCTTTCTTCCCCCAAATAA

In vitro growth measurements 
Mutant and parent strain bacteria were grown on solid media for 18 hours, and suspensions were made in GCB. Each bacterial suspension was passed through a 1.2 micron filter to discourage aggregate formation, and the $\mathrm{A}_{600}$ was adjusted to 0.075 into $30 \mathrm{ml}$ of GCB. Thirty milliliter cultures for each mutant and parent strain set were incubated under agitation at $37^{\circ} \mathrm{C}$. Optical density recordings were taken each hour, and the average time needed to reach an $\mathrm{A}_{600}$ of 1.0 was established. All growth curves were run in duplicate.

For assessing relative in vitro fitness, competition experiments were carried out using similar numbers of the mutant and parent strain being compared. Each mixed culture was initiated at an $\mathrm{A}_{600}$ of approximately 0.08 and $100 \mu \mathrm{l}$ aliquots were plated onto selective (0.125 $\mu \mathrm{g} / \mathrm{ml}$ ciprofloxacin) and non-selective solid media. Mutant to parent ratios were obtained by subtracting the CFUs on the selective plates from the CFUs on the non-selective plates. Subsequent $100 \mu$ l aliquots were collected and plated in the same manner every 2 hours until an $\mathrm{A}_{600}$ of 1.0 was achieved. A competitive index (CI) was established by dividing the ratio of mutant to parent CFUs recorded from the plates at each time point by the ratio of input mutant to parent CFUs. CI values greater than 1 illustrates enhanced fitness of the mutant relative to parent; whereas, CI values less than 1 illustrate depressed mutant fitness relative to parent.

\section{MIC measurements}


Minimum inhibitory concentrations (MICs) of ciprofloxacin were established for mutant and parent strain bacteria using a standard agar dilution assay (23). Reported ranges of intermediate level resistance and high level resistance to ciprofloxacin were reported in accordance with CDC guidelines.

In vivo competitive infections

BALB/c mice (approximately 8 weeks old) in the diestrus stage of the estrous cycle were administered 17- $\beta$-estradiol subcutaneously to optimize susceptibility to GC infection $2.4 \mathrm{mg}$ streptomycin and $0.4 \mathrm{mg}$ of vancomycin were injected intraperitoneally twice each day and $0.04 \mathrm{~g}$ of trimethoprim sulfate per $100 \mathrm{ml}$ of drinking water was provided throughout the course of the experiment to minimize growth of commensal flora. Mutant and parent strain N. gonorrhoeae were grown on solid media for 18 hours, and suspensions were passed through a 1.2 micron filter to minimize aggregations. Mutant and parent strains were adjusted to equal $A_{600}$ optical densities and mixed in a 1 to 1 ratio. Mixed $20 \mu \mathrm{l}$ inocula were introduced intravaginally into mice, and each inoculum was plated on both non-selective and selective $(0.125 \mu \mathrm{g} / \mathrm{ml}$ ciprofloxacin) solid media to establish input bacteria ratios. Vaginal swab cultures performed on days 1, 3,5 , and 7 served as output bacteria ratios. A competitive index was calculated by dividing the ratio of mutant to parent CFUs from each culture day by the ratio of input mutant to parent CFUs. CI values greater than 1 illustrates enhanced fitness of the mutant relative to parent; whereas, CI values less than 1 illustrate depressed mutant fitness relative to parent. 


\section{Results}

To investigate the phenomenon of selection for compensatory mutations as a mechanism for restoring fitness in the context of ciprofloxacin resistant mutants, we first aimed to move the second-step $\operatorname{Cip}^{\mathrm{R}}$ mutation in $\operatorname{parC}$ to strains JD1 and JD4. We named these strains JD1.2 and JD4.2 respectively. After establishing that JD1.2 and JD4.2 had the predicted the Cip $^{\mathrm{R}}$ phenotype (Table 1), we assessed in vitro growth by culturing each strain separately in liquid culture or together with its ciprofloxacin susceptible parent strain. While there appeared to be no difference between JD1.2 and FA1090 when cultured independently, surprisingly, mutant JD4.2 grew faster than wildtype MS11. JD4.2 also displayed greater in vitro fitness than MS11 during co-culture measurements (Fig. 15).

We next conducted competitive infections in the murine model to assess the relative in vivo fitness of JD1.2 to FA1090 and JD4.2 to MS11 (Fig. 16). Fitness studies with mutant JD1.2 supported our hypothesis that the $\mathrm{Cip}^{\mathrm{R}}$-conferring mutation in parC would reduce fitness relative to parental strains of $N$. gonorrhoeae. Cultures taken on

days 3 and 5, all showed decreased fitness for JD1.2. However, in one mouse only Cip ${ }^{\mathrm{R}}$ bacteria were recovered on Day 7 post inoculation, suggesting selection for a compensatory mutation had occurred during infection. This strain was isolated and frozen for further investigation.

In contrast to mutant JD1.2, mutant JD4.2 displayed a high competitive index relative to MS11 throughout the entire infection period. Based on the increased growth of JD1.2 in vitro relative to MS11 and the enhanced fitness of JD1.2 in vivo, we speculate that during the process of mutating the parC allele of JD4, a spontaneous mutation arose 
elsewhere in the genome which resulted in elevated fitness. While this remains an interesting finding and should be pursued, the parC mutation should be reintroduced into JD4 for future studies on in-host selection for compensatory mutations in Cip ${ }^{\mathrm{R}}$ MS11 mutant bacteria carrying gyrA, parC mutations. 
Figure 15. Growth curves for MS11, FA1090, and the two Cip ${ }^{\mathrm{R}}$ mutants

The two Cip ${ }^{\mathrm{R}}$ mutants JD1.2 and JD4.2 are shown in panel A. Panels B and C show results of the in vitro co-cultures between JD4.2 vs. MS11 and JD1.2 vs. FA1090, respectively. 
A

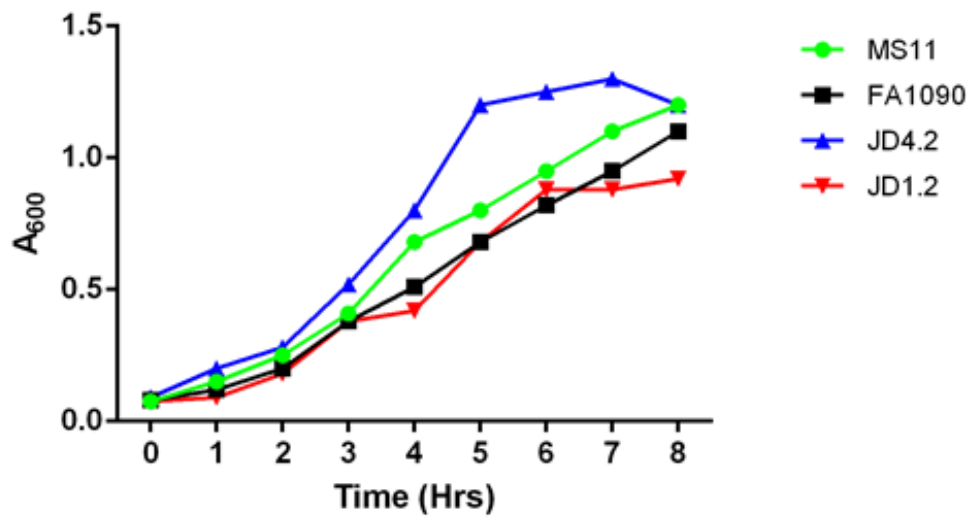

B

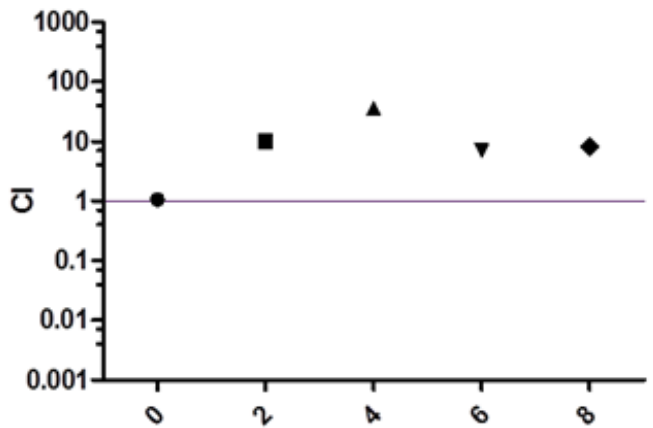

Time (Hrs)

C

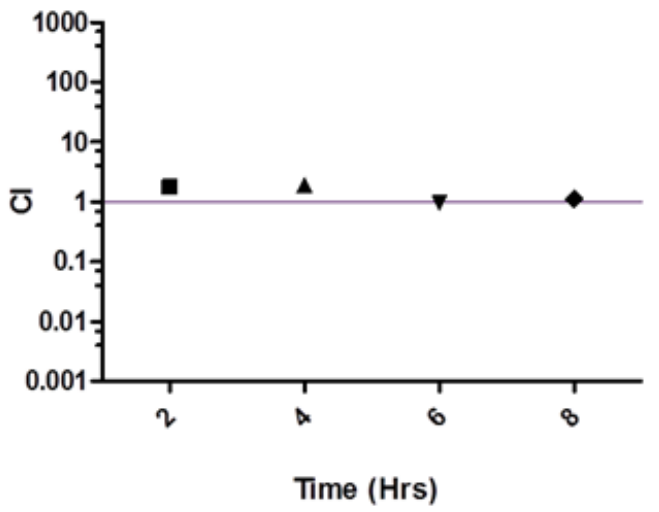


Figure 16. In vivo assessment of fitness between parental bacteria and the $g y r A_{91 / 95}$ and $\operatorname{parC}_{86} \operatorname{Cip}^{\mathrm{R}}$ mutants

The gyrA $_{91 / 95}$ and $\operatorname{par}_{86}$ Cip $^{\mathrm{R}}$ mutants in the FA1090 and FA19 backgrounds display an in vivo fitness burden in competition experiments with isogenic parents using the mouse model of infection. However, Cip $^{\mathrm{R}}$ mutant in an MS11 background exhibits a fitness advantage over parent bacteria. The geometric means of repeat experiments are presented. Black symbols represent the first experiment, and blue symbols represent the second. Open symbols are shown for vaginal cultures yielding only mutant bacteria. 

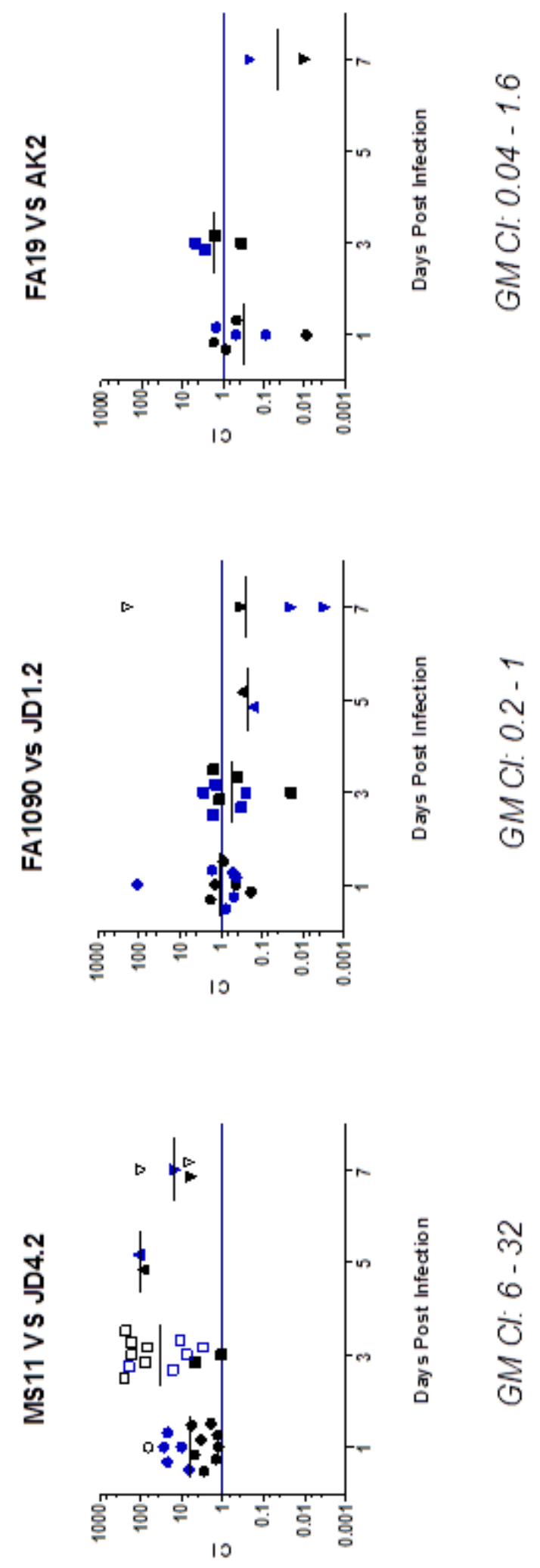


\section{Discussion and Future Directions}

The in vivo fitness burden of high level resistance to ciprofloxacin in Neisseria gonorrhoeae can be overcome through the acquisition of compensatory mutations, and understanding the nature of these mutations will provide a better understanding of specific bacterial mechanisms that can directly contribute to the fitness of Neisseria gonorrhoeae. Some of these fitness benefits may act at the level of increased growth and be observed both in vitro and in vivo, such as was seen for AK13. Others may only occur in vivo. The in vitro growth of compensatory mutant JD1.4 was not examined and thus at this time, we do not know if the observed fitness restoration occurs only in the context of the host.

Continued investigation of the mutations found in AK13 is needed to understand the basis of the compensatory phenotype. The F91L amino acid and the repaired $m t r R$ promoter may be the sole source of the enhanced fitness observed with AK13; however, why repair of the $m t r R$ mutation, which is predicted to reduce fitness, contributes to the in vivo phenotype of AK13 is not known and it is possible that none of these mutations contributes to enhanced fitness. To address this issue, the GyrA F91L allele could be transferred to the parent strain AK12, followed by repeat in vitro and in vivo measures of growth. If no evidence is obtained in support of the GyrA F91L being responsible for the compensatory phenotype shown by AK13, whole genome sequencing should be carried out for a more comprehensive analysis. Similarly, growth kinetics and repeat testing of in vivo fitness should be conducted to analyze the putative compensatory mutant isolated from the JD1.2, and whole genome sequencing performed if studies confirm the compensatory phenotype. These experiments may reveal new information about 
gonococcal adaptation mechanisms as well as identify compensatory mutations that may also be present in $\mathrm{Cip}^{\mathrm{R}}$ clinical isolates. 


\section{REFERENCES}

1. http://www.cdc.gov/std/gonorrhea/default.htm.

2. 1995. Global prevalence and incidence of selected curable sexually transmitted diseases : overview and estimates. Geneva: World Health Organization. 26 p. + annex. pp.

3. Abdelraouf K, Kabbara S, Ledesma KR, Poole K, Tam VH. 2011. Effect of multidrug resistance-conferring mutations on the fitness and virulence of Pseudomonas aeruginosa. The Journal of Antimicrobial Chemotherapy 66:1311-7

4. Albarracin Orio AG, Pinas GE, Cortes PR, Cian MB, Echenique J. 2011. Compensatory evolution of $p b p$ mutations restores the fitness cost imposed by beta-lactam resistance in Streptococcus pneumoniae. PLoS pathogens 7:e1002000

5. $\quad$ Aldred KJ, McPherson SA, Turnbough CL, Jr., Kerns RJ, Osheroff N. 2013. Topoisomerase IV-quinolone interactions are mediated through a water-metal ion bridge: mechanistic basis of quinolone resistance. Nucleic Acids Research 41:4628-39

6. Andersson DI. 2006. The biological cost of mutational antibiotic resistance: any practical conclusions? Curr Opin Microbiol 9:461-5

7. Andersson DI, Levin BR. 1999. The biological cost of antibiotic resistance. Curr Opin Microbiol 2:489-93

8. Arko RJ. 1972. Neisseria gonorrhoeae: experimental infection of laboratory animals. Science 177:1200-1

9. Arko RJ. 1989. Animal models for pathogenic Neisseria species. Clinical Microbiology Reviews 2 Suppl:S56-9

10. Arko RJ, Kraus SJ, Brown WJ, Buchanan TM, Kuhn US. 1974. Neisseria gonorrhoeae: effects of systemic immunization on resistance of chimpanzees to urethral infection. The Journal of Infectious Diseases 130:160-4

11. Arko RJ, Wong KH. 1977. Comparative physical and immunological aspects of the chimpanzee and guinea-pig subcutaneous chamber models of Neisseria gonorrhoeae infection. The British Journal of Venereal Diseases 53:101-5 
12. Bagel S, Hullen V, Wiedemann B, Heisig P. 1999. Impact of gyrA and parC mutations on quinolone resistance, doubling time, and supercoiling degree of Escherichia coli. Antimicrobial Agents and Chemotherapy 43:868-75

13. Balsalobre L, Ferrandiz MJ, de Alba G, de la Campa AG. 2011. Nonoptimal DNA topoisomerases allow maintenance of supercoiling levels and improve fitness of Streptococcus pneumoniae. Antimicrobial Agents and Chemotherapy 55:1097105

14. Barlow D, Phillips I. 1978. Gonorrhoea in women. Diagnostic, clinical, and laboratory aspects. Lancet 1:761-4

15. Bates AD, Berger JM, Maxwell A. 2011. The ancestral role of ATP hydrolysis in type II topoisomerases: prevention of DNA double-strand breaks. Nucleic Acids Research 39:6327-39

16. Bielecki P, Muthukumarasamy U, Eckweiler D, Bielecka A, Pohl S, et al. 2014. In vivo mRNA profiling of uropathogenic Escherichia coli from diverse phylogroups reveals common and group-specific gene expression profiles. mBio 5:e01075-14

17. Bjorkman J, Andersson DI. 2000. The cost of antibiotic resistance from a bacterial perspective. Drug resistance updates : Reviews and Commentaries in Antimicrobial and Anticancer Chemotherapy 3:237-45

18. Blake MS, Wetzler LM. 1995. Vaccines for gonorrhea: where are we on the curve? Trends in Microbiology 3:469-74

19. Bos MP, Hogan D, Belland RJ. 1997. Selection of Opa+ Neisseria gonorrhoeae by limited availability of normal human serum. Infection and Immunity 65:645-50

20. Boslego JW, Tramont EC, Chung RC, McChesney DG, Ciak J, et al. 1991. Efficacy trial of a parenteral gonococcal pilus vaccine in men. Vaccine 9:154-62

21. Brown WJ, Lucas CT, Kuhn US. 1972. Gonorrhoea in the chimpanzee. Infection with laboratory-passed gonococci and by natural transmission. The British Journal of Venereal Diseases 48:177-8

22. Burch CL, Danaher RJ, Stein DC. 1997. Antigenic variation in Neisseria gonorrhoeae: production of multiple lipooligosaccharides. Journal of Bacteriology 179:982-6

23. Centers for Disease C, Prevention. 2014. Recommendations for the laboratorybased detection of Chlamydia trachomatis and Neisseria gonorrhoeae--2014. MMWR. Recommendations and reports : Morbidity and mortality weekly report. Recommendations and reports / Centers for Disease Control 63:1-19 
24. Cohen MS, Cannon JG. 1999. Human experimentation with Neisseria gonorrhoeae: progress and goals. The Journal of Infectious Diseases 179 Suppl 2:S375-9

25. Cousin SL, Jr., Whittington WL, Roberts MC. 2003. Acquired macrolide resistance genes and the 1 bp deletion in the $m$ trR promoter in Neisseria gonorrhoeae. The Journal of Antimicrobial Chemotherapy 51:131-3

26. Edwards JL, Apicella MA. 2004. The molecular mechanisms used by Neisseria gonorrhoeae to initiate infection differ between men and women. Clinical Microbiology Reviews 17:965-81, table of contents

27. Emmerson AM, Jones AM. 2003. The quinolones: decades of development and use. The Journal of Antimicrobial Chemotherapy 51 Suppl 1:13-20

28. Evans BA. 1976. Detection of gonorrhoea in women. The British Journal of Venereal Diseases 52:40-2

29. Everard A, Lazarevic V, Gaia N, Johansson M, Stahlman M, et al. 2014. Microbiome of prebiotic-treated mice reveals novel targets involved in host response during obesity. The ISME Journal 8:2116-30

30. Forest KT, Tainer JA. 1997. Type-4 pilus-structure: outside to inside and top to bottom--a minireview. Gene 192:165-9

31. Furuse Y, Finethy R, Saka HA, Xet-Mull AM, Sisk DM, et al. 2014. Search for microRNAs expressed by intracellular bacterial pathogens in infected mammalian cells. PLoS One 9:e106434

32. Gradison M. 2012. Pelvic inflammatory disease. American Family Physician 85:791-6

33. Greenberg L. 1975. Field trials of a gonococcal vaccine. The Journal of Reproductive Medicine 14:34-6

34. Greenberg L, Diena BB, Ashton FA, Wallace R, Kenny CP, et al. 1974. Gonococcal vaccine studies in Inuvik. Canadian Journal of Public Health = Revue canadienne de sante publique 65:29-33

35. Gunn JS, Stein DC. 1996. Use of a non-selective transformation technique to construct a multiply restriction/modification-deficient mutant of Neisseria gonorrhoeae. Molecular \& General Genetics : MGG 251:509-17

36. Hagman KE, Shafer WM. 1995. Transcriptional control of the mtr efflux system of Neisseria gonorrhoeae. Journal of Bacteriology 177:4162-5 
37. Han J, Wang Y, Sahin O, Shen Z, Guo B, et al. 2012. A fluoroquinolone resistance associated mutation in gyrA Affects DNA supercoiling in Campylobacter jejuni. Frontiers in Cellular and Infection Microbiology 2:21

38. Hauck C. 2003. 'Small' talk: Opa proteins as mediators of Neisseria-host-cell communication. Current Opinion in Microbiology 6:43-9

39. Jerse AE. 1999. Experimental gonococcal genital tract infection and opacity protein expression in estradiol-treated mice. Infection and Immunity 67:5699-708

40. Jerse AE, Bash MC, Russell MW. 2014. Vaccines against gonorrhea: current status and future challenges. Vaccine 32:1579-87

41. Jerse AE, Wu H, Packiam M, Vonck RA, Begum AA, Garvin LE. 2011. Estradiol-treated female mice as surrogate hosts for Neisseria gonorrhoeae genital tract infections. Frontiers in Microbiology 2:107-19

42. Jin W, Ibeagha-Awemu EM, Liang G, Beaudoin F, Zhao X, Guan le L. 2014. Transcriptome microRNA profiling of bovine mammary epithelial cells challenged with Escherichia coli or Staphylococcus aureus bacteria reveals pathogen directed microRNA expression profiles. BMC Genomics 15:181

43. Kampmeier RH. 1983. Introduction of sulfonamide therapy for gonorrhea. Sexually Transmitted Diseases 10:81-4

44. Kilmarx PH, Knapp JS, Xia M, St Louis ME, Neal SW, et al. 1998. Intercity spread of gonococci with decreased susceptibility to fluoroquinolones: a unique focus in the United States. The Journal of Infectious Diseases 177:677-82

45. Kirkcaldy RD, Kidd S, Weinstock HS, Papp JR, Bolan GA. 2013. Trends in antimicrobial resistance in Neisseria gonorrhoeae in the USA: the gonococcal isolate surveillance project (GISP), January 2006-June 2012. Sexually transmitted Infections 89 Suppl 4:iv5-iv10

46. Kugelberg E, Lofmark S, Wretlind B, Andersson DI. 2005. Reduction of the fitness burden of quinolone resistance in Pseudomonas aeruginosa. The Journal of Antimicrobial Chemotherapy 55:22-30

47. Kunz AN, Begum AA, Wu H, D'Ambrozio JA, Robinson JM, et al. 2012. Impact of fluoroquinolone resistance mutations on gonococcal fitness and in vivo selection for compensatory mutations. The Journal of Infectious Diseases 205:1821-9

48. Lankford CE, Skaggs PK. 1946. Cocarboxylase as a growth factor for certain strains of Neisseria gonorrhoeae. Archives of Biochemistry 9:265-83 
49. Lewis DA. 2013. The role of core groups in the emergence and dissemination of antimicrobial-resistant $N$. gonorrhoeae. Sexually Transmitted Infections 89 Suppl 4:iv47-iv51

50. Lewis LA, Choudhury B, Balthazar JT, Martin LE, Ram S, et al. 2009. Phosphoethanolamine substitution of lipid A and resistance of Neisseria gonorrhoeae to cationic antimicrobial peptides and complement-mediated killing by normal human serum. Infection and Immunity 77:1112-20

51. Luo N, Pereira S, Sahin O, Lin J, Huang S, et al. 2005. Enhanced in vivo fitness of fluoroquinolone-resistant Campylobacter jejuni in the absence of antibiotic selection pressure. Proceedings of the National Academy of Sciences of the United States of America 102:541-6

52. Maness MJ, Foster GC, Sparling PF. 1974. Ribosomal resistance to streptomycin and spectinomycin in Neisseria gonorrhoeae. Journal of bacteriology 120:1293-9

53. McChesney D, Tramont EC, Boslego JW, Ciak J, Sadoff J, Brinton CC. 1982. Genital antibody response to a parenteral gonococcal pilus vaccine. Infection and Immunity 36:1006-12

54. Merz AJ, So M. 2000. Interactions of pathogenic Neisseriae with epithelial cell membranes. Annual Review of Cell and Developmental Biology 16:423-57

55. Moran JS, Zenilman JM. 1990. Therapy for gonococcal infections: options in 1989. Reviews of Infectious Diseases 12 Suppl 6:S633-44

56. Morris SR, Moore DF, Hannah PB, Wang SA, Wolfe J, et al. 2009. Strain typing and antimicrobial resistance of fluoroquinolone-resistant Neisseria gonorrhoeae causing a California infection outbreak. Journal of Clinical Microbiology 47:2944-9

57. Ng LK, Martin I, Liu G, Bryden L. 2002. Mutation in 23S rRNA associated with macrolide resistance in Neisseria gonorrhoeae. Antimicrobial Agents and Chemotherapy 46:3020-5

58. O'Regan E, Quinn T, Frye JG, Pages JM, Porwollik S, et al. 2010. Fitness costs and stability of a high-level ciprofloxacin resistance phenotype in Salmonella enterica serotype enteritidis: reduced infectivity associated with decreased expression of Salmonella pathogenicity island 1 genes. Antimicrobial Agents and Chemotherapy 54:367-74

59. Ohneck E.A. DAJA, Kunz A.N., Jerse A.E., and Shafer W.M. 2012. Clinically Relevant Antibiotic Resistance Mechanisms Can Enhance the In Vivo Fitness of Neisseria gonorrhoeae. In Antibiotic Resistant Bacteria - A Continuous Challenge in the New Millennium: Intech. 
60. Ohneck EA, Zalucki YM, Johnson PJ, Dhulipala V, Golparian D, et al. 2011. A novel mechanism of high-level, broad-spectrum antibiotic resistance caused by a single base pair change in Neisseria gonorrhoeae. mBio 2

61. Ohnishi M, Golparian D, Shimuta K, Saika T, Hoshina S, et al. 2011. Is Neisseria gonorrhoeae initiating a future era of untreatable gonorrhea?: detailed characterization of the first strain with high-level resistance to ceftriaxone. Antimicrobial Agents and Chemotherapy 55:3538-45

62. Opel ML, Arfin SM, Hatfield GW. 2001. The effects of DNA supercoiling on the expression of operons of the ilv regulon of Escherichia coli suggest a physiological rationale for divergently transcribed operons. Molecular Microbiology 39:1109-15

63. Packiam M, Yedery RD, Begum AA, Carlson RW, Ganguly J, et al. 2014. Phosphoethanolamine decoration of Neisseria gonorrhoeae lipid A plays a dual immunostimulatory and protective role during experimental genital tract infection. Infection and Immunity 82:2170-9

64. Piddock LJ. 1999. Mechanisms of fluoroquinolone resistance: an update 19941998. Drugs 58 Suppl 2:11-8

65. Piek S, Ganguly, J., Anandan, A., Wanty, C., Stubbs, K., Scanlon, A., Vrielink, C.R., Kahler, C. 2012. Resistance to cationic antimicrobial peptides is determined by oxidoreductases in Neisseria sp. In Eighteenth International Pathogenic Neisseria Conference. Würzburg, Germany

66. Piek S, Wang Z, Ganguly J, Lakey AM, Bartley SN, et al. 2014. The role of oxidoreductases in determining the function of the neisserial lipid a phosphoethanolamine transferase required for resistance to polymyxin. PLoS One 9:e106513

67. Reich M, Liefeld T, Gould J, Lerner J, Tamayo P, Mesirov JP. 2006. GenePattern 2.0. Nature Genetics 38:500-1

68. Rouquette-Loughlin C, Dunham SA, Kuhn M, Balthazar JT, Shafer WM. 2003. The NorM Efflux Pump of Neisseria gonorrhoeae and Neisseria meningitidis Recognizes Antimicrobial Cationic Compounds. Journal of Bacteriology 185:1101-6

69. Rovinskiy N, Agbleke AA, Chesnokova O, Pang Z, Higgins NP. 2012. Rates of gyrase supercoiling and transcription elongation control supercoil density in a bacterial chromosome. PLoS Genetics 8:e1002845

70. Schulz zur Wiesch P, Engelstadter J, Bonhoeffer S. 2010. Compensation of fitness costs and reversibility of antibiotic resistance mutations. Antimicrobial Agents and Chemotherapy 54:2085-95 
71. Seib KL, Tseng HJ, McEwan AG, Apicella MA, Jennings MP. 2004. Defenses against oxidative stress in Neisseria gonorrhoeae and Neisseria meningitidis: distinctive systems for different lifestyles. The Journal of Infectious Diseases 190:136-47

72. Seib KL, Wu HJ, Kidd SP, Apicella MA, Jennings MP, McEwan AG. 2006. Defenses against oxidative stress in Neisseria gonorrhoeae: a system tailored for a challenging environment. Microbiology and Molecular Biology Reviews : MMBR 70:344-61

73. Sethi S, Sharma D, Mehta SD, Singh B, Smriti M, et al. 2006. Emergence of ciprofloxacin resistant Neisseria gonorrhoeae in north India. The Indian Journal of Medical Research 123:707-10

74. Shafer WM, Datta A, Kolli VS, Rahman MM, Balthazar JT, et al. 2002. Phase variable changes in genes $\lg t A$ and $\lg t C$ within the $\lg t A B C D E$ operon of Neisseria gonorrhoeae can modulate gonococcal susceptibility to normal human serum. Journal of Endotoxin research 8:47-58

75. Shafer WM, Qu X, Waring AJ, Lehrer RI. 1998. Modulation of Neisseria gonorrhoeae susceptibility to vertebrate antibacterial peptides due to a member of the resistance/nodulation/division efflux pump family. Proceedings of the National Academy of Sciences of the United States of America 95:1829-33

76. Siegel M, Olsen D, Critchlow C, Buchanan TM. 1982. Gonococcal pili: safety and immunogenicity in humans and antibody function in vitro. The Journal of Infectious Diseases 145:300-10

77. Sissi C, Palumbo M. 2010. In front of and behind the replication fork: bacterial type IIA topoisomerases. Cellular and Molecular Life Sciences : CMLS 67:200124

78. Slaney L, Chubb H, Ronald A, Brunham R. 1990. In-vitro activity of azithromycin, erythromycin, ciprofloxacin and norfloxacin against Neisseria gonorrhoeae, Haemophilus ducreyi, and Chlamydia trachomatis. The Journal of Antimicrobial Chemotherapy 25 Suppl A:1-5

79. Soler-Garcia AA, Jerse AE. 2004. A Neisseria gonorrhoeae catalase mutant is more sensitive to hydrogen peroxide and paraquat, an inducer of toxic oxygen radicals. Microbial Pathogenesis 37:55-63

80. Song W, Condron S, Mocca BT, Veit SJ, Hill D, et al. 2008. Local and humoral immune responses against primary and repeat Neisseria gonorrhoeae genital tract infections of 17beta-estradiol-treated mice. Vaccine 26:5741-51

81. Sparling PF. 1999. Biology of Neisseria gonorrhoeae. pp 433-449. McGraw-Hill 
82. Stohl EA, Criss AK, Seifert HS. 2005. The transcriptome response of Neisseria gonorrhoeae to hydrogen peroxide reveals genes with previously uncharacterized roles in oxidative damage protection. Molecular Microbiology 58:520-32

83. Su X, Lind I. 2001. Molecular basis of high-level ciprofloxacin resistance in Neisseria gonorrhoeae strains isolated in Denmark from 1995 to 1998.

Antimicrobial Agents and Chemotherapy 45:117-23

84. Szafranska AK, Oxley AP, Chaves-Moreno D, Horst SA, Rosslenbroich S, et al. 2014. High-Resolution Transcriptomic Analysis of the Adaptive Response of Staphylococcus aureus during Acute and Chronic Phases of Osteomyelitis. mBio 5

85. Tanaka M, Nakayama H, Haraoka M, Saika T. 2000. Antimicrobial resistance of Neisseria gonorrhoeae and high prevalence of ciprofloxacin-resistant isolates in Japan, 1993 to 1998. Journal of Clinical Microbiology 38:521-5

86. Taylor-Robinson D, Furr PM, Hetherington CM. 1990. Neisseria gonorrhoeae colonises the genital tract of oestradiol-treated germ-free female mice. Microbial Pathogenesis 9:369-73

87. Thornsberry C, Jaffee H, Brown ST, Edwards T, Biddle JW, Thompson SE. 1977. Spectinomycin-resistant Neisseria gonorrhoeae. JAMA : Journal of the American Medical Association 237:2405-6

88. Tong Y, Arking D, Ye S, Reinhold B, Reinhold V, Stein DC. 2002. Neisseria gonorrhoeae strain PID2 simultaneously expresses six chemically related lipooligosaccharide structures. Glycobiology 12:523-33

89. Tramont EC, Sadoff JC, Boslego JW, Ciak J, McChesney D, et al. 1981. Gonococcal pilus vaccine. Studies of antigenicity and inhibition of attachment. The Journal of Clinical Investigation 68:881-8

90. Travers A, Muskhelishvili G. 2005. DNA supercoiling - a global transcriptional regulator for enterobacterial growth? Nature Reviews. Microbiology 3:157-69

91. Trees DL SA, Neal SW, Higa H, Knapp JS. Molecular epidemiology of Neisseria gonorrhoeae exhibiting decreased susceptibility and resistance to ciprofloxacin in Hawaii, 1991-1999. Sexually Transmitted Diseases

92. Trees DL, Sandul AL, Neal SW, Higa H, Knapp JS. 2001. Molecular epidemiology of Neisseria gonorrhoeae exhibiting decreased susceptibility and resistance to ciprofloxacin in Hawaii, 1991-1999. Sexually Transmitted Diseases 28:309-14

93. Trees DL, Sirivongrangson P, Schultz AJ, Buatiang A, Neal SW, et al. 2002. Multiclonal increase in ciprofloxacin-resistant Neisseria gonorrhoeae, Thailand, 1998-1999. Sexually Transmitted Diseases 29:668-73 
94. Unemo M, Golparian D, Skogen V, Olsen AO, Moi H, et al. 2013. Neisseria gonorrhoeae strain with high-level resistance to spectinomycin due to a novel resistance mechanism (mutated ribosomal protein S5) verified in Norway. Antimicrobial Agents and Chemotherapy 57:1057-61

95. Unemo M, Shafer WM. 2011. Antibiotic resistance in Neisseria gonorrhoeae: origin, evolution, and lessons learned for the future. Annals of the New York Academy of Sciences 1230:E19-28

96. Unemo M, Shafer WM. 2014. Antimicrobial resistance in Neisseria gonorrhoeae in the 21st century: past, evolution, and future. Clinical Microbiology Reviews 27:587-613

97. Warner DM, Folster JP, Shafer WM, Jerse AE. 2007. Regulation of the MtrCMtrD-MtrE efflux-pump system modulates the in vivo fitness of Neisseria gonorrhoeae. The Journal of Infectious Diseases 196:1804-12

98. Warner DM, Shafer WM, Jerse AE. 2008. Clinically relevant mutations that cause derepression of the Neisseria gonorrhoeae MtrC-MtrD-MtrE Efflux pump system confer different levels of antimicrobial resistance and in vivo fitness. Molecular Microbiology 70:462-78

99. Warrier I, Hicks LD, Battisti JM, Raghavan R, Minnick MF. 2014. Identification of novel small RNAs and characterization of the 6S RNA of Coxiella burnetii. PLoS One 9:e100147

100. Webber MA, Ricci V, Whitehead R, Patel M, Fookes M, et al. 2013. Clinically relevant mutant DNA gyrase alters supercoiling, changes the transcriptome, and confers multidrug resistance. mBio 4

101. Williams NL, Howells AJ, Maxwell A. 2001. Locking the ATP-operated clamp of DNA gyrase: probing the mechanism of strand passage. Journal of Molecular Biology 306:969-84

102. Wu H, Jerse AE. 2006. Alpha-2,3-sialyltransferase enhances Neisseria gonorrhoeae survival during experimental murine genital tract infection. Infection and Immunity 74:4094-103

103. Wu H, Soler-Garcia AA, Jerse AE. 2009. A strain-specific catalase mutation and mutation of the metal-binding transporter gene mntC attenuate Neisseria gonorrhoeae in vivo but not by increasing susceptibility to oxidative killing by phagocytes. Infection and Immunity 77:1091-102

104. Xiong X, Bromley EH, Oelschlaeger P, Woolfson DN, Spencer J. 2011.

Structural insights into quinolone antibiotic resistance mediated by pentapeptide repeat proteins: conserved surface loops direct the activity of a Qnr protein from a gram-negative bacterium. Nucleic Acids Research 39:3917-27 
105. Yang QL, Gotschlich EC. 1996. Variation of gonococcal lipooligosaccharide structure is due to alterations in poly-G tracts in lgt genes encoding glycosyl transferases. The Journal of Experimental Medicine 183:323-7

106. Zhao LH, Zhao SP. 2013. Molecular basis of high-level ciprofloxacin resistance in Neisseria gonorrhoeae strains from Shandong Province, China. Brazilian Journal of Microbiology : [publication of the Brazilian Society for Microbiology] 44:273-6 